\title{
SECOND-ORDER ANALYSIS OF AN OPTIMAL CONTROL PROBLEM IN A PHASE FIELD TUMOR GROWTH MODEL WITH SINGULAR POTENTIALS AND CHEMOTAXIS
}

\author{
Pierluigi Colli ${ }^{1, *} \oplus$, Andrea Signori ${ }^{1}$ And Jürgen Sprekels ${ }^{2,3}$
}

\begin{abstract}
This paper concerns a distributed optimal control problem for a tumor growth model of Cahn-Hilliard type including chemotaxis with possibly singular potentials, where the control and state variables are nonlinearly coupled. First, we discuss the weak well-posedness of the system under very general assumptions for the potentials, which may be singular and nonsmooth. Then, we establish the strong well-posedness of the system in a reduced setting, which however admits the logarithmic potential: this analysis will lay the foundation for the study of the corresponding optimal control problem. Concerning the optimization problem, we address the existence of minimizers and establish both first-order necessary and second-order sufficient conditions for optimality. The mathematically challenging second-order analysis is completely performed here, after showing that the solution mapping is twice continuously differentiable between suitable Banach spaces via the implicit function theorem. Then, we completely identify the second-order Fréchet derivative of the control-to-state operator and carry out a thorough and detailed investigation about the related properties.
\end{abstract}

Mathematics Subject Classification. 49J20, 49K20, 49K40, 35K57, 37N25.

Received September 16, 2020. Accepted June 24, 2021.

\section{INTRODUCTION}

Lots of disclosures have been obtained in the past decades concerning tumor growth modeling: see, e.g., the pioneering works $[13,14,48]$. The main advantage of a mathematical approach is to be capable of predicting and analyzing tumor growth behavior without inflicting any harm to the patients, thus helping medical practitioners to plan the clinical medications.

The phase field approach to tumor modeling consists in describing the tumor fraction by means of an order parameter $\varphi$ representing the concentration of the tumor, which usually is normalized to range between -1 and 1. Namely, the level sets $\{\varphi=1\}$ and $\{\varphi=-1\}$ may describe the regions of pure phases: the tumorous phase and the healthy phase, respectively. Moreover, the diffuse interface approach postulates the existence of a thin transition layer $\{-1<\varphi<1\}$ in which the phase variable passes rapidly, but continuously, from one phase to the other. We assume the growth and proliferation of the tumor to be driven by the absorption and consumption of

Keywords and phrases: Optimal control, tumor growth models, singular potentials, optimality conditions, second-order analysis.

1 Dipartimento di Matematica "F. Casorati", Università di Pavia, via Ferrata 5, 27100 Pavia, Italy.

2 Department of Mathematics, Humboldt-Universität zu Berlin, Unter den Linden 6, 10099 Berlin, Germany.

3 Weierstrass Institute for Applied Analysis and Stochastics, Mohrenstrasse 39, 10117 Berlin, Germany.

* Corresponding author: pierluigi.colli@unipv.it 
some nutrient, so that the equation for the phase variable, which has a Cahn-Hilliard type structure, is coupled with a reaction-diffusion equation for the variable $\sigma$ capturing the evolution of an unknown species nutrient (e.g., oxygen, glucose) in which the tissue under consideration is embedded.

Let $\alpha>0, \beta>0$, and let $\Omega \subset \mathbb{R}^{3}$ denote some open and bounded domain having a smooth boundary $\Gamma=\partial \Omega$ ( $\Omega$ of class $C^{2}$ would be sufficient). We indicate by $\mathbf{n}$ the unit outward normal on $\Gamma$ with corresponding outward normal derivative $\partial_{\mathbf{n}}$. Moreover, we fix some final time $T>0$ and introduce for every $t \in(0, T]$ the sets $Q_{t}:=\Omega \times(0, t), Q_{t}^{T}:=\Omega \times(t, T)$, and $\Sigma_{t}:=\Gamma \times(0, t)$, where we put, for the sake of brevity, $Q:=Q_{T}$ and $\Sigma:=\Sigma_{T}$. We then consider the following optimal control problem:

Minimize the cost functional

$$
\mathcal{J}((\mu, \varphi, \sigma), \mathbf{u}):=\frac{b_{1}}{2} \int_{Q}\left|\varphi-\widehat{\varphi}_{Q}\right|^{2}+\frac{b_{2}}{2} \int_{\Omega}\left|\varphi(T)-\widehat{\varphi}_{\Omega}\right|^{2}+\frac{b_{0}}{2} \int_{Q}|\mathbf{u}|^{2}
$$

subject to the state system

$$
\begin{array}{lc}
\alpha \partial_{t} \mu+\partial_{t} \varphi-\Delta \mu=P(\varphi)(\sigma+\chi(1-\varphi)-\mu)-\mathbb{h}(\varphi) u_{1} & \text { in } Q, \\
\beta \partial_{t} \varphi-\Delta \varphi+F^{\prime}(\varphi)=\mu+\chi \sigma & \text { in } Q, \\
\partial_{t} \sigma-\Delta \sigma=-\chi \Delta \varphi-P(\varphi)(\sigma+\chi(1-\varphi)-\mu)+u_{2} & \text { in } Q, \\
\partial_{\mathbf{n}} \mu=\partial_{\mathbf{n}} \varphi=\partial_{\mathbf{n}} \sigma=0 & \text { on } \Sigma, \\
\mu(0)=\mu_{0}, \quad \varphi(0)=\varphi_{0}, \quad \sigma(0)=\sigma_{0}, & \text { in } \Omega,
\end{array}
$$

and to the control constraint

$$
\mathbf{u}=\left(u_{1}, u_{2}\right) \in \mathcal{U}_{\mathrm{ad}} .
$$

Here, the constants $b_{1}, b_{2}$ are nonnegative, while $b_{0}$ is positive. Moreover, $\widehat{\varphi}_{Q}$ and $\widehat{\varphi}_{\Omega}$ are given target functions, and the set of admissible controls $\mathfrak{U}_{\mathrm{ad}}$ is a nonempty, closed and convex subset of the control space

$$
\mathcal{U}:=L^{\infty}(Q)^{2} .
$$

The state system (1.2)-(1.6) constitutes a simplified and relaxed version of the four-species thermodynamically consistent model for tumor growth originally proposed by Hawkins-Daruud et al. in [33] that additionally includes chemotaxis effects. Let us briefly review the role of the occurring symbols. The primary variables $\varphi, \mu$ and $\sigma$ denote the phase field, the associated chemical potential, and the nutrient concentration, respectively. Furthermore, we stress that the additional term $\alpha \partial_{t} \mu$ corresponds to a parabolic regularization for equation (1.2), whereas the term $\beta \partial_{t} \varphi$ is the viscosity contribution to the Cahn-Hilliard equation. The key idea behind these regularizations originates from the fact that their presence allows us to take into account more general potentials that may be singular and possibly nonregular. The nonlinearity $P$ denotes a proliferation function, whereas the positive constant $\chi$ represents the chemotactic sensitivity. Lastly, as a common feature of phase field models, $F$ is a nonlinearity which is assumed to possess a double-well shape. Typical examples are given by the regular, logarithmic, and double obstacle potentials, which are defined, in this order, by

$$
\begin{aligned}
& F_{\text {reg }}(r)=\frac{1}{4}\left(1-r^{2}\right)^{2} \quad \text { for } r \in \mathbb{R}, \\
& F_{\log }(r)= \begin{cases}(1+r) \ln (1+r)+(1-r) \ln (1-r)-k_{1} r^{2} & \text { for } r \in(-1,1) \\
2 \ln (2)-k_{1} & \text { for } r \in\{-1,1\}, \\
+\infty & \text { for } r \notin[-1,1]\end{cases}
\end{aligned}
$$




$$
F_{\text {obs }}(r)=\left\{\begin{array}{ll}
k_{2}\left(1-r^{2}\right) & \text { for } r \in[-1,1] \\
+\infty & \text { for } r \notin[-1,1]
\end{array},\right.
$$

where $k_{1}>1$ and $k_{2}>0$ so that $F_{\log }$ and $F_{\text {obs }}$ are nonconvex. Observe that $F_{\log }$ is very relevant in the applications, where $F_{\log }^{\prime}(r)$ becomes unbounded as $r \searrow-1$ and $r \nearrow+1$, and that in the case of (1.11) the second equation (1.3) has to be interpreted as a differential inclusion, where $F^{\prime}(\varphi)$ is understood in the sense of subdifferentials.

In this paper, we take two distributed controls that act in the phase equation and in the nutrient equation, respectively. The control variable $u_{1}$, which is nonlinearly coupled to the state variable $\varphi$ in the phase equation (1.2), models the application of a cytotoxic drug into the system; it is multiplied by a truncation function $\mathrm{h}(\cdot)$ in order to have the action only in the spatial region where the tumor cells are located. For instance, it can be assumed that $\mathfrak{h}(-1)=0, \mathfrak{h}(1)=1, \mathfrak{h}(\varphi)$ is in between if $-1<\varphi<1$; see [24,30,34,35] for some insights on possible choices of $\mathfrak{h}$. On the other hand, the control $u_{2}$ can model either an external medication or some nutrient supply.

As far as well-posedness is concerned, the above model has already been investigated in the case $\chi=0$ in [5, 7-9], and in [20] with $\alpha=\beta=\chi=0$. There the authors also pointed out how $\alpha$ and $\beta$ can be set to zero, by providing the proper framework in which a limit system can be identified and uniquely solved. We also note that in [11] a version has been studied in which the Laplacian in the equations (1.2)-(1.4) has been replaced by fractional powers of a more general class of selfadjoint operators having compact resolvents.

For some nonlocal variations of the above model we refer to $[22,23,38]$. Moreover, in order to better emulate in-vivo tumor growth, it is possible to include in similar models the effects generated by the fluid flow development by postulating a Darcy's law or a Stokes-Brinkman's law. In this direction, we refer to $[15,19,22,24-28,30,48]$, and we also mention [31], where elastic effects are included. For further models, discussing the case of multispecies, we address the reader to [15, 22].

The investigation of the associated optimal control problem also presents a wide number of results of which we mention [10-12, 17, 18, 23, 29, 35, 39-43, 45, 46]. Notice that, despite the number of contributions, only [17] established second-order optimality conditions under suitable restrictions on the considered model. In particular, the authors of [17] avoid considering the chemotaxis effects and allow only regular potentials to be considered.

In this paper, first we discuss the weak well-posedness of the system (1.2)-(1.6) in a very general framework for the potentials, which includes all of the cases in (1.9)-(1.11). Then, we turn our attention to the strong well-posedness of (1.2)-(1.6) in the cases of the regular $F_{\text {reg }}$ and logarithmic $F_{\text {log }}$ potentials. This is done in Section 2, while the corresponding optimal control problem is investigated in the following sections. Section 3 is concerned with the existence of minimizers, then the intensive and crucial Section 4 establishes the differentiability properties of the control-to-state operator and contains a number of results on the concerned linearized problems and the basic stability estimates for the solutions. The last two Sections 5 and 6 treat in some detail the first-order necessary and second-order sufficient conditions for optimality, respectively. Let us point out that the second-order analysis is challenging from the mathematical viewpoint and demands to prove that the solution mapping is twice continuously differentiable between suitable Banach spaces. By taking advantage of the regularizing effect due to the aforementioned relaxation terms, we can deal with a complete study of the secondorder analysis, still covering the case of singular potentials and chemotaxis. Moreover, we are able to identify the second-order Fréchet derivative of the control-to-state operator and investigate the related properties in a sharp and profound way.

Throughout the paper, we make repeated use of Hölder's inequality, of the elementary Young's inequality

$$
a b \leq \delta|a|^{2}+\frac{1}{4 \delta}|b|^{2} \quad \forall a, b \in \mathbb{R}, \quad \forall \delta>0,
$$

as well as the continuity of the embeddings $H^{1}(\Omega) \subset L^{p}(\Omega)$ for $1 \leq p \leq 6$ and $H^{2}(\Omega) \subset C^{0}(\bar{\Omega})$. Notice that the latter embedding is also compact, while this holds true for the former embeddings only if $p<6$. 
Lastly, let us introduce a convention that will be tacitly employed in the rest of the paper: the symbol smallcase $c$ is used to indicate every constant that depends only on the structural data of the problem (such as $T$, $\Omega, \alpha$ or $\beta$, the shape of the nonlinearities, and the norms of the involved functions), so that its meaning may change from line to line. When a parameter $\delta$ enters the computation, then the symbol $c_{\delta}$ denotes constants that depend on $\delta$ in addition. On the contrary, precise constants we could refer to are treated in a different way.

\section{General Setting And PRoperties of the State System}

In this section, we introduce the general setting of our control problem and state some results on the state system (1.2)-(1.6). To begin with, for a Banach space $X$ we denote by $\|\cdot\|_{X}$ the norm in the space $X$ or in a power thereof, and by $X^{*}$ its dual space. The only exeption from this rule applies to the norms of the $L^{p}$ spaces and of their powers, which we often denote by $\|\cdot\|_{p}$, for $1 \leq p \leq \infty$. As usual, for Banach spaces $X$ and $Y$ we introduce the linear space $X \cap Y$ which becomes a Banach space when equipped with its natural norm $\|u\|_{X \cap Y}:=\|u\|_{X}+\|u\|_{Y}$, for $u \in X \cap Y$. Moreover, we recall the definition (1.8) of $\mathcal{U}$ and introduce the spaces

$$
H:=L^{2}(\Omega), \quad V:=H^{1}(\Omega), \quad W_{0}:=\left\{v \in H^{2}(\Omega): \partial_{\mathbf{n}} v=0 \text { on } \Gamma\right\} .
$$

Furthermore, by $(\cdot, \cdot),\|\cdot\|$, and $\langle\cdot, \cdot\rangle$, we denote the standard inner product and related norm in $H$, as well as the dual product between $V$ and its dual $V^{*}$. For given final time $T>0$, we introduce the spaces

$$
\begin{aligned}
& Z:=H^{1}(0, T ; H) \cap L^{\infty}(0, T ; V) \cap L^{2}\left(0, T ; W_{0}\right), \quad z:=Z \times Z \times Z, \\
& \mathcal{V}:=\left(L^{\infty}(0, T ; H) \cap L^{2}(0, T ; V)\right) \times Z \times\left(L^{\infty}(0, T ; H) \cap L^{2}(0, T ; V)\right),
\end{aligned}
$$

which are Banach spaces when endowed with their natural norms.

Some assumptions on the data are stated here.

(W1) $\alpha, \beta$ and $\chi$ are positive constants.

(W2) $F=F_{1}+F_{2}$, where $F_{1}: \mathbb{R} \rightarrow[0,+\infty]$ is convex and lower semicontinuous with $F_{1}(0)=0$, and where $F_{2} \in C^{1}(\mathbb{R})$ has a Lipschitz continuous derivative $F_{2}^{\prime}$.

(W3) $P \in C^{0}(\mathbb{R})$ is nonnegative, bounded, and Lipschitz continuous.

(W4) $\mathbb{h} \in C^{0}(\mathbb{R})$ is nonnegative, bounded, and Lipschitz continuous.

For the sake of simplicity, we indicate with a common notation

$L$ as a Lipschitz constant for $F_{2}^{\prime}, P$, and $\mathbb{h}$.

Let us note that all of the choices (1.9)-(1.11) are admitted for the potentials. In fact, the assumption (W2) implies that the subdifferential $\partial F_{1}$ of $F_{1}$ is a maximal monotone graph in $\mathbb{R} \times \mathbb{R}$ with effective domain $D\left(\partial F_{1}\right) \subset$ $D\left(F_{1}\right)$, and, since $F_{1}$ attains the minimum value 0 at 0 , it turns out that $0 \in D\left(\partial F_{1}\right)$ and $0 \in \partial F_{1}(0)$. Now, in the general setting depicted by $(\mathbf{W} 1)-(\mathbf{W} 4)$, we are able to provide a first well-posedness result for the system (1.2)-(1.6). First, let us present the notion of weak solution to (1.2)-(1.6).

Definition 2.1. A quadruplet $(\mu, \varphi, \xi, \sigma)$ is called a weak solution to the initial boundary value problem $(1.2)-(1.6)$ if

$$
\begin{aligned}
\varphi & \in H^{1}(0, T ; H) \cap L^{\infty}(0, T ; V) \cap L^{2}\left(0, T ; W_{0}\right), \\
\mu, \sigma & \in H^{1}\left(0, T ; V^{*}\right) \cap L^{\infty}(0, T ; H) \cap L^{2}(0, T ; V), \\
\xi & \in L^{2}(0, T ; H),
\end{aligned}
$$


and if $(\mu, \varphi, \xi, \sigma)$ satisfies the corresponding weak formulation given by

$$
\begin{aligned}
& \left\langle\partial_{t}(\alpha \mu+\varphi), v\right\rangle+\int_{\Omega} \nabla \mu \cdot \nabla v=\int_{\Omega} P(\varphi)(\sigma+\chi(1-\varphi)-\mu) v-\int_{\Omega} \mathbb{h}(\varphi) u_{1} v \\
& \quad \text { for every } v \in V \text { and a.e. in }(0, T), \\
& \beta \partial_{t} \varphi-\Delta \varphi+\xi+F_{2}^{\prime}(\varphi)=\mu+\chi \sigma, \quad \xi \in \partial F_{1}(\varphi), \text { a.e. in } Q, \\
& \left\langle\partial_{t} \sigma, v\right\rangle+\int_{\Omega} \nabla \sigma \cdot \nabla v=\chi \int_{\Omega} \nabla \varphi \cdot \nabla v-\int_{\Omega} P(\varphi)(\sigma+\chi(1-\varphi)-\mu) v+\int_{\Omega} u_{2} v \\
& \quad \text { for every } v \in V \text { and a.e. in }(0, T),
\end{aligned}
$$

as well as

$$
\mu(0)=\mu_{0}, \quad \varphi(0)=\varphi_{0}, \quad \sigma(0)=\sigma_{0}, \quad \text { a.e. in } \Omega .
$$

It is worth noticing that the homogeneous Neumann boundary conditions (1.5) are considered in the condition (2.5) for $\varphi\left(c f\right.$. the definition of the space $\left.W_{0}\right)$ and incorporated in the variational equalities $(2.8)$ and $(2.10)$ for $\mu$ and $\sigma$, when using the forms $\int_{\Omega} \nabla \mu \cdot \nabla v$ and $\int_{\Omega} \nabla \sigma \cdot \nabla v$. Moreover, let us point out that, at this level, the control pair $\left(u_{1}, u_{2}\right)$ just yields two fixed forcing terms in (2.8) and (2.10). The initial conditions (2.11) make sense since (2.5) and (2.6) ensure that $\varphi$ and $\mu, \sigma$ are continuous from $[0, T]$ to $V$ and $H$, respectively.

Theorem 2.2 (Weak well-posedness). Assume that (W1)-(W4) hold. Moreover, let the initial data $\left(\mu_{0}, \varphi_{0}, \sigma_{0}\right)$ satisfy

$$
\mu_{0}, \sigma_{0} \in L^{2}(\Omega), \quad \varphi_{0} \in H^{1}(\Omega), \quad F_{1}\left(\varphi_{0}\right) \in L^{1}(\Omega),
$$

and suppose that the source terms $u_{1}, u_{2}$ are such that

$$
\left(u_{1}, u_{2}\right) \in L^{2}(Q) \times L^{2}(Q) .
$$

Then there exists at least one solution $(\mu, \varphi, \xi, \sigma)$ in the sense of Definition 2.1. Moreover, if $u_{1} \in L^{\infty}(Q)$ in addition to (2.13), then the found solution is unique. Furthermore, let $\left(\mu_{i}, \varphi_{i}, \xi_{i}, \sigma_{i}\right), i=1,2$, be two weak solutions to (1.2)-(1.6) associated with the initial data $\left(\mu_{0}^{i}, \varphi_{0}^{i}, \sigma_{0}^{i}\right)$, which satisfy (2.12), and controls $\left(u_{1}^{i}, u_{2}^{i}\right) \in$ $L^{\infty}(Q) \times L^{2}(Q), i=1,2$. Then there is a positive constant $C_{d}$, depending only on the data of the system, such that

$$
\begin{aligned}
& \left\|\alpha\left(\mu_{1}-\mu_{2}\right)+\left(\varphi_{1}-\varphi_{2}\right)\right\|_{L^{\infty}(0, T ; H)}+\left\|\nabla\left(\mu_{1}-\mu_{2}\right)\right\|_{L^{2}(0, T ; H)} \\
& \quad+\left\|\varphi_{1}-\varphi_{2}\right\|_{L^{\infty}(0, T ; H) \cap L^{2}(0, T ; V)}+\left\|\sigma_{1}-\sigma_{2}\right\|_{L^{\infty}(0, T ; H) \cap L^{2}(0, T ; V)} \\
& \leq C_{d}\left(\left\|\alpha\left(\mu_{0}^{1}-\mu_{0}^{2}\right)+\left(\varphi_{0}^{1}-\varphi_{0}^{2}\right)\right\|+\left\|\varphi_{0}^{1}-\varphi_{0}^{2}\right\|+\left\|\sigma_{0}^{1}-\sigma_{0}^{2}\right\|\right) \\
& \quad+C_{d}\left(\left\|u_{1}^{1}-u_{1}^{2}\right\|_{L^{2}(0, T ; H)}+\left\|u_{2}^{1}-u_{2}^{2}\right\|_{L^{2}(0, T ; H)}\right) .
\end{aligned}
$$

Before entering the proof, let us remark that the above result is very general and includes also the cases of singular and nonsmooth potentials, such as the double obstacle potential defined by (1.11). For the dependencies of the constant $C_{d}$, we invite the reader to follow the proof of the estimate (2.14) below.

Proof. For the proof of the existence of a solution, we point out that the arguments are quite standard, since similar procedures have already been used in previous contributions. Thus, for that part, we proceed rather formally, just employing the Yosida approximation of $\partial F_{1}$ for our estimates, without recurring to finite-dimensional approximation techniques like the Faedo-Galerkin scheme. 
Hence, we introduce the Yosida regularization of $\partial F_{1}$. For $\varepsilon>0$ let $F_{1, \varepsilon}$ denote the Moreau-Yosida approximation of $F_{1}$ at the level $\varepsilon$. It is well known (see, e.g., [2]) that the following conditions are satisfied:

$$
\begin{aligned}
& 0 \leq F_{1, \varepsilon}(r) \leq F_{1}(r) \text { for all } r \in \mathbb{R} \text {. } \\
& F_{1, \varepsilon}^{\prime} \text { is Lipschitz continuous on } \mathbb{R} \text { with Lipschitz constant } \frac{1}{\varepsilon} \text {, and } F_{1, \varepsilon}^{\prime}(0)=0 . \\
& \left|F_{1, \varepsilon}^{\prime}(r)\right| \leq\left|\left(\partial F_{1}\right)^{\circ}(r)\right| \text { and } \lim _{\varepsilon \searrow 0} F_{1, \varepsilon}^{\prime}(r)=\left(\partial F_{1}\right)^{\circ}(r), \quad \text { for all } r \in D\left(\partial F_{1}\right) .
\end{aligned}
$$

Here, $\left(\partial F_{1}\right)^{\circ}$ denotes the minimal section of $\partial F_{1}$, that is, $\left(\partial F_{1}\right)^{\circ}(r)$ defines the element of $\left(\partial F_{1}\right)(r)$ with minimal modulus.

Next, we are going to prove a series of estimates for the solution to problem $(2.8)-(2.10)$, where $\left(\partial F_{1}\right)(r)$ is replaced by $F_{1, \varepsilon}^{\prime}$ and the inclusion in (2.9) reduces to an equality. Namely, we argue on

$$
\beta \partial_{t} \varphi-\Delta \varphi+F_{1, \varepsilon}^{\prime}(\varphi)+F_{2}^{\prime}(\varphi)=\mu+\chi \sigma \quad \text { a.e. in } Q .
$$

For the sake of simplicity, we still denote by $(\mu, \varphi, \xi, \sigma)$, with $\xi=F_{1, \varepsilon}^{\prime}(\varphi)$, the solution to the approximated system in place of $\left(\mu_{\varepsilon}, \varphi_{\varepsilon}, \xi_{\varepsilon}, \sigma_{\varepsilon}\right)$; the correct notation will be reintroduced at the end of each estimate.

First Estimate: We add the term $\varphi$ to both sides of (2.18) and test by $\partial_{t} \varphi$. Then, we take $v=\mu$ in (2.8) and $v=\sigma$ in (2.10). Moreover, we add the resulting equalities and, with the help of a cancellation, we deduce that almost everywhere in $(0, T)$ it holds the identity

$$
\begin{aligned}
\frac{1}{2} \frac{\mathrm{d}}{\mathrm{d} t} & \left(\alpha\|\mu\|^{2}+\|\varphi\|_{V}^{2}+2 \int_{\Omega} F_{1, \varepsilon}(\varphi)+\|\sigma\|^{2}\right) \\
& +\|\nabla \mu\|^{2}+\beta\left\|\partial_{t} \varphi\right\|^{2}+\|\nabla \sigma\|^{2}+\int_{\Omega} P(\varphi)(\mu-\sigma)^{2} \\
= & \int_{\Omega} \chi P(\varphi)(1-\varphi)(\mu-\sigma)-\int_{\Omega} \mathbb{h}(\varphi) u_{1} \mu+\int_{\Omega} \chi \sigma \partial_{t} \varphi \\
& +\int_{\Omega}\left(\varphi-F_{2}^{\prime}(\varphi)\right) \partial_{t} \varphi+\chi \int_{\Omega} \nabla \varphi \cdot \nabla \sigma+\int_{\Omega} u_{2} \sigma=: I .
\end{aligned}
$$

Note that the last term on the left-hand side is nonnegative due to (W3). Then, we can integrate the above inequality over $[0, t]$ for $t \in(0, T]$, using the initial conditions $(2.11)$. We point out that the quantity

$$
\alpha\left\|\mu_{0}\right\|^{2}+\left\|\varphi_{0}\right\|_{V}^{2}+2 \int_{\Omega} F_{1, \varepsilon}\left(\varphi_{0}\right)+\left\|\sigma_{0}\right\|^{2} \quad \text { is bounded independently of } \varepsilon
$$

thanks to (2.12) and (2.15). Next, owing to the boundedness and regularity properties of $P, \mathfrak{h}$ and $F_{2}^{\prime}$, and by Young's inequality, it is straightforward to infer that

$$
\begin{aligned}
\int_{0}^{t} I(s) \mathrm{d} s \leq & \chi\|P\|_{\infty} \int_{Q_{t}}\left(1+|\varphi|^{2}+|\mu|^{2}+|\sigma|^{2}\right)+\frac{1}{2}\|\mathbb{h}\|_{\infty} \int_{Q_{t}}\left(\left|u_{1}\right|^{2}+|\mu|^{2}\right) \\
& +\frac{\beta}{4} \int_{Q_{t}}\left|\partial_{t} \varphi\right|^{2}+\frac{\chi^{2}}{\beta} \int_{Q_{t}}|\sigma|^{2}+\frac{\beta}{4} \int_{Q_{t}}\left|\partial_{t} \varphi\right|^{2}+c \int_{Q_{t}}\left(1+|\varphi|^{2}\right) \\
& +\frac{1}{2} \int_{Q_{t}}|\nabla \sigma|^{2}+\frac{\chi^{2}}{2} \int_{Q_{t}}|\nabla \varphi|^{2}+\frac{1}{2} \int_{Q_{t}}\left(\left|u_{2}\right|^{2}+|\sigma|^{2}\right) \\
\leq & \int_{0}^{t}\left(\frac{\beta}{2}\left\|\partial_{t} \varphi(s)\right\|^{2}+\frac{1}{2}\|\nabla \sigma(s)\|^{2}\right) \mathrm{d} s+c
\end{aligned}
$$




$$
+c \int_{0}^{t}\left(\|\varphi(s)\|_{V}^{2}+\|\mu(s)\|^{2}+\|\sigma(s)\|^{2}+\left\|u_{1}(s)\right\|^{2}+\left\|u_{2}(s)\right\|^{2}\right) \mathrm{d} s
$$

so that it suffices to apply Gronwall's lemma to conclude that

$$
\begin{aligned}
& \left\|\mu_{\varepsilon}\right\|_{L^{\infty}(0, T ; H) \cap L^{2}(0, T ; V)}+\left\|\varphi_{\varepsilon}\right\|_{H^{1}(0, T ; H) \cap L^{\infty}(0, T ; V)} \\
& \quad+\left\|F_{1, \varepsilon}\left(\varphi_{\varepsilon}\right)\right\|_{L^{\infty}\left(0, T ; L^{1}(\Omega)\right)}^{1 / 2}+\left\|\sigma_{\varepsilon}\right\|_{L^{\infty}(0, T ; H) \cap L^{2}(0, T ; V)} \leq c .
\end{aligned}
$$

SeCond Estimate: Now, owing to (2.19), from a comparison of terms in the variational equalities (2.8) and (2.10) it follows that

$$
\left\|\partial_{t} \mu_{\varepsilon}\right\|_{L^{2}\left(0, T ; V^{*}\right)}+\left\|\partial_{t} \sigma_{\varepsilon}\right\|_{L^{2}\left(0, T ; V^{*}\right)} \leq c .
$$

In fact, arguing for instance on (2.8), and taking advantage of (W3), (W4), (2.13) and (2.19), we have that for a.e. $t \in(0, T)$ and for every $v \in V$ it holds

$$
\begin{aligned}
\left|\left\langle\alpha \partial_{t} \mu(t), v\right\rangle\right| \leq & \left\|\partial_{t} \varphi(t)\right\|\|v\|+\|\nabla \mu(t)\|\|\nabla v\| \\
& +c(\|\sigma(t)\|+\|\varphi(t)\|+1+\|\mu(t)\|)\|v\|+c\left\|u_{1}(t)\right\|\|v\| \\
\leq & c\left(\left\|\partial_{t} \varphi(t)\right\|+\left\|u_{1}(t)\right\|+1\right)\|v\|_{V} .
\end{aligned}
$$

Thus, dividing by $\|v\|_{V}$ and passing to the superior limit, we readily have the bound for $\left\|\partial_{t} \mu(t)\right\|_{V^{*}}$ in terms of $c\left(\left\|\partial_{t} \varphi(t)\right\|+\left\|u_{1}(t)\right\|+1\right)$. Then, by squaring and integrating over $(0, T)$, we deduce (2.20) for $\partial_{t} \mu$. The corresponding property for $\partial_{t} \sigma$ can be obtained in a similar way from (2.10).

ThiRd Estimate: We rewrite (2.18) as

$$
-\Delta \varphi+F_{1, \varepsilon}^{\prime}(\varphi)=\mu+\chi_{\sigma}-\beta \partial_{t} \varphi-F_{2}^{\prime}(\varphi)=: f_{\varphi}
$$

almost everywhere. Due to (2.19) and the Lipschitz continuity of $F_{2}^{\prime}$, we infer that $f_{\varphi}$ is uniformly bounded in $L^{2}(0, T ; H)$. Moreover, let us notice that $F_{1, \varepsilon}^{\prime}: \mathbb{R} \rightarrow \mathbb{R}$ is Lipschitz continuous in $\mathbb{R}$ with $\left(F_{1, \varepsilon}^{\prime}\right)^{\prime}=F_{1, \varepsilon}^{\prime \prime} \in L^{\infty}(\mathbb{R})$. Thus, we may use the known chain rule for generalized derivatives (see, e.g., the comment below the proof of [32], Thm. 7.8) to infer that $\partial_{t}\left(F_{1, \varepsilon}^{\prime}(\varphi)\right)=F_{1, \varepsilon}^{\prime \prime}(\varphi) \partial_{t} \varphi$ a.e. in $Q$, so that it readily follows, along with the above estimates, that $F_{1, \varepsilon}^{\prime}(\varphi) \in L^{2}(0, T ; V)$. Therefore, we can test $(2.21)$ by $F_{1, \varepsilon}^{\prime}(\varphi)$ and integrate by parts in the first term, taking advantage of the homogeneous Neumann boundary condition and obtaining a nonnegative contribution. Thus, by a standard computation it turns out that $F_{1, \varepsilon}^{\prime}(\varphi)$ is bounded in $L^{2}(0, T ; H)$ independently of $\varepsilon$. Then, by comparison in (2.21) and thanks to the elliptic regularity theory, we finally derive that

$$
\left\|F_{1, \varepsilon}^{\prime}\left(\varphi_{\varepsilon}\right)\right\|_{L^{2}(0, T ; H)}+\left\|\varphi_{\varepsilon}\right\|_{L^{2}\left(0, T ; W_{0}\right)} \leq c .
$$

Passage to the Limit: Denote now by $\left(\mu_{\varepsilon}, \varphi_{\varepsilon}, \sigma_{\varepsilon}\right)$ the triplet solving the problem (2.8), (2.18), (2.10), (2.11) with the regularity $(2.5),(2.6)$. Then, in view of the estimates $(2.19),(2.20),(2.22)$, which are independent of $\varepsilon$, by weak and weak-star compactness it turns out that there are $\mu, \varphi, \sigma$ and $\xi$ such that

$$
\begin{aligned}
& \mu_{\varepsilon} \rightarrow \mu \quad \text { weakly star in } H^{1}\left(0, T ; V^{*}\right) \cap L^{\infty}(0, T ; H) \cap L^{2}(0, T ; V), \\
& \varphi_{\varepsilon} \rightarrow \varphi \quad \text { weakly star in } H^{1}(0, T ; H) \cap L^{\infty}(0, T ; V) \cap L^{2}\left(0, T ; W_{0}\right), \\
& \sigma_{\varepsilon} \rightarrow \sigma \quad \text { weakly star in } H^{1}\left(0, T ; V^{*}\right) \cap L^{\infty}(0, T ; H) \cap L^{2}(0, T ; V),
\end{aligned}
$$




$$
F_{1, \varepsilon}^{\prime}\left(\varphi_{\varepsilon}\right) \rightarrow \xi \quad \text { weakly in } L^{2}(0, T ; H)
$$

as $\varepsilon \searrow 0$, possibly along a subsequence. By virtue of (2.23)-(2.25) and the Aubin-Lions lemma (see, e.g., [44], Sect. 8, Cor. 4), we deduce that $\mu_{\varepsilon} \rightarrow \mu, \varphi_{\varepsilon} \rightarrow \varphi, \sigma_{\varepsilon} \rightarrow \sigma$, all strongly in $L^{2}(0, T ; H)$. Then, we can pass to the limit in the variational equalities (2.8), (2.10) and also in (2.18), in order to obtain the equality in (2.9). The nonlinearities $P\left(\varphi_{\varepsilon}\right), \mathbb{h}\left(\varphi_{\varepsilon}\right), F_{2}^{\prime}\left(\varphi_{\varepsilon}\right)$ can be easily taken to the limit, because of the Lipschitz continuity of the involved functions and of the strong convergence of $\varphi_{\varepsilon}$ to $\varphi$ in $L^{2}(0, T ; H)$. In addition, the inclusion in (2.9) results as a consequence of (2.26) and the maximal monotonicity of $\partial F_{1}$, since we can apply, e.g., Lemma 2.3, p. 38 of [1]. Finally, the initial conditions (2.11) can be readily obtained by observing that (2.23)-(2.25) imply weak convergence in $C^{0}([0, T] ; H)$ (actually, even strong for $\varphi_{\varepsilon}$ to $\varphi$ ).

As for uniqueness, it suffices to show that (2.14) is fulfilled for weak solutions. In fact, if we let $\left(\mu_{i}, \varphi_{i}, \xi_{i}, \sigma_{i}\right)$, $i=1,2$, denote two different weak solutions to (1.2)-(1.6) associated with the same initial data $\left(\mu_{0}, \varphi_{0}, \sigma_{0}\right)$ and control variables $\left(u_{1}, u_{2}\right)$, then we derive that (2.14) holds with the right-hand side equal to zero, so that $\varphi_{1}=\varphi_{2}, \mu_{1}=\mu_{2}, \xi_{1}=\xi_{2}, \sigma_{1}=\sigma_{2}$, whence the uniqueness follows.

Continuous dePendence estimate: Now, recalling the notation in the statement of the theorem, we set, for $i=1,2$,

$$
\begin{aligned}
\mu:=\mu_{1}-\mu_{2}, \quad \varphi:=\varphi_{1}-\varphi_{2}, \quad \xi:=\xi_{1}-\xi_{2}, \quad \sigma:=\sigma_{1}-\sigma_{2}, \\
\mu_{0}:=\mu_{0}^{1}-\mu_{0}^{2}, \quad \varphi_{0}:=\varphi_{0}^{1}-\varphi_{0}^{2}, \quad \sigma_{0}:=\sigma_{0}^{1}-\sigma_{0}^{2}, \\
u_{i}:=u_{i}^{1}-u_{i}^{2}, \quad R_{i}:=P\left(\varphi_{i}\right)\left(\sigma_{i}+\chi\left(1-\varphi_{i}\right)-\mu_{i}\right), \quad \mathfrak{h}_{i}:=\mathbb{h}\left(\varphi_{i}\right),
\end{aligned}
$$

and consider the difference of the equations in (2.8)-(2.11) to infer that

$$
\left\langle\partial_{t}(\alpha \mu+\varphi), v\right\rangle+\int_{\Omega} \nabla \mu \cdot \nabla v=\int_{\Omega} \widehat{R} v-\int_{\Omega}\left(\left(\mathbb{h}_{1}-\mathbb{h}_{2}\right) u_{1}^{1}+\mathbb{h}_{2} u_{1}\right) v
$$

for every $v \in V$ and a.e. in $(0, T)$,

$$
\begin{aligned}
& \beta \partial_{t} \varphi-\Delta \varphi+\xi+\left(F_{2}^{\prime}\left(\varphi_{1}\right)-F_{2}^{\prime}\left(\varphi_{2}\right)\right)=\mu+\chi \sigma \text { a.e. in } Q \\
& \left\langle\partial_{t} \sigma, v\right\rangle+\int_{\Omega} \nabla \sigma \cdot \nabla v=\chi \int_{\Omega} \nabla \varphi \cdot \nabla v-\int_{\Omega} \widehat{R} v+\int_{\Omega} u_{2} v
\end{aligned}
$$

for every $v \in V$ and a.e. in $(0, T)$,

$$
\mu(0)=\mu_{0}, \quad \varphi(0)=\varphi_{0}, \quad \sigma(0)=\sigma_{0}, \quad \text { a.e. in } \Omega,
$$

where

$$
\widehat{R}:=R_{1}-R_{2}=\left(P\left(\varphi_{1}\right)-P\left(\varphi_{2}\right)\right)\left(\sigma_{1}+\chi\left(1-\varphi_{1}\right)-\mu_{1}\right)+P\left(\varphi_{2}\right)(\sigma-\chi \varphi-\mu) .
$$

We take $v=\alpha \mu+\varphi$ in (2.27), test (2.28) by $\left(\chi^{2}+\frac{1}{\alpha}\right) \varphi$, and let $v=\sigma$ in (2.29). Then, we add the resulting equalities and integrate over $(0, t)$ and by parts to obtain that

$$
\begin{aligned}
& \frac{1}{2}\|(\alpha \mu+\varphi)(t)\|^{2}+\frac{\beta}{2}\left(\chi^{2}+\frac{1}{\alpha}\right)\|\varphi(t)\|^{2}+\frac{1}{2}\|\sigma(t)\|^{2} \\
& \quad+\alpha \int_{Q_{t}}|\nabla \mu|^{2}+\left(\chi^{2}+\frac{1}{\alpha}\right) \int_{Q_{t}}|\nabla \varphi|^{2}+\left(\chi^{2}+\frac{1}{\alpha}\right) \int_{Q_{t}} \xi \varphi+\int_{Q_{t}}|\nabla \sigma|^{2} \\
& =\frac{1}{2}\left(\left\|\alpha \mu_{0}+\varphi_{0}\right\|^{2}+\beta\left(\chi^{2}+\frac{1}{\alpha}\right)\left\|\varphi_{0}\right\|^{2}+\left\|\sigma_{0}\right\|^{2}\right)-\int_{Q_{t}} \nabla \mu \cdot \nabla \varphi
\end{aligned}
$$




$$
\begin{aligned}
& +\int_{Q_{t}} \widehat{R}(\alpha \mu+\varphi-\sigma)-\int_{Q_{t}}\left(\mathfrak{h}_{1}-\mathbb{h}_{2}\right) u_{1}^{1}(\alpha \mu+\varphi)-\int_{Q_{t}} \mathbb{h}_{2} u_{1}(\alpha \mu+\varphi) \\
& -\left(\chi^{2}+\frac{1}{\alpha}\right) \int_{Q_{t}}\left(F_{2}^{\prime}\left(\varphi_{1}\right)-F_{2}^{\prime}\left(\varphi_{2}\right)\right) \varphi+\left(\chi^{2}+\frac{1}{\alpha}\right) \int_{Q_{t}} \frac{1}{\alpha}(\alpha \mu+\varphi) \varphi \\
& -\frac{1}{\alpha}\left(\chi^{2}+\frac{1}{\alpha}\right) \int_{Q_{t}}|\varphi|^{2}+\left(\chi^{2}+\frac{1}{\alpha}\right) \chi \int_{Q_{t}} \sigma \varphi+\chi \int_{Q_{t}} \nabla \varphi \cdot \nabla \sigma+\int_{Q_{t}} u_{2} \sigma
\end{aligned}
$$

for a.e. $t \in(0, T)$, where we also used that $\mu=\frac{1}{\alpha}(\alpha \mu+\varphi)-\frac{1}{\alpha} \varphi$. Observe that all of the terms on the lefthand side are nonnegative; in particular, the sixth is nonnegative thanks to the monotonicity of $\partial F_{1}$. Next, we denote by $I_{1}, \ldots, I_{11}$ the eleven terms on the right-hand side of $(2.31)$, in the above order, and estimate them individually. Using the Young inequality, we infer that

$$
I_{2}+I_{10} \leq \frac{1}{2}\left(\chi^{2}+\frac{1}{\alpha}\right) \int_{Q_{t}}|\nabla \varphi|^{2}+\frac{\alpha}{2} \int_{Q_{t}}|\nabla \mu|^{2}+\frac{1}{2} \int_{Q_{t}}|\nabla \sigma|^{2},
$$

and here the last three contributions on the right-hand side can be absorbed on the left-hand side of (2.31). We also immediately observe that $I_{8} \leq 0$. Moreover, with the help of (W2), (W4), and recalling (2.4), we deduce from Young's inequality that

$$
\begin{aligned}
I_{4}+I_{5} & \leq L\left\|u_{1}^{1}\right\|_{L^{\infty}(Q)} \int_{Q_{t}}|\varphi||\alpha \mu+\varphi|+\left\|\mathrm{h}_{2}\right\|_{\infty} \int_{Q_{t}}\left|u_{1}\right||\alpha \mu+\varphi| \\
& \leq c \int_{Q_{t}}\left(|\alpha \mu+\varphi|^{2}+|\varphi|^{2}+\left|u_{1}\right|^{2}\right)
\end{aligned}
$$

as well as

$$
\begin{aligned}
I_{6}+ & I_{7}+I_{9}+I_{11} \\
\leq & L\left(\chi^{2}+\frac{1}{\alpha}\right) \int_{Q_{t}}|\varphi|^{2}+\frac{1}{2 \alpha}\left(\chi^{2}+\frac{1}{\alpha}\right) \int_{Q_{t}}\left(|\alpha \mu+\varphi|^{2}+|\varphi|^{2}\right) \\
& +\frac{\chi}{2}\left(\chi^{2}+\frac{1}{\alpha}\right) \int_{Q_{t}}\left(|\sigma|^{2}+|\varphi|^{2}\right)+\frac{1}{2} \int_{Q_{t}}\left(\left|u_{2}\right|^{2}+|\sigma|^{2}\right) \\
\leq & c \int_{Q_{t}}\left(|\varphi|^{2}+|\alpha \mu+\varphi|^{2}+|\sigma|^{2}+\left|u_{2}\right|^{2}\right) .
\end{aligned}
$$

It remains to estimate $I_{3}$. Using the boundedness and Lipschitz continuity of $P$, the Hölder and Young inequalities, and the continuous embedding $V \subset L^{4}(\Omega)$, we find that

$$
\begin{aligned}
I_{3} \leq & L \int_{Q_{t}}|\varphi|\left(\left|\sigma_{1}\right|+\chi+\chi\left|\varphi_{1}\right|+\left|\mu_{1}\right|\right)(|\alpha \mu+\varphi|+|\sigma|) \\
& +\|P\|_{\infty} \int_{Q_{t}}\left(|\sigma|+\frac{1}{\alpha}|\alpha \mu+\varphi|+\left(\chi+\frac{1}{\alpha}\right)|\varphi|\right)(|\alpha \mu+\varphi|+|\sigma|) \\
\leq & c \int_{0}^{t}\|\varphi(s)\|_{4}\left(\left\|\sigma_{1}\right\|_{4}+\left\|\varphi_{1}\right\|_{4}+\left\|\mu_{1}\right\|_{4}\right)(s)(\|\alpha \mu+\varphi\|+\|\sigma\|)(s) \mathrm{d} s \\
& +c \int_{0}^{t}\|\varphi(s)\|(\|\alpha \mu+\varphi\|+\|\sigma\|)(s) \mathrm{d} s+c \int_{Q_{t}}\left(|\sigma|^{2}+|\alpha \mu+\varphi|^{2}+|\varphi|^{2}\right)
\end{aligned}
$$




$$
\begin{aligned}
\leq & \delta \int_{0}^{t}\|\varphi(s)\|_{V}^{2} \mathrm{~d} s+c_{\delta} \int_{0}^{t}\left(\left(\left\|\sigma_{1}\right\|_{V}^{2}+\left\|\varphi_{1}\right\|_{V}^{2}+\left\|\mu_{1}\right\|_{V}^{2}\right)\left(\|\alpha \mu+\varphi\|^{2}+\|\sigma\|^{2}\right)\right)(s) \mathrm{d} s \\
& +c \int_{Q_{t}}\left(|\sigma|^{2}+|\alpha \mu+\varphi|^{2}+|\varphi|^{2}\right),
\end{aligned}
$$

for a positive $\delta$ to be chosen, for instance, less than or equal to $\frac{1}{4}\left(\chi^{2}+\frac{1}{\alpha}\right)$. Since $\left(\mu_{1}, \varphi_{1}, \xi_{1}, \sigma_{1}\right)$ is a weak solution to (1.2)-(1.6) in the sense of Definition 2.1, it follows that the function

$$
t \mapsto\left(\left\|\sigma_{1}(t)\right\|_{V}^{2}+\left\|\varphi_{1}(t)\right\|_{V}^{2}+\left\|\mu_{1}(t)\right\|_{V}^{2}\right)
$$

belongs to $L^{1}(0, T)$. Hence, we can collect all the above inequalities and apply the Gronwall lemma to finally derive the estimate $(2.14)$.

Since the control problem introduced above will demand strong regularities, we also prove the existence of strong solutions (i.e., regularity results for our weak solutions) to the system (1.2)-(1.6) under further assumptions. In this direction, in addition to (W1)-(W4), we postulate that:

(S1) $F=F_{1}+F_{2} ; F_{1}: \mathbb{R} \rightarrow[0,+\infty]$ is convex and lower semicontinuous with $F_{1}(0)=0 ; F_{2} \in C^{5}(\mathbb{R})$, and $F_{2}^{\prime}$ is Lipschitz continuous on $\mathbb{R}$.

(S2) There exists an interval $\left(r_{-}, r_{+}\right)$with $-\infty \leq r_{-}<0<r_{+} \leq+\infty$ such that the restriction of $F_{1}$ to $\left(r_{-}, r_{+}\right)$belongs to $C^{5}\left(r_{-}, r_{+}\right)$.

(S3) It holds $\lim _{r \searrow r_{-}} F^{\prime}(r)=-\infty$ and $\lim _{r} \nearrow_{r_{+}} F^{\prime}(r)=+\infty$.

(S4) $P, \boldsymbol{h} \in C^{3}(\mathbb{R}) \cap W^{3, \infty}(\mathbb{R})$, and $\mathbb{h}$ is positive on $\left(r_{-}, r_{+}\right)$.

Observe that (S4) entails that $P, P^{\prime}, P^{\prime \prime}, \mathfrak{h}, \mathbb{h}^{\prime}, \mathbb{h}^{\prime \prime}$ are Lipschitz continuous on $\mathbb{R}$. Moreover, let us remark that the above setting allows us to include the singular logarithmic potential (1.10) and the associated quartic approximation (1.9), but it excludes the double obstacle potential (1.11), which cannot be considered in the framework of (S2)-(S3). Furthermore, the prescribed regularity for the potential $F$ entails that its derivative can be defined in the classical manner so that we no longer need considering a selection $\xi$ in the notion of strong solution below. Moreover, it will be useful to set, for a fixed $R>0$,

$$
\mathcal{U}_{R}:=\left\{\mathbf{u}=\left(u_{1}, u_{2}\right) \in L^{\infty}(Q)^{2}:\|\mathbf{u}\|_{\infty}<R\right\}
$$

Under these conditions, we have the following result concerning the well-posedness of the state system (1.2)-(1.6), where the equations and conditions have to be fulfilled almost everywhere in $Q$.

Theorem 2.3 (Strong well-posedness). Suppose that the conditions (W1), (S1)-(S4), and (2.32) are fulfilled. Moreover, let the initial data fulfill

$$
\mu_{0}, \sigma_{0} \in H^{1}(\Omega) \cap L^{\infty}(\Omega), \quad \varphi_{0} \in W_{0},
$$

as well as

$$
r_{-}<\min _{x \in \bar{\Omega}} \varphi_{0}(x) \leq \max _{x \in \bar{\Omega}} \varphi_{0}(x)<r_{+}
$$

Then the state system (1.2)-(1.6) has for every $\mathbf{u}=\left(u_{1}, u_{2}\right) \in \mathcal{U}_{R}$ a unique solution $(\mu, \varphi, \sigma)$ with the regularity

$$
\begin{aligned}
& \mu \in H^{1}(0, T ; H) \cap C^{0}([0, T] ; V) \cap L^{2}\left(0, T ; W_{0}\right) \cap L^{\infty}(Q), \\
& \varphi \in W^{1, \infty}(0, T ; H) \cap H^{1}(0, T ; V) \cap L^{\infty}\left(0, T ; W_{0}\right) \cap C^{0}(\bar{Q}),
\end{aligned}
$$




$$
\sigma \in H^{1}(0, T ; H) \cap C^{0}([0, T] ; V) \cap L^{2}\left(0, T ; W_{0}\right) \cap L^{\infty}(Q) .
$$

Moreover, there is a constant $K_{1}>0$, which depends on $\Omega, T, R, \alpha, \beta$ and the data of the system, but not on the choice of $\mathbf{u} \in \mathcal{U}_{R}$, such that

$$
\begin{aligned}
& \|\mu\|_{H^{1}(0, T ; H) \cap C^{0}([0, T] ; V) \cap L^{2}\left(0, T ; W_{0}\right) \cap L^{\infty}(Q)} \\
& +\|\varphi\|_{W^{1, \infty}(0, T ; H) \cap H^{1}(0, T ; V) \cap L^{\infty}\left(0, T ; W_{0}\right) \cap C^{0}(\bar{Q})} \\
& +\|\sigma\|_{H^{1}(0, T ; H) \cap C^{0}([0, T] ; V) \cap L^{2}\left(0, T ; W_{0}\right) \cap L^{\infty}(Q)} \leq K_{1} .
\end{aligned}
$$

Furthermore, there exist two values $r_{*}, r^{*}$, depending on $\Omega, T, R, \alpha, \beta$ and the data of the system, but not on the choice of $\mathbf{u} \in \mathfrak{U}_{R}$, such that

$$
r_{-}<r_{*} \leq \varphi(x, t) \leq r^{*}<r_{+} \quad \text { for all }(x, t) \in \bar{Q} .
$$

Also, there is some constant $K_{2}>0$, with the same dependencies as $K_{1}$, such that

$$
\begin{aligned}
& \max _{i=0,1,2,3}\left\|P^{(i)}(\varphi)\right\|_{L^{\infty}(Q)}+\max _{i=0,1,2,3}\left\|\mathfrak{h}^{(i)}(\varphi)\right\|_{L^{\infty}(Q)} \\
& +\max _{i=0,1,2,3,4,5}\left\|F^{(i)}(\varphi)\right\|_{L^{\infty}(Q)} \leq K_{2} .
\end{aligned}
$$

Finally, for $i=1,2$, let $\left(\mu_{i}, \varphi_{i}, \sigma_{i}\right)$ be a strong solution to (1.2)-(1.6) associated with the initial data $\left(\mu_{0}^{i}, \varphi_{0}^{i}, \sigma_{0}^{i}\right)$ satisfying (2.33)-(2.34) and the controls $\mathbf{u}^{i}=\left(u_{1}^{i}, u_{2}^{i}\right) \in \mathfrak{U}_{R}$. Then, there is a positive constant $C_{D}$, depending only on data, such that

$$
\begin{aligned}
& \left\|\mu_{1}-\mu_{2}\right\|_{H^{1}(0, T ; H) \cap L^{\infty}(0, T ; V) \cap L^{2}\left(0, T ; W_{0}\right)}+\left\|\varphi_{1}-\varphi_{2}\right\|_{H^{1}(0, T ; H) \cap L^{\infty}(0, T ; V) \cap L^{2}\left(0, T ; W_{0}\right)} \\
& \quad+\left\|\sigma_{1}-\sigma_{2}\right\|_{H^{1}(0, T ; H) \cap L^{\infty}(0, T ; V) \cap L^{2}\left(0, T ; W_{0}\right)} \\
& \leq C_{D}\left(\left\|\mu_{0}^{1}-\mu_{0}^{2}\right\|_{V}+\left\|\varphi_{0}^{1}-\varphi_{0}^{2}\right\|_{V}+\left\|\sigma_{0}^{1}-\sigma_{0}^{2}\right\|_{V}+\left\|\mathbf{u}^{1}-\mathbf{u}^{2}\right\|_{L^{2}(0, T ; H)^{2}}\right) .
\end{aligned}
$$

Remark 2.4. (i) The separation property (2.39) is particularly important for the case of singular potentials such as $F_{\log }$. Indeed, it guarantees that the phase variable always stays away from the critical values $r_{-}, r_{+}$ that may correspond to the pure phases. In this way, the singularity is no longer an obstacle for the analysis; indeed, the values of $\varphi$ range in some interval in which $F_{1}$ is smooth.

(ii) Notice that (2.34) entails that $F^{(i)}\left(\varphi_{0}\right) \in C^{0}(\bar{\Omega})$ for $i=0,1, \ldots, 5$. This condition can be restrictive for singular potentials; for instance, in the case of $F_{\log }$ we have $r_{ \pm}= \pm 1$, so that (2.34) excludes the pure phases (tumor and healthy tissue) as initial data.

Notice also that, owing to Definition 2.1, the control-to-state operator

$$
\mathcal{S}: \mathbf{u}=\left(u_{1}, u_{2}\right) \mapsto(\mu, \varphi, \sigma)
$$

is well defined as a mapping between $\mathcal{U}=L^{\infty}(Q)^{2}$ and the Banach space specified by the regularity results (2.35)-(2.37). Actually, the control-to-state operator $\mathcal{S}$ may be well defined just after Theorem 2.2, but the notion of weak solutions proposed there ( $c f$. Def. 2.1) would not suffice for the investigation of the optimal control problem (eP).

Proof. Again, we proceed formally, but still using the Yosida approximation $F_{1, \varepsilon}^{\prime}$, at least in the first part of the proof. Of course, we take for granted all the estimates already done in the existence proof for Theorem 2.2, and start now with additional estimates independent of $\varepsilon$. To avoid a heavy notation, we proceed as in Theorem 2.2 
and use the simpler notation $(\mu, \varphi, \sigma)$ for the variables of the approximated system instead of $\left(\mu_{\varepsilon}, \varphi_{\varepsilon}, \sigma_{\varepsilon}\right)$, while we will reintroduce the correct notation exhibiting the dependence of $\varepsilon$ at the end of each estimate.

FIRST ESTIMATE: We rewrite the variational equality (2.8) as

$$
\alpha\left\langle\partial_{t} \mu, v\right\rangle+\int_{\Omega} \nabla \mu \cdot \nabla v=\int_{\Omega} f_{\mu} v \quad \text { for every } v \in V \text { and a.e. in }(0, T),
$$

where $f_{\mu}:=-\partial_{t} \varphi+P(\varphi)(\sigma+\chi(1-\varphi)-\mu)-\mathbb{h}(\varphi) u_{1}$ is already known to be uniformly bounded in $L^{2}(0, T ; H)$ by (2.19). As $\mu(0)=\mu_{0}$ is now in $H^{1}(\Omega)$, it follows from the regularity theory for parabolic problems (see, e.g., [37]) that

$$
\left\|\mu_{\varepsilon}\right\|_{H^{1}(0, T ; H) \cap L^{\infty}(0, T ; V) \cap L^{2}\left(0, T ; W_{0}\right)} \leq c,
$$

and (2.42) can be equivalently rewritten as the equation (1.2) along with the Neumann boundary condition $\partial_{\mathbf{n}} \mu=0$ a.e. on $\Sigma$. Next, recalling also (2.22) and arguing similarly for the variational equality (2.10), rewritten as

$$
\begin{aligned}
& \left\langle\partial_{t} \sigma, v\right\rangle+\int_{\Omega} \nabla \sigma \cdot \nabla v=-\int_{\Omega}\left(\chi \Delta \varphi+P(\varphi)(\sigma+\chi(1-\varphi)-\mu)-u_{2}\right) v \\
& \quad \text { for every } v \in V \text { and a.e. in }(0, T),
\end{aligned}
$$

we also deduce that

$$
\left\|\sigma_{\varepsilon}\right\|_{H^{1}(0, T ; H) \cap L^{\infty}(0, T ; V) \cap L^{2}\left(0, T ; W_{0}\right)} \leq c .
$$

Hence, (1.4) holds a.e. in $Q$, and all of the boundary conditions in (1.5) hold a.e. on $\Sigma$.

SECOnd estimate: We formally differentiate (2.18) with respect to time, obtaining

$$
\beta \partial_{t}\left(\partial_{t} \varphi\right)-\Delta\left(\partial_{t} \varphi\right)+F_{1, \varepsilon}^{\prime \prime}(\varphi) \partial_{t} \varphi=\partial_{t} \mu+\chi \partial_{t} \sigma-F_{2}^{\prime \prime}(\varphi) \partial_{t} \varphi=: g_{\varphi}
$$

where $g_{\varphi}$ is bounded in $L^{2}(0, T ; H)$ independently of $\varepsilon$, on account of (2.43), (2.44), (S1), and (2.19) (indeed, $F_{2}^{\prime \prime}$ is globally bounded on $\mathbb{R}$ ). Then, multiplying (2.45) by $\partial_{t} \varphi$ and integrating over $\Omega$ and by parts, we find that

$$
\frac{\beta}{2} \frac{\mathrm{d}}{\mathrm{d} t}\left\|\partial_{t} \varphi\right\|^{2}+\left\|\nabla \partial_{t} \varphi\right\|^{2}+\int_{\Omega} F_{1, \varepsilon}^{\prime \prime}(\varphi)\left|\partial_{t} \varphi\right|^{2} \leq\left\|g_{\varphi}\right\|\left\|\partial_{t} \varphi\right\|, \quad \text { a.e. in }(0, T)
$$

where the third term on the left-hand side is nonnegative owing to the monotonicity of $F_{1, \varepsilon}^{\prime}$. Now, we aim to integrate (2.46) with respect to time. Note that taking $t=0$ in (2.18) produces

$$
\partial_{t} \varphi(0)=\frac{1}{\beta}\left(\Delta \varphi_{0}-F_{1, \varepsilon}^{\prime}\left(\varphi_{0}\right)+\mu_{0}+\chi \sigma_{0}-F_{2}^{\prime}\left(\varphi_{0}\right)\right)
$$

where the right-hand side is bounded in $H$ by virtue of (2.17), (2.33), (2.34), and (S1). Hence, we can integrate $(2.46)$ over $[0, t]$, with $t \in(0, T]$, to conclude that

$$
\left\|\varphi_{\varepsilon}\right\|_{W^{1, \infty}(0, T ; H) \cap H^{1}(0, T ; V)} \leq c .
$$

THIRD ESTimate: We come back to the elliptic equation (2.21) and observe that now we have at hand that $f_{\varphi}$ is bounded in $L^{\infty}(0, T ; H)$. Then, arguing similarly as in the proof of $(2.22)$, using monotonicity and elliptic 
regularity theory, we easily infer that

$$
\left\|F_{1, \varepsilon}^{\prime}\left(\varphi_{\varepsilon}\right)\right\|_{L^{\infty}(0, T ; H)}+\left\|\varphi_{\varepsilon}\right\|_{L^{\infty}\left(0, T ; W_{0}\right)} \leq c,
$$

so that the continuity of the embedding $W_{0} \subset C^{0}(\bar{\Omega})$ entails that

$$
\left\|\varphi_{\varepsilon}\right\|_{L^{\infty}(Q)} \leq c
$$

Fourth estimate: Next, we consider the parabolic equation (1.2), written as

$$
\alpha \partial_{t} \mu-\Delta \mu=-\partial_{t} \varphi+P(\varphi)(\sigma+\chi(1-\varphi)-\mu)-\mathfrak{h}(\varphi) u_{1}=: f_{\mu}
$$

and observe that now, thanks to (2.47), we have that $\partial_{t} \varphi$, and consequently $f_{\mu}$, are bounded in $L^{\infty}(0, T ; H)$. Moreover, we recall (2.33) and note that $\mu_{0} \in L^{\infty}(\Omega)$, in particular. Thus, we can apply the regularity result ([36], Thm. 7.1, p. 181) to show that

$$
\left\|\mu_{\varepsilon}\right\|_{L^{\infty}(Q)} \leq c
$$

With similar arguments we can easily obtain the same property for the nutrient variable. In fact, it suffices to rewrite (1.4) as a parabolic equation with forcing term

$$
f_{\sigma}=-\chi \Delta \varphi+P(\varphi)(\sigma+\chi(1-\varphi)-\mu)+u_{2},
$$

and notice that (2.48) allows us to infer that $\Delta \varphi$, and thus $f_{\sigma}$, are bounded in $L^{\infty}(0, T ; H)$. Hence, we can apply the same argument to conclude that

$$
\left\|\sigma_{\varepsilon}\right\|_{L^{\infty}(Q)} \leq c
$$

Now, we collect the estimates (2.43)-(2.44), (2.47)-(2.51) and point out that they still hold for the real solution $(\mu, \varphi, \sigma)$ when passing to the limit as $\varepsilon \searrow 0$, because of the weak or weak star lower semicontinuity of norms. Then, we realize that indeed the global estimate (2.38) in the statement has been proved, with the observation that $L^{\infty}(Q)$ for $\varphi$ is replaced by $C^{0}(\bar{Q})$, since this continuity property is actually ensured by

$$
\varphi \in W^{1, \infty}(0, T ; H) \cap L^{\infty}\left(0, T ; W_{0}\right)
$$

and the compact embedding $W_{0} \subset C^{0}(\bar{\Omega})$ (see, e.g., [44], Sect. 8, Cor. 4).

SEPARATion PRoperty: At this point, the equation (1.3) holds for the limit functions, with the datum $F^{\prime}=$ $F_{1}^{\prime}+F_{2}^{\prime}$ as in (S1)-(S3) and with the right-hand side bounded in $L^{\infty}(Q)$. Thus, there exists a positive constant $C_{*}$ for which

$$
\|\mu+\chi \sigma\|_{L^{\infty}(Q)} \leq C_{*} .
$$

Moreover, the condition (2.34) for the initial $\varphi_{0}$ and the growth assumption (S3) imply the existence of some constants $r_{*}$ and $r^{*}$ such that $r_{-}<r_{*} \leq r^{*}<r_{+}$and

$$
\begin{gathered}
r_{*} \leq \inf _{x \in \Omega} \operatorname{ess} \varphi_{0}(x), \quad r^{*} \geq \sup _{x \in \Omega} \operatorname{ess} \varphi_{0}(x), \\
F^{\prime}(r)+C_{*} \leq 0 \quad \forall r \in\left(r_{-}, r_{*}\right), \quad F^{\prime}(r)-C_{*} \geq 0 \quad \forall r \in\left(r^{*}, r_{+}\right) .
\end{gathered}
$$


Then, let us multiply (1.3) by $v=\left(\varphi-r^{*}\right)^{+}-\left(\varphi-r_{*}\right)^{-}$, where the standard positive $(\cdot)^{+}$and negative $(\cdot)^{-}$ parts are used here. Then, we integrate over $Q_{t}=\Omega \times(0, t)$, for $t \in(0, T]$, and with the help of (2.52) deduce that

$$
\begin{aligned}
& \frac{\beta}{2}\|v(t)\|^{2}+\int_{Q_{t}}|\nabla v|^{2} \\
& \quad=\int_{Q_{t} \cap\left\{\varphi<r_{*}\right\}}\left(F^{\prime}(\varphi)-\mu-\chi \sigma\right)\left(r_{*}-\varphi\right)+\int_{Q_{t} \cap\left\{\varphi>r^{*}\right\}}\left(\mu+\chi \sigma-F^{\prime}(\varphi)\right)\left(\varphi-r^{*}\right) \\
& \quad \leq \int_{Q_{t} \cap\left\{\varphi<r_{*}\right\}}\left(F^{\prime}(\varphi)+C_{*}\right)\left(r_{*}-\varphi\right)+\int_{Q_{t} \cap\left\{\varphi>r^{*}\right\}}\left(C_{*}-F^{\prime}(\varphi)\right)\left(\varphi-r^{*}\right),
\end{aligned}
$$

where we also applied (2.53) to have that $v(0)=0$. Note that the right-hand side above is nonpositive due to (2.54), so that $v=0$ almost everywhere, which in turn implies that

$$
r_{*} \leq \varphi \leq r^{*} \text { a.e. in } Q \text {. }
$$

Then, (2.39) is proven, and, at this point, the assumptions (S1)-(S4) enable us to directly deduce (2.40).

On account of the above regularity, the separation property, and the assumptions (S1)-(S4), we are now in a position to show the refined continuous dependence estimate given by (2.41). In this direction, we employ the notation introduced in the proof of Theorem 2.2 and consider the system of the differences (2.27)-(2.30). Notice that we now have that $F$ is differentiable, so that $\xi+\left(F_{2}^{\prime}\left(\varphi_{1}\right)-F_{2}^{\prime}\left(\varphi_{2}\right)\right)=F^{\prime}\left(\varphi_{1}\right)-F^{\prime}\left(\varphi_{2}\right)$. Moreover, let us remark that due to the separation property (2.39) and to the reinforced assumptions (S1)-(S3), it follows that $F^{\prime}$ is Lipschitz continuous in the range of the occurring arguments.

First estimate: We test (2.27) by $\mu,(2.28)$ - to which we add the term $\varphi$ on both sides - by $\partial_{t} \varphi$, as well as (2.29) by $\sigma$. Then, we sum up and integrate over $Q_{t}$ and by parts. With the help of the cancellation of two terms we deduce that

$$
\begin{aligned}
\frac{\alpha}{2}\|\mu(t)\|^{2}+\int_{Q_{t}}|\nabla \mu|^{2}+\beta \int_{Q_{t}}\left|\partial_{t} \varphi\right|^{2}+\frac{1}{2}\|\varphi(t)\|_{V}^{2}+\frac{1}{2}\|\sigma(t)\|^{2}+\int_{Q_{t}}|\nabla \sigma|^{2} \\
=\frac{1}{2}\left(\alpha\left\|\mu_{0}\right\|^{2}+\left\|\varphi_{0}\right\|_{V}^{2}+\left\|\sigma_{0}\right\|^{2}\right)+\int_{Q_{t}} \widehat{R}(\mu-\sigma)-\int_{Q_{t}}\left(\mathbb{h}_{1}-\mathbb{h}_{2}\right) u_{1}^{1} \mu \\
\quad-\int_{Q_{t}} \mathbb{h}_{2} u_{1} \mu-\int_{Q_{t}}\left(F^{\prime}\left(\varphi_{1}\right)-F^{\prime}\left(\varphi_{2}\right)-\varphi\right) \partial_{t} \varphi+\int_{Q_{t}} \mu \partial_{t} \varphi \\
+\chi \int_{Q_{t}} \sigma \partial_{t} \varphi+\chi \int_{Q_{t}} \nabla \varphi \cdot \nabla \sigma+\int_{Q_{t}} u_{2} \sigma=: I_{1}+\ldots+I_{9} .
\end{aligned}
$$

Using the Young and Hölder inequalities, the Lipschitz continuity and boundedness of $P$ along with the strong regularity (2.38) of the solutions, we infer that

$$
\begin{aligned}
I_{2} \leq & L \int_{Q_{t}}|\varphi|\left(\left|\sigma_{1}\right|+\chi+\chi\left|\varphi_{1}\right|+\left|\mu_{1}\right|\right)(|\mu|+|\sigma|) \\
& +\|P\|_{\infty} \int_{Q_{t}}(|\sigma|+\chi|\varphi|+|\mu|)(|\mu|+|\sigma|) \\
\leq & c\left(\left\|\sigma_{1}\right\|_{L^{\infty}(Q)}^{2}+1+\left\|\varphi_{1}\right\|_{L^{\infty}(Q)}^{2}+\left\|\mu_{1}\right\|_{L^{\infty}(Q)}^{2}\right) \int_{0}^{t}\|\varphi(s)\|^{2} \mathrm{~d} s \\
& +c \int_{Q_{t}}\left(|\mu|^{2}+|\varphi|^{2}+|\sigma|^{2}\right) .
\end{aligned}
$$


Recalling (2.32), we have that

$$
\begin{aligned}
& I_{3} \leq \int_{Q_{t}}\left|\mathbb{h}\left(\varphi_{1}\right)-\mathbb{h}\left(\varphi_{2}\right)\right|\left|u_{1}^{1}\right||\mu| \\
& \leq L \int_{0}^{t}\|\varphi(s)\|\left\|u_{1}^{1}(s)\right\|_{\infty}\|\mu(s)\| \mathrm{d} s \leq \frac{L R}{2} \int_{Q_{t}}\left(|\varphi|^{2}+|\mu|^{2}\right) .
\end{aligned}
$$

Moreover, it is easy to see that

$$
\begin{aligned}
& I_{4} \leq c \int_{Q_{t}}\left(|\mu|^{2}+\left|u_{1}\right|^{2}\right), \quad I_{5} \leq \frac{\beta}{4} \int_{Q_{t}}\left|\partial_{t} \varphi\right|^{2}+c \int_{Q_{t}}|\varphi|^{2}, \\
& I_{6}+I_{7} \leq \frac{\beta}{4} \int_{Q_{t}}\left|\partial_{t} \varphi\right|^{2}+c \int_{Q_{t}}\left(|\mu|^{2}+|\sigma|^{2}\right), \\
& I_{8} \leq \frac{1}{2} \int_{Q_{t}}|\nabla \sigma|^{2}+c \int_{Q_{t}}|\nabla \varphi|^{2}, \quad I_{9} \leq c \int_{Q_{t}}\left(|\sigma|^{2}+\left|u_{2}\right|^{2}\right) .
\end{aligned}
$$

Hence, we can collect the inequalities and apply the Gronwall lemma to infer that the differences satisfy

$$
\begin{aligned}
& \|\mu\|_{L^{\infty}(0, T ; H) \cap L^{2}(0, T ; V)}+\|\varphi\|_{H^{1}(0, T ; H) \cap L^{\infty}(0, T ; V)}+\|\sigma\|_{L^{\infty}(0, T ; H) \cap L^{2}(0, T ; V)} \\
& \quad \leq c\left(\left\|\mu_{0}\right\|+\left\|\varphi_{0}\right\|_{V}+\left\|\sigma_{0}\right\|+\left\|u_{1}\right\|_{L^{2}(0, T ; H)}+\left\|u_{2}\right\|_{L^{2}(0, T ; H)}\right) .
\end{aligned}
$$

SECOND ESTimate: By exploiting the ellipticity of equation (2.28), the Lipschitz continuity of $F^{\prime}$, along with the above estimates, it is straightforward to derive that

$$
\|\varphi\|_{L^{2}\left(0, T ; W_{0}\right)} \leq c\left(\left\|\mu_{0}\right\|+\left\|\varphi_{0}\right\|_{V}+\left\|\sigma_{0}\right\|+\left\|u_{1}\right\|_{L^{2}(0, T ; H)}+\left\|u_{2}\right\|_{L^{2}(0, T ; H)}\right)
$$

ThiRd estimate: We argue in a similar way as in (2.42) and rewrite (2.27) as a parabolic variational equality for $\mu=\mu_{1}-\mu_{2}$ with source term given by

$$
\begin{aligned}
f_{\mu}:= & -\partial_{t} \varphi+\left(P\left(\varphi_{1}\right)-P\left(\varphi_{2}\right)\right)\left(\sigma_{1}+\chi\left(1-\varphi_{1}\right)-\mu_{1}\right)+P\left(\varphi_{2}\right)(\sigma-\chi \varphi-\mu) \\
& -\left(\mathbb{h}_{1}-\mathbb{h}_{2}\right) u_{1}^{1}+\mathbb{h}_{2} u_{1} .
\end{aligned}
$$

On account of (2.38) and the above estimates, we easily deduce that

$$
\left\|f_{\mu}\right\|_{L^{2}(0, T ; H)} \leq c\left(\left\|\mu_{0}\right\|+\left\|\varphi_{0}\right\|_{V}+\left\|\sigma_{0}\right\|+\left\|u_{1}\right\|_{L^{2}(0, T ; H)}+\left\|u_{2}\right\|_{L^{2}(0, T ; H)}\right) .
$$

Hence, observing that $\mu(0)=\mu_{0}=\mu_{0}^{1}-\mu_{0}^{2}$ is in $H^{1}(\Omega)$, we can readily infer from the parabolic regularity theory (see, e.g., [37]) that

$$
\begin{aligned}
& \|\mu\|_{H^{1}(0, T ; H) \cap L^{\infty}(0, T ; V) \cap L^{2}\left(0, T ; W_{0}\right)} \\
& \quad \leq c\left(\left\|\mu_{0}\right\|_{V}+\left\|\varphi_{0}\right\|_{V}+\left\|\sigma_{0}\right\|+\left\|u_{1}\right\|_{L^{2}(0, T ; H)}+\left\|u_{2}\right\|_{L^{2}(0, T ; H)}\right) .
\end{aligned}
$$

FourTh ESTimate: Arguing in a similar manner for the equality (2.29), we infer that the right-hand side can be rewritten as $\int_{\Omega} f_{\sigma} v$, with

$$
f_{\sigma}:=-\chi \Delta \varphi-\left(P\left(\varphi_{1}\right)-P\left(\varphi_{2}\right)\right)\left(\sigma_{1}+\chi\left(1-\varphi_{1}\right)-\mu_{1}\right)-P\left(\varphi_{2}\right)(\sigma-\chi \varphi-\mu)+u_{2} .
$$


Note that $\left\|f_{\sigma}\right\|_{L^{2}(0, T ; H)}$ is again already bounded as above and $\sigma(0)=\sigma_{0}=\sigma_{0}^{1}-\sigma_{0}^{2}$ is in $H^{1}(\Omega)$, so that

$$
\begin{aligned}
& \|\sigma\|_{H^{1}(0, T ; H) \cap L^{\infty}(0, T ; V) \cap L^{2}\left(0, T ; W_{0}\right)} \\
& \quad \leq c\left(\left\|\mu_{0}\right\|+\left\|\varphi_{0}\right\|_{V}+\left\|\sigma_{0}\right\|_{V}+\left\|u_{1}\right\|_{L^{2}(0, T ; H)}+\left\|u_{2}\right\|_{L^{2}(0, T ; H)}\right) .
\end{aligned}
$$

By collecting (2.56)-(2.59), we finally conclude the proof of the assertion.

\section{EXISTENCE OF A MinimizeR}

Now that the well-posedness results for system (1.2)-(1.6) have been addressed, we can deal with a corresponding optimal control problem, where the source terms $u_{1}$ and $u_{2}$ act as controls. In this direction, we require that the cost functional $\mathcal{J}$ is defined by (1.1) and that the following assumptions are fulfilled:

(C1) $b_{1}, b_{2}$ are nonnegative constants, and $b_{0}$ is positive.

(C2) $\widehat{\varphi}_{Q} \in L^{2}(Q)$ and $\widehat{\varphi}_{\Omega} \in L^{2}(\Omega)$.

(C3) $\mathcal{U}_{\mathrm{ad}}=\left\{\mathbf{u}=\left(u_{1}, u_{2}\right) \in \mathcal{U}: \underline{u}_{i} \leq u_{i} \leq \widehat{u}_{i}\right.$ a.e. in $\left.Q, i=1,2\right\}$, where $\underline{u}_{i}, \widehat{u}_{i} \in L^{\infty}(Q)$ satisfy $\underline{u}_{i} \leq \widehat{u}_{i}$ a.e. in $Q, i=1,2$.

Notice that $\mathcal{U}_{\mathrm{ad}}$ is a nonempty, closed and convex subset of $\mathcal{U}=L^{\infty}(Q)^{2}$. In the following, it will sometimes be necessary to work with a bounded open superset of $\mathcal{U}_{\text {ad }}$. We therefore once and for all fix some $R>0$ such that $\mathcal{U}_{R} \supset \mathcal{U}_{\mathrm{ad}}$, where $\mathcal{U}_{R}$ is defined by (2.32). The first result for ( $\left.\mathcal{C P}\right)$ concerns the existence of a minimizer, where the proof readily follows from the direct method of calculus of variations, along with weak and weak star compactness arguments.

Theorem 3.1 (Existence of minimizers). Assume that (W1), (S1)-(S4), (2.33), (2.34), and (C1)-(C3) hold true. Then the minimization problem (eP) admits a minimizer.

Proof. At first, let us notice that $\mathcal{J}$ is nonnegative, so that we can pick a minimizing sequence $\left\{\mathbf{u}_{n}\right\}_{n \in \mathbb{N}} \subset \mathcal{U}_{\text {ad }}$ with the corresponding sequence of states $\left\{\left(\mu_{n}, \varphi_{n}, \sigma_{n}\right)\right\}_{n \in \mathbb{N}}$ to have

$$
\lim _{n \rightarrow \infty} \mathcal{J}\left(\left(\mu_{n}, \varphi_{n}, \sigma_{n}\right), \mathbf{u}_{n}\right)=\inf _{\mathbf{v} \in \mathcal{U}_{\mathrm{ad}}} \mathcal{J}(\mathcal{S}(\mathbf{v}), \mathbf{v}) \geq 0,
$$

where $\mathcal{S}(\mathbf{v})$ denotes the state corresponding to the control $\mathbf{v}$. Furthermore, by combining the estimates (2.38)(2.40), which are uniform with respect to $n$, with the structure of $\mathcal{U}_{\mathrm{ad}}$, it is a standard matter (upon extracting a subsequence that we do not relabel) to infer the existence of limits $\overline{\mathbf{u}} \in \mathcal{U}_{\text {ad }}$ and $\bar{\mu}, \bar{\varphi}, \bar{\sigma}$ such that, as $n \rightarrow \infty$,

$$
\begin{aligned}
& \mathbf{u}_{n} \rightarrow \overline{\mathbf{u}} \quad \text { weakly star in } L^{\infty}(Q)^{2}, \\
& \mu_{n} \rightarrow \bar{\mu} \quad \text { weakly star in } H^{1}(0, T ; H) \cap L^{\infty}(0, T ; V) \cap L^{2}\left(0, T ; W_{0}\right) \cap L^{\infty}(Q), \\
& \varphi_{n} \rightarrow \bar{\varphi} \quad \text { weakly star in } W^{1, \infty}(0, T ; H) \cap H^{1}(0, T ; V) \cap L^{\infty}\left(0, T ; W_{0}\right) \cap L^{\infty}(Q), \\
& \sigma_{n} \rightarrow \bar{\sigma} \quad \text { weakly star in } H^{1}(0, T ; H) \cap L^{\infty}(0, T ; V) \cap L^{2}\left(0, T ; W_{0}\right) \cap L^{\infty}(Q),
\end{aligned}
$$

and, by the compactness of the embedding $W_{0} \subset C^{0}(\bar{\Omega})$, also that (see, e.g., [44], Sect. 8, Cor. 4),

$$
\varphi_{n} \rightarrow \bar{\varphi} \quad \text { strongly in } C^{0}(\bar{Q})
$$

It is then a standard matter to pass to the limit as $n \rightarrow \infty$ in the formulation (1.2)-(1.6) written for $\left(\mu_{n}, \varphi_{n}, \sigma_{n}\right)$ and $\mathbf{u}_{n}$ to conclude that $(\bar{\mu}, \bar{\varphi}, \bar{\sigma})=\mathcal{S}(\overline{\mathbf{u}})$, and then to exploit the weak lower semicontinuity of $\mathcal{J}$ to derive that $\overline{\mathbf{u}}$ is a minimizer for the optimization problem (eP). 


\section{DifFERENTIABILITY PROPERTIES OF THE SOLUTION OPERATOR}

We now discuss the Fréchet differentiability of $\mathcal{S}$, considered as a mapping between suitable Banach spaces. To show such a result, it is favorable to employ the implicit function theorem, because, if applicable, it yields that the control-to-state operator automatically inherits the differentiability order from that of the involved nonlinearities. The technique employed here is an adaptation of that used recently in [45] in a similar context. For the reader's convenience, we give the details of the argument. For this, some functional analytic preparations are in order. We first define the linear spaces

$$
\begin{aligned}
X & :=X \times \widetilde{X} \times X, \quad \text { where } \\
X & :=H^{1}(0, T ; H) \cap C^{0}([0, T] ; V) \cap L^{2}\left(0, T ; W_{0}\right) \cap L^{\infty}(Q), \\
\widetilde{X} & :=W^{1, \infty}(0, T ; H) \cap H^{1}(0, T ; V) \cap L^{\infty}\left(0, T ; W_{0}\right) \cap C^{0}(\bar{Q}),
\end{aligned}
$$

which are Banach spaces when endowed with their natural norms. Next, we introduce the linear space

$$
\begin{gathered}
y:=\left\{(\mu, \varphi, \sigma) \in X: \alpha \partial_{t} \mu+\partial_{t} \varphi-\Delta \mu \in L^{\infty}(Q), \beta \partial_{t} \varphi-\Delta \varphi-\mu \in L^{\infty}(Q),\right. \\
\left.\partial_{t} \sigma-\Delta \sigma+\chi \Delta \varphi \in L^{\infty}(Q)\right\}
\end{gathered}
$$

which becomes a Banach space when endowed with the norm

$$
\begin{aligned}
\|(\mu, \varphi, \sigma)\|_{y}:= & \|(\mu, \varphi, \sigma)\| x+\left\|\alpha \partial_{t} \mu+\partial_{t} \varphi-\Delta \mu\right\|_{L^{\infty}(Q)}+\left\|\beta \partial_{t} \varphi-\Delta \varphi-\mu\right\|_{L^{\infty}(Q)} \\
& +\left\|\partial_{t} \sigma-\Delta \sigma+\chi \Delta \varphi\right\|_{L^{\infty}(Q)} .
\end{aligned}
$$

Finally, we fix constants $\tilde{r}_{-}, \tilde{r}_{+}$such that

$$
r_{-}<\tilde{r}_{-}<r_{*} \leq r^{*}<\tilde{r}_{+}<r_{+},
$$

with the constants introduced in (S2) and (2.39). We then consider the set

$$
\Phi:=\left\{(\mu, \varphi, \sigma) \in y: \tilde{r}_{-}<\varphi(x, t)<\tilde{r}_{+} \text {for all }(x, t) \in \bar{Q}\right\}
$$

which is obviously an open subset of the space $y$.

We first prove an auxiliary result for the linear initial-boundary value problem

$$
\begin{aligned}
& \alpha \partial_{t} \mu+\partial_{t} \varphi-\Delta \mu=\lambda_{1}\left[P(\bar{\varphi})(\sigma-\chi \varphi-\mu)+P^{\prime}(\bar{\varphi})(\bar{\sigma}+\chi(1-\bar{\varphi})-\bar{\mu}) \varphi-\mathbb{h}^{\prime}(\bar{\varphi}) \bar{u}_{1} \varphi\right] \\
& -\lambda_{2} \mathfrak{h}(\bar{\varphi}) h_{1}+\lambda_{3} f_{1} \text { in } Q \text {, } \\
& \beta \partial_{t} \varphi-\Delta \varphi-\mu=\lambda_{1}\left[\chi \sigma-F^{\prime \prime}(\bar{\varphi}) \varphi\right]+\lambda_{3} f_{2} \text { in } Q \text {, } \\
& \partial_{t} \sigma-\Delta \sigma+\chi \Delta \varphi=\lambda_{1}\left[-P(\bar{\varphi})(\sigma-\chi \varphi-\mu)-P^{\prime}(\bar{\varphi})(\bar{\sigma}+\chi(1-\bar{\varphi})-\bar{\mu}) \varphi\right] \\
& +\lambda_{2} h_{2}+\lambda_{3} f_{3} \text { in } Q \\
& \partial_{\mathbf{n}} \mu=\partial_{\mathbf{n}} \varphi=\partial_{\mathbf{n}} \sigma=0 \quad \text { on } \Sigma \text {, } \\
& \mu(0)=\lambda_{4} \mu_{0}, \quad \varphi(0)=\lambda_{4} \varphi_{0}, \quad \sigma(0)=\lambda_{4} \sigma_{0}, \quad \text { in } \Omega,
\end{aligned}
$$

which for $\lambda_{1}=\lambda_{2}=1$ and $\lambda_{3}=\lambda_{4}=0$ coincides with the linearization of the state equation at $\left(\left(\bar{u}_{1}, \bar{u}_{2}\right),(\bar{\mu}, \bar{\varphi}, \bar{\sigma})\right)$. We will need this slightly more general version later for the application of the implicit function theorem. To shorten the exposition, we introduce the Banach space of the initial data satisfying (2.33), 
which is given by

$$
\mathcal{N}:=\left\{\left(\mu_{0}, \varphi_{0}, \sigma_{0}\right): \mu_{0}, \sigma_{0} \in H^{1}(\Omega) \cap L^{\infty}(\Omega), \varphi_{0} \in W_{0}\right\}
$$

endowed with its natural norm.

Lemma 4.1. Suppose that $\lambda_{1}, \lambda_{2}, \lambda_{3}, \lambda_{4} \in\{0,1\}$ are given and that the assumptions (W1), (S1)(S4), and (2.32)-(2.34) are fulfilled. Moreover, let $\left(\left(\bar{u}_{1}, \bar{u}_{2}\right),(\bar{\mu}, \bar{\varphi}, \bar{\sigma})\right) \in \mathcal{U}_{R} \times \Phi$ be arbitrary. Then the linear initial-boundary value problem (4.6)-(4.10) has for every $\left(h_{1}, h_{2}\right) \in L^{\infty}(Q)^{2}$ and $\left(f_{1}, f_{2}, f_{3}\right) \in$ $L^{\infty}(Q) \times\left(H^{1}(0, T ; H) \cap L^{\infty}(Q)\right) \times L^{\infty}(Q)$ a unique solution $(\mu, \varphi, \sigma) \in \mathcal{y}$. Moreover, the linear mapping

$$
\left(\left(h_{1}, h_{2}\right),\left(f_{1}, f_{2}, f_{3}\right),\left(\mu_{0}, \varphi_{0}, \sigma_{0}\right)\right) \mapsto(\mu, \varphi, \sigma)
$$

is continuous from $L^{\infty}(Q)^{2} \times\left(L^{\infty}(Q) \times\left(H^{1}(0, T ; H) \cap L^{\infty}(Q)\right) \times L^{\infty}(Q)\right) \times \mathcal{N}$ into $y$.

Proof. We use a standard Faedo-Galerkin approximation. To this end, let $\left\{\lambda_{k}\right\}_{k \in \mathbb{N}}$ and $\left\{e_{k}\right\}_{k \in \mathbb{N}}$ denote the eigenvalues and associated eigenfunctions of the eigenvalue problem

$$
-\Delta y+y=\lambda y \quad \text { in } \Omega, \quad \partial_{\mathbf{n}} y=0 \quad \text { on } \Gamma,
$$

where the latter are normalized by $\left\|e_{k}\right\|=1$ for all $k \in \mathbb{N}$. Then $\left\{e_{k}\right\}_{k \in \mathbb{N}}$ forms a complete orthonormal system in $H$ which is also dense in $V$. We put $V_{n}:=\operatorname{span}\left\{e_{1}, \ldots, e_{n}\right\}, n \in \mathbb{N}$, noting that $\bigcup_{n \in \mathbb{N}} V_{n}$ is dense in $V$. We look for functions of the form

$$
\mu_{n}(x, t)=\sum_{k=1}^{n} \rho_{k}^{(n)}(t) e_{k}(x), \quad \varphi_{n}(x, t)=\sum_{k=1}^{n} v_{k}^{(n)}(t) e_{k}(x), \quad \sigma_{n}(x, t)=\sum_{k=1}^{n} w_{k}^{(n)}(t) e_{k}(x),
$$

that satisfy the system

$$
\begin{aligned}
& \left(\alpha \partial_{t} \mu_{n}(t), v\right)+\left(\partial_{t} \varphi_{n}(t), v\right)+\left(\nabla \mu_{n}(t), \nabla v\right)=\left(z_{n 1}(t), v\right), \\
& \left(\beta \partial_{t} \varphi_{n}(t), v\right)+\left(\nabla \varphi_{n}(t), \nabla v\right)-\left(\mu_{n}(t), v\right)=\left(z_{n 2}(t), v\right), \\
& \left(\partial_{t} \sigma_{n}(t), v\right)+\left(\nabla \sigma_{n}(t), \nabla v\right)-\chi\left(\nabla \varphi_{n}(t), \nabla v\right)=\left(z_{n 3}(t), v\right), \\
& \mu_{n}(0)=\lambda_{4} \mathbb{P}_{n} \mu_{0}, \quad \varphi_{n}(0)=\lambda_{4} \mathbb{P}_{n} \varphi_{0}, \quad \sigma_{n}(0)=\lambda_{4} \mathbb{P}_{n} \sigma_{0},
\end{aligned}
$$

for all $v \in V_{n}$, and almost every $t \in(0, T)$, where $\mathbb{P}_{n}$ denotes the $H^{1}(\Omega)$-orthogonal projection onto $V_{n}$, and where

$$
\begin{aligned}
z_{n 1}= & \lambda_{1}\left[P(\bar{\varphi})\left(\sigma_{n}-\chi \varphi_{n}-\mu_{n}\right)+P^{\prime}(\bar{\varphi})(\bar{\sigma}+\chi(1-\bar{\varphi})-\bar{\mu}) \varphi_{n}-\mathbb{h}^{\prime}(\bar{\varphi}) \bar{u}_{1} \varphi_{n}\right] \\
& -\lambda_{2} \mathfrak{h}(\bar{\varphi}) h_{1}+\lambda_{3} f_{1} \\
z_{n 2}= & \lambda_{1}\left[\chi \sigma_{n}-F^{\prime \prime}(\bar{\varphi}) \varphi_{n}\right]+\lambda_{3} f_{2} \\
z_{n 3}= & \lambda_{1}\left[-P(\bar{\varphi})\left(\sigma_{n}-\chi \varphi_{n}-\mu_{n}\right)-P^{\prime}(\bar{\varphi})(\bar{\sigma}+\chi(1-\bar{\varphi})-\bar{\mu}) \varphi_{n}\right] \\
& +\lambda_{2} h_{2}+\lambda_{3} f_{3} .
\end{aligned}
$$

Insertion of $v=e_{k}$, for $k \in \mathbb{N}$, in (4.12)-(4.14), and substitution for the second summand in (4.12) by means of (4.13), then lead to an initial value problem for an explicit linear system of ordinary differential equations for the unknowns $\rho_{1}^{(n)}, \ldots, \rho_{n}^{(n)}, v_{1}^{(n)}, \ldots, v_{n}^{(n)}, w_{1}^{(n)}, \ldots, w_{n}^{(n)}$, in which all of the coefficient functions belong to $L^{\infty}(0, T)$. Hence, by virtue of Carathéodory's theorem, there exists a unique solution in $W^{1, \infty}\left(0, T ; \mathbb{R}^{3 n}\right)$ that specifies the unique solution $\left(\mu_{n}, \varphi_{n}, \sigma_{n}\right) \in W^{1, \infty}\left(0, T ; W_{0}\right)^{3}$ to the system (4.12)-(4.15), for $n \in \mathbb{N}$. 
We now derive some a priori estimates for the Faedo-Galerkin approximations. In this procedure, $C_{i}>0$, $i \in \mathbb{N}$, will denote constants that are independent of $n \in \mathbb{N}$ and the data $\left(\left(f_{1}, f_{2}, f_{3}\right),\left(\mu_{0}, \varphi_{0}, \sigma_{0}\right)\right)$, while the constant $M>0$ is given by

$$
\begin{aligned}
M:= & \lambda_{2}\left\|\left(h_{1}, h_{2}\right)\right\|_{L^{\infty}(Q)^{2}}+\lambda_{3}\left\|\left(f_{1}, f_{2}, f_{3}\right)\right\|_{L^{\infty}(Q) \times\left(H^{1}(0, T ; H) \cap L^{\infty}(Q)\right) \times L^{\infty}(Q)} \\
& +\lambda_{4}\left\|\left(\mu_{0}, \varphi_{0}, \sigma_{0}\right)\right\|_{\mathcal{N}} .
\end{aligned}
$$

Moreover, $(\bar{\mu}, \bar{\varphi}, \bar{\sigma}) \in \Phi$, and thus it follows that $\bar{\mu}, \bar{\sigma} \in L^{\infty}(Q)$ and $\bar{\varphi}, \mathfrak{h}(\bar{\varphi}), \mathbb{h}^{\prime}(\bar{\varphi}), P(\bar{\varphi}), P^{\prime}(\bar{\varphi}), F^{\prime \prime}(\bar{\varphi}) \in C^{0}(\bar{Q})$. Hence, there is some constant $C_{1}>0$ such that, for a.e. $(x, t) \in Q$ and for all $n \in \mathbb{N}$,

$$
\begin{aligned}
\left(\left|z_{n 1}\right|+\left|z_{n 2}\right|+\left|z_{n 3}\right|\right)(x, t) \leq & C_{1}\left(\lambda_{1}\left(\left|\mu_{n}\right|+\left|\varphi_{n}\right|+\left|\sigma_{n}\right|\right)(x, t)+\lambda_{2}\left(\left|h_{1}\right|+\left|h_{2}\right|\right)(x, t)\right. \\
& \left.+\lambda_{3}\left(\left|f_{1}\right|+\left|f_{2}\right|+\left|f_{3}\right|\right)(x, t)\right) \\
\leq & C_{1}\left(\lambda_{1}\left(\left|\mu_{n}\right|+\left|\varphi_{n}\right|+\left|\sigma_{n}\right|\right)(x, t)+M\right)
\end{aligned}
$$

First estimate: We insert $v=\mu_{n}(t)$ in (4.12), $v=\partial_{t} \varphi_{n}(t)$ in (4.13), $v=\sigma_{n}(t)$ in (4.14) and add the resulting equations, whence a cancellation of two terms occurs. Then, we add to both sides of the resulting equation the same term $\frac{1}{2} \frac{\mathrm{d}}{\mathrm{d} t}\left\|\varphi_{n}(t)\right\|^{2}=\left(\varphi_{n}(t), \partial_{t} \varphi_{n}(t)\right)$. Integration over $[0, \tau]$, where $\tau \in(0, T]$, then yields the identity

$$
\begin{aligned}
\frac{1}{2}\left(\alpha\left\|\mu_{n}(\tau)\right\|^{2}+\left\|\varphi_{n}(\tau)\right\|_{V}^{2}+\left\|\sigma_{n}(\tau)\right\|^{2}\right)+\int_{Q_{\tau}}\left(\left|\nabla \mu_{n}\right|^{2}+\left|\nabla \sigma_{n}\right|^{2}\right)+\beta \int_{Q_{\tau}}\left|\partial_{t} \varphi_{n}\right|^{2} \\
=\frac{\lambda_{4}^{2}}{2}\left(\alpha\left\|\mathbb{P}_{n} \mu_{0}\right\|^{2}+\left\|\mathbb{P}_{n} \varphi_{0}\right\|_{V}^{2}+\left\|\mathbb{P}_{n} \sigma_{0}\right\|^{2}\right)+\int_{0}^{\tau}\left(\mu_{n}(t), z_{n 1}(t)\right) \mathrm{d} t+\int_{0}^{\tau}\left(z_{n 3}(t), \sigma_{n}(t)\right) \mathrm{d} t \\
\quad+\int_{0}^{\tau}\left(z_{n 2}(t)+\varphi_{n}(t), \partial_{t} \varphi_{n}(t)\right) \mathrm{d} t+\chi \int_{0}^{\tau}\left(\nabla \varphi_{n}(t), \nabla \sigma_{n}(t)\right) \mathrm{d} t=: \sum_{i=1}^{5} I_{i}
\end{aligned}
$$

with obvious notation. We estimate the terms on the right-hand side individually. First observe that, for all $n \in \mathbb{N}$,

$$
I_{1} \leq C_{2} \lambda_{4}^{2}\left\|\left(\mathbb{P}_{n} \mu_{0}, \mathbb{P}_{n} \varphi_{0}, \mathbb{P}_{n} \sigma_{0}\right)\right\|_{V \times V \times V}^{2} \leq C_{2} \lambda_{4}^{2}\left\|\left(\mu_{0}, \varphi_{0}, \sigma_{0}\right)\right\|_{V \times V \times V}^{2} \leq C_{2} M^{2}
$$

Moreover, by virtue of (4.20) and Young's inequality, we deduce that

$$
I_{2}+I_{3} \leq C_{3} M^{2}+C_{4} \int_{Q_{\tau}}\left(\left|\mu_{n}\right|^{2}+\left|\varphi_{n}\right|^{2}+\left|\sigma_{n}\right|^{2}\right) .
$$

Likewise, it results that

$$
I_{4} \leq \frac{\beta}{2} \int_{Q_{\tau}}\left|\partial_{t} \varphi_{n}\right|^{2}+\frac{C_{5}}{\beta} M^{2}+\frac{C_{6}}{\beta} \int_{Q_{\tau}}\left(\left|\mu_{n}\right|^{2}+\left|\varphi_{n}\right|^{2}+\left|\sigma_{n}\right|^{2}\right)
$$

Finally, we have that

$$
I_{5} \leq \frac{\chi^{2}}{2} \int_{Q_{\tau}}\left|\nabla \varphi_{n}\right|^{2}+\frac{1}{2} \int_{Q_{\tau}}\left|\nabla \sigma_{n}\right|^{2}
$$


Combining the estimates (4.21)-(4.25), we have shown that

$$
\begin{aligned}
& \frac{1}{2}\left(\alpha\left\|\mu_{n}(\tau)\right\|^{2}+\left\|\varphi_{n}(\tau)\right\|_{V}^{2}+\left\|\sigma_{n}(\tau)\right\|^{2}\right)+\int_{Q_{\tau}}\left(\left|\nabla \mu_{n}\right|^{2}+\frac{1}{2}\left|\nabla \sigma_{n}\right|^{2}\right)+\frac{\beta}{2} \int_{Q_{\tau}}\left|\partial_{t} \varphi_{n}\right|^{2} \\
& \quad \leq C_{7} M^{2}+C_{8} \int_{0}^{\tau}\left(\left\|\mu_{n}(t)\right\|^{2}+\left\|\varphi_{n}(t)\right\|_{V}^{2}+\left\|\sigma_{n}(t)\right\|^{2}\right) \mathrm{d} t
\end{aligned}
$$

Therefore, invoking Gronwall's lemma, we conclude that

$$
\begin{gathered}
\left\|\mu_{n}\right\|_{L^{\infty}(0, T ; H) \cap L^{2}(0, T ; V)}+\left\|\varphi_{n}\right\|_{H^{1}(0, T ; H) \cap L^{\infty}(0, T ; V)} \\
+\left\|\sigma_{n}\right\|_{L^{\infty}(0, T ; H) \cap L^{2}(0, T ; V)} \leq C_{9} M \quad \forall n \in \mathbb{N} .
\end{gathered}
$$

SeCond EStimate: Next, we insert $v=\partial_{t} \mu_{n}(t)$ in $(4.12)$ and integrate over $[0, \tau]$, where $\tau \in(0, T]$, to obtain the identity

$$
\begin{aligned}
\frac{1}{2} & \left\|\nabla \mu_{n}(\tau)\right\|^{2}+\alpha \int_{0}^{\tau}\left\|\partial_{t} \mu_{n}(t)\right\|^{2} \mathrm{~d} t \\
& =\frac{\lambda_{4}^{2}}{2}\left\|\nabla \mathbb{P}_{n} \mu_{0}\right\|^{2}+\int_{0}^{\tau}\left(z_{n 1}(t), \partial_{t} \mu_{n}(t)\right) \mathrm{d} t-\int_{0}^{\tau}\left(\partial_{t} \varphi_{n}(t), \partial_{t} \mu_{n}(t)\right) \mathrm{d} t .
\end{aligned}
$$

Applying Young's inequality appropriately and making use of (4.20) and (4.26), we conclude the estimate

$$
\left\|\mu_{n}\right\|_{H^{1}(0, T ; H) \cap L^{\infty}(0, T ; V)} \leq C_{10} M \quad \forall n \in \mathbb{N} .
$$

Third estimate: At this point, we insert $v=-\Delta \mu_{n}(t)$ in (4.12) and $v=-\Delta \varphi_{n}(t)$ in (4.13), add, and integrate over $[0, \tau]$, where $\tau \in(0, T]$. We then obtain that

$$
\begin{aligned}
\frac{\alpha}{2} \| & \nabla \mu_{n}(\tau)\left\|^{2}+\frac{\beta}{2}\right\| \nabla \varphi_{n}(\tau)\left\|^{2}+\int_{0}^{\tau}\right\| \Delta \mu_{n}(t)\left\|^{2} \mathrm{~d} t+\int_{0}^{\tau}\right\| \Delta \varphi_{n}(t) \|^{2} \mathrm{~d} t \\
= & \frac{\alpha \lambda_{4}^{2}}{2}\left\|\nabla \mathbb{P}_{n} \mu_{0}\right\|^{2}+\frac{\beta \lambda_{4}^{2}}{2}\left\|\nabla \mathbb{P}_{n} \varphi_{0}\right\|^{2}-\int_{0}^{\tau}\left(z_{n 1}(t)-\partial_{t} \varphi_{n}(t), \Delta \mu_{n}(t)\right) \mathrm{d} t \\
& -\int_{0}^{\tau}\left(\mu_{n}(t)+z_{n 2}(t), \Delta \varphi_{n}(t)\right) \mathrm{d} t
\end{aligned}
$$

whence, using (4.20), (4.26), (4.27) and Young's inequality,

$$
\int_{0}^{T}\left(\left\|\Delta \mu_{n}(t)\right\|^{2}+\left\|\Delta \varphi_{n}(t)\right\|^{2}\right) \mathrm{d} t \leq C_{11} M^{2} \quad \forall n \in \mathbb{N}
$$

Classical elliptic estimates, using (4.9) and (4.26)-(4.28), then yield that

$$
\left\|\mu_{n}\right\|_{L^{2}\left(0, T ; H^{2}(\Omega)\right)}+\left\|\varphi_{n}\right\|_{L^{2}\left(0, T ; H^{2}(\Omega)\right)} \leq C_{12} M \quad \forall n \in \mathbb{N} .
$$

With the estimate (4.29) at hand, we may (by first taking $v=\partial_{t} \sigma_{n}(t)$ in (4.14) and then $v=-\Delta \sigma_{n}(t)$ ) infer by similar reasoning that also

$$
\left\|\sigma_{n}\right\|_{H^{1}(0, T ; H) \cap L^{\infty}(0, T ; V) \cap L^{2}\left(0, T ; H^{2}(\Omega)\right)} \leq C_{13} M \quad \forall n \in \mathbb{N} .
$$


At this point, we can conclude from standard weak and weak star compactness arguments the existence of a triple $(\mu, \varphi, \sigma)$ such that, possibly only on a subsequence which is again indexed by $n$,

$$
\begin{aligned}
& \mu_{n} \rightarrow \mu, \quad \varphi_{n} \rightarrow \varphi, \quad \sigma_{n} \rightarrow \sigma, \\
& \text { all weakly star in } H^{1}(0, T ; H) \cap L^{\infty}(0, T ; V) \cap L^{2}\left(0, T ; W_{0}\right) .
\end{aligned}
$$

Standard arguments, which need no repetition here, then show that $(\mu, \varphi, \sigma)$ is a strong solution to the system (4.6)-(4.10). In particular, it turns out that $\partial_{\mathbf{n}} \mu=\partial_{\mathbf{n}} \varphi=\partial_{\mathbf{n}} \sigma=0$ almost everywhere on $\Sigma$. Moreover, recalling (4.26)-(4.30), and invoking the weak sequential lower semicontinuity of norms, we conclude that there is some $C_{14}>0$ such that

$$
\|(\mu, \varphi, \sigma)\|_{z} \leq C_{14} M
$$

where $z$ is defined in $(2.2)$.

We now derive further estimates for $(\mu, \varphi, \sigma)$. In the next one we argue formally, noting that it can be carried out rigorously on the level of the Faedo-Galerkin approximations. Indeed, we differentiate (4.7) formally with respect to time to obtain the identity

$$
\beta \partial_{t}\left(\partial_{t} \varphi\right)-\Delta \partial_{t} \varphi=\partial_{t} \mu+\lambda_{1}\left(\chi \partial_{t} \sigma-F^{\prime \prime \prime}(\bar{\varphi}) \partial_{t} \bar{\varphi} \varphi-F^{\prime \prime}(\bar{\varphi}) \partial_{t} \varphi\right)+\lambda_{3} \partial_{t} f_{2}=: g_{\varphi}
$$

Testing (4.32) formally by $\partial_{t} \varphi$ and integrating formally by parts, we find that

$$
\frac{\beta}{2}\left\|\partial_{t} \varphi(t)\right\|^{2}+\int_{Q_{t}}\left|\nabla \partial_{t} \varphi\right|^{2}=\frac{\beta}{2}\left\|\partial_{t} \varphi(0)\right\|^{2}+\int_{Q_{t}} g_{\varphi} \partial_{t} \varphi
$$

Now observe that, owing to (4.31), (2.38), (2.40), and (4.19) we have that

$$
\int_{Q_{t}} g_{\varphi} \partial_{t} \varphi \leq\left\|g_{\varphi}\right\|_{L^{2}(0, T ; H)}\left\|\partial_{t} \varphi\right\|_{L^{2}(0, T ; H)} \leq C_{15} M^{2}
$$

As for (4.34), we point out that the term $-\lambda_{1} F^{\prime \prime \prime}(\bar{\varphi}) \partial_{t} \bar{\varphi} \varphi$, which is part of $g_{\varphi}$, is bounded in $L^{2}(0, T ; H)$ since $V \subset L^{4}(\Omega)$ with continuous embedding, and consequently it follows that $\left\|\partial_{t} \bar{\varphi}\right\|_{L^{2}\left(0, T ; L^{4}(\Omega)\right)}$ is under control and $\|\varphi\|_{L^{\infty}\left(0, T ; L^{4}(\Omega)\right)} \leq C_{16} M$, whence

$$
\begin{aligned}
& \left\|-\lambda_{1} F^{\prime \prime \prime}(\bar{\varphi}) \partial_{t} \bar{\varphi} \varphi\right\|_{L^{2}(0, T ; H)} \\
& \quad \leq \lambda_{1}\left\|F^{\prime \prime \prime}(\bar{\varphi})\right\|_{L^{\infty}(Q)}\left\|\partial_{t} \bar{\varphi}\right\|_{L^{2}\left(0, T ; L^{4}(\Omega)\right)}\|\varphi\|_{L^{\infty}\left(0, T ; L^{4}(\Omega)\right)} \leq C_{17} M .
\end{aligned}
$$

Next, writing (4.7) for $t=0$ and recalling (4.10), we have that

$$
\partial_{t} \varphi(0)=\beta^{-1}\left(\lambda_{4}\left(\Delta \varphi_{0}+\mu_{0}+\lambda_{1} \chi \sigma_{0}-\lambda_{1} F^{\prime \prime}\left(\varphi_{0}\right) \varphi_{0}\right)+\lambda_{3} f_{2}(0)\right),
$$

and it follows from $(2.33),(2.34),(4.19)$ that

$$
\left\|\partial_{t} \varphi(0)\right\| \leq C_{18} M
$$

Combining (4.33)-(4.35), and invoking Young's inequality and Gronwall's lemma, we thus can conclude that

$$
\|\varphi\|_{W^{1, \infty}(0, T ; H) \cap H^{1}(0, T ; V)} \leq C_{19} M .
$$


Now, in view of (4.36) and (4.31), a comparison of terms in (4.7) and standard elliptic estimates yield that

$$
\|\varphi\|_{L^{\infty}\left(0, T ; H^{2}(\Omega)\right)} \leq C_{20} M
$$

and the compactness of the embedding $\left(W^{1, \infty}(0, T ; H) \cap L^{\infty}\left(0, T ; H^{2}(\Omega)\right)\right) \subset C^{0}(\bar{Q})$ (see, e.g., [44], Sect. 8, Cor. 4) then shows that also

$$
\|\varphi\|_{C^{0}(\bar{Q})} \leq C_{21} M
$$

At this point, we observe that, by bringing the term $\partial_{t} \varphi$ to the right-hand side, equation (4.6) can be rewritten as a linear parabolic equation for $\mu$ whose right-hand side is already known to be bounded in $L^{\infty}(0, T ; H)$ by an expression of the form $C_{22} M$. Since $\mu$ satisfies zero Neumann boundary conditions and $\mu_{0} \in H^{1}(\Omega) \cap L^{\infty}(\Omega)$, we can apply the classical result of Theorem 7.1 in [36] to conclude that $\mu \in L^{\infty}(Q)$ and

$$
\|\mu\|_{L^{\infty}(Q)} \leq C_{23} M
$$

Similar reasoning on equation (4.8), invoking the $L^{\infty}(0, T ; H)$-bound for $\Delta \varphi$ implied by (4.37), shows that also $\sigma \in L^{\infty}(Q)$ and

$$
\|\sigma\|_{L^{\infty}(Q)} \leq C_{24} M
$$

About the linear dependence of the right-hand sides of (4.39) and (4.40) on the constant $M$ that is specified in (4.19), we point out that this dependence is a consequence of the linearity of the problem (4.6)-(4.10). Indeed, e.g., if we choose a full set of data for which $M=1$ and prove the above estimates, then we obtain all of the bounds (4.31) and (4.36)-(4.40) with some particular constants - fully determined - and without specification of $M$. Next, we can take a generic set of data for which the constant $M(\neq 0)$ in $(4.19)$ is different from 1 . Thus, pick the corresponding solution $(\mu, \varphi, \sigma)$ of (4.6)-(4.10) and divide all components of the triplet $(\mu, \varphi, \sigma)$ by $M$; then, the scaled triplet $(\mu / M, \varphi / M, \sigma / M)$ solves another problem in which the data are all divided by $M$ and satisfy (4.19) with constant 1 . Hence, the previously found universal estimates work also for $(\mu / M, \varphi / M, \sigma / M)$ with the same constants as before. As a consequence, it is straightforward to finally obtain (4.39) and (4.40) (and previous estimates as well), simply by multiplying by $M$.

At this point, we can combine all the estimates (4.31), (4.36)-(4.40) and deduce that

$$
\|(\mu, \varphi, \sigma)\|_{x} \leq C_{25} M
$$

$X$ being defined in (4.1). Moreover, it is readily seen from the equations (4.6)-(4.8) that $(\mu, \varphi, \sigma) \in \mathcal{y}(c f .(4.2))$ and that

$$
\|(\mu, \varphi, \sigma)\|_{y} \leq C_{26} M
$$

The existence of a solution with the asserted properties is thus shown. It remains to prove the uniqueness. To this end, let $\left(\mu_{i}, \varphi_{i}, \sigma_{i}\right) \in \mathcal{y}, i=1,2$, be two solutions to the system. Then $(\mu, \varphi, \sigma):=\left(\mu_{1}, \varphi_{1}, \sigma_{1}\right)-\left(\mu_{2}, \varphi_{2}, \sigma_{2}\right)$ solves the system (4.6)-(4.10) with zero initial data, where the terms $\lambda_{2} h_{i}, i=1,2$, and $\lambda_{3} f_{i}, i=1,2,3$, on the right-hand sides do not occur. By the definition of $y$ (recall (4.1) and (4.2)), and since $(\mu, \varphi, \sigma) \in \mathcal{y}$, all of the generalized partial derivatives occurring in (4.6)-(4.8) belong to $L^{2}(Q)$. Therefore, we may repeat - now for the continuous problem - the a priori estimates performed for the Faedo-Galerkin approximations that led us to the estimate (4.26). We then find analogous estimates for $(\mu, \varphi, \sigma)$, where this time the constant $M$ from (4.19) equals zero. Thus, $(\mu, \varphi, \sigma)=(0,0,0)$. With this, the uniqueness is shown, which finishes the proof of the assertion. 
Remark 4.2. A careful inspection of the estimates (4.21)-(4.30) shows that the term $M$ appearing on the right-hand side of the corresponding estimates can be replaced by

$$
\widetilde{M}:=\lambda_{2}\left\|\left(h_{1}, h_{2}\right)\right\|_{L^{2}(0, T ; H)^{2}}+\lambda_{3}\left\|\left(f_{1}, f_{2}, f_{3}\right)\right\|_{L^{2}(0, T ; H)^{3}}+\lambda_{4}\left\|\left(\mu_{0}, \varphi_{0}, \sigma_{0}\right)\right\|_{V^{3}},
$$

since, in particular, only the $L^{2}(Q)$ norms of the increments $h_{i}, i=1,2$, and of the source terms $f_{i}, i=1,2,3$, enter the computations. This, along with (4.31), entails that the linearized variables $(\mu, \varphi, \sigma)$ (which correspond to the choices $\left.\lambda_{1}=\lambda_{2}=1, \lambda_{3}=\lambda_{4}=0\right)$ satisfy

$$
\|(\mu, \varphi, \sigma)\|_{z} \leq c\|\mathbf{h}\|_{L^{2}(0, T ; H)^{2}}
$$

with some positive constant $c$ ( $c f$. also Rem. 4.5), being $\mathbf{h}=\left(h_{1}, h_{2}\right)$.

Having proved Lemma 4.1, we are in a position to prepare for the application of the implicit function theorem. For this purpose, let us consider two auxiliary linear initial-boundary value problems. The first,

$$
\begin{array}{ll}
\alpha \partial_{t} \mu+\partial_{t} \varphi-\Delta \mu=f_{1} & \text { in } Q, \\
\beta \partial_{t} \varphi-\Delta \varphi-\mu=f_{2} & \text { in } Q, \\
\partial_{t} \sigma-\Delta \sigma+\chi \Delta \varphi=f_{3} & \text { in } Q, \\
\partial_{\mathbf{n}} \mu=\partial_{\mathbf{n}} \varphi=\partial_{\mathbf{n}} \sigma=0 & \text { on } \Sigma, \\
\mu(0)=\varphi(0)=\sigma(0)=0 & \text { in } \Omega,
\end{array}
$$

is obtained from (4.6)-(4.10) for $\lambda_{1}=\lambda_{2}=\lambda_{4}=0, \lambda_{3}=1$. Thanks to Lemma 4.1, this system has for each $\left(f_{1}, f_{2}, f_{3}\right) \in L^{\infty}(Q) \times\left(H^{1}(0, T ; H) \cap L^{\infty}(Q)\right) \times L^{\infty}(Q)$ a unique solution $(\mu, \varphi, \sigma) \in \mathcal{y}$, and the associated linear mapping

$$
\mathcal{G}_{1}:\left(L^{\infty}(Q) \times\left(H^{1}(0, T ; H) \cap L^{\infty}(Q)\right) \times L^{\infty}(Q)\right) \rightarrow \mathfrak{y} ;\left(f_{1}, f_{2}, f_{3}\right) \mapsto(\mu, \varphi, \sigma),
$$

is continuous. The second system reads

$$
\begin{array}{ll}
\alpha \partial_{t} \mu+\partial_{t} \varphi-\Delta \mu=0 & \text { in } Q, \\
\beta \partial_{t} \varphi-\Delta \varphi-\mu=0 & \text { in } Q, \\
\partial_{t} \sigma-\Delta \sigma+\chi \Delta \varphi=0 & \text { in } Q, \\
\partial_{\mathbf{n}} \mu=\partial_{\mathbf{n}} \varphi=\partial_{\mathbf{n}} \sigma=0 & \text { on } \Sigma, \\
\mu(0)=\mu_{0}, \quad \varphi(0)=\varphi_{0}, \quad \sigma(0)=\sigma_{0} & \text { in } \Omega,
\end{array}
$$

and results from (4.6)-(4.10) for $\lambda_{1}=\lambda_{2}=\lambda_{3}=0, \lambda_{4}=1$. For each $\left(\mu_{0}, \varphi_{0}, \sigma_{0}\right) \in \mathcal{N}$, it also enjoys a unique solution $(\mu, \varphi, \sigma) \in \mathcal{y}$, and the associated mapping

$$
\mathcal{G}_{2}: \mathcal{N} \rightarrow \mathrm{y} ;\left(\mu_{0}, \varphi_{0}, \sigma_{0}\right) \mapsto(\mu, \varphi, \sigma),
$$

is linear and continuous as well. In addition, we define on the open set $\mathcal{A}:=\left(\mathcal{U}_{R} \times \Phi\right) \subset(\mathcal{U} \times \mathcal{y})$ the nonlinear mapping

$$
\begin{aligned}
& \mathcal{G}_{3}: \mathcal{A} \rightarrow\left(L^{\infty}(Q) \times\left(H^{1}(0, T ; H) \cap L^{\infty}(Q)\right) \times L^{\infty}(Q)\right) ; \\
& \left(\left(u_{1}, u_{2}\right),(\mu, \varphi, \sigma)\right) \mapsto\left(f_{1}, f_{2}, f_{3}\right), \text { where }
\end{aligned}
$$




$$
\begin{gathered}
\left(f_{1}, f_{2}, f_{3}\right)=\left(P(\varphi)(\sigma+\chi(1-\varphi)-\mu)-\mathbb{h}(\varphi) u_{1}, \chi \sigma-F^{\prime}(\varphi),\right. \\
\left.-P(\varphi)(\sigma+\chi(1-\varphi)-\mu)+u_{2}\right) .
\end{gathered}
$$

The solution $(\mu, \varphi, \sigma)$ to the nonlinear state equation (1.2)-(1.6) is the sum of the solution to the system (4.44)(4.48), where $\left(f_{1}, f_{2}, f_{3}\right)$ is chosen as above (with $(\mu, \varphi, \sigma)$ considered as known), and of the solution to the system (4.50)-(4.54). Therefore, the state vector $(\mu, \varphi, \sigma)$ associated with the control vector $\left(u_{1}, u_{2}\right)$ is the unique solution to the nonlinear equation

$$
(\mu, \varphi, \sigma)=\mathcal{G}_{1}\left(\mathcal{G}_{3}\left(\left(u_{1}, u_{2}\right),(\mu, \varphi, \sigma)\right)+\mathcal{G}_{2}\left(\mu_{0}, \varphi_{0}, \sigma_{0}\right) .\right.
$$

Let us now define the nonlinear mapping $\mathcal{F}: \mathcal{A} \rightarrow y$,

$$
\mathcal{F}\left(\left(u_{1}, u_{2}\right),(\mu, \varphi, \sigma)\right)=\mathcal{G}_{1}\left(\mathcal{G}_{3}\left(\left(u_{1}, u_{2}\right),(\mu, \varphi, \sigma)\right)+\mathcal{G}_{2}\left(\mu_{0}, \varphi_{0}, \sigma_{0}\right)-(\mu, \varphi, \sigma) .\right.
$$

With $\mathcal{F}$, the state equation can be shortly written as

$$
\mathcal{F}\left(\left(u_{1}, u_{2}\right),(\mu, \varphi, \sigma)\right)=(0,0,0) .
$$

This equation just means that $(\mu, \varphi, \sigma)$ is a solution to the state system $(1.2)-(1.6)$ such that $\left(\left(u_{1}, u_{2}\right),(\mu, \varphi, \sigma)\right) \in$ $\mathcal{A}$. From Theorem 2.3 we know that such a solution exists for every $\left(u_{1}, u_{2}\right) \in \mathcal{U}_{R}$. A fortiori, any such solution automatically enjoys the separation property (2.39) and is uniquely determined.

We are going to apply the implicit function theorem to the equation (4.59). To this end, we need the differentiability of the involved mappings.

Observe that, owing to the differentiability properties of the involved Nemytskii operators (see, e.g., [47], Thm. 4.22, p. 229), the mapping $\mathcal{G}_{3}$ is twice continuously Fréchet differentiable into the space $L^{\infty}(Q) \times L^{\infty}(Q) \times$ $L^{\infty}(Q)$, and for the first partial derivatives at any point $\left(\left(\bar{u}_{1}, \bar{u}_{2}\right),(\bar{\mu}, \bar{\varphi}, \bar{\sigma})\right) \in \mathcal{A}$, and for all $\left(u_{1}, u_{2}\right) \in \mathcal{U}$ and $(\mu, \varphi, \sigma) \in \mathcal{y}$, we have the identities

$$
\begin{aligned}
& D_{\left(u_{1}, u_{2}\right)} \mathcal{G}_{3}\left(\left(\bar{u}_{1}, \bar{u}_{2}\right),(\bar{\mu}, \bar{\varphi}, \bar{\sigma})\right)\left(u_{1}, u_{2}\right)=\left(-\mathbb{h}(\bar{\varphi}) u_{1}, 0, u_{2}\right) \\
& D_{(\mu, \varphi, \sigma)} \mathcal{G}_{3}\left(\left(\bar{u}_{1}, \bar{u}_{2}\right),(\bar{\mu}, \bar{\varphi}, \bar{\sigma})\right)(\mu, \varphi, \sigma) \\
& =\left(P(\bar{\varphi})(\sigma-\chi \varphi-\mu)+P^{\prime}(\bar{\varphi})(\bar{\sigma}+\chi(1-\bar{\varphi})-\bar{\mu}) \varphi-\mathbb{h}^{\prime}(\bar{\varphi}) \bar{u}_{1} \varphi, \chi_{\sigma}-F^{\prime \prime}(\bar{\varphi}) \varphi\right. \\
& \left.\quad-P(\bar{\varphi})\left(\sigma-\chi_{\varphi}-\mu\right)-P^{\prime}(\bar{\varphi})(\bar{\sigma}+\chi(1-\bar{\varphi})-\bar{\mu}) \varphi\right) .
\end{aligned}
$$

It remains to show that the second component of $\mathcal{G}_{3}$ is also twice continuously Fréchet differentiable into the space $H^{1}(0, T ; H)$. But this follows exactly as in ([45], Sect. 2, (2.70) ff.). We thus can refer the reader to [45] for this argument, just pointing out that the regularity requirement $F \in C^{5}\left(r_{-}, r_{+}\right)$in (S2) is crucial for this argument.

At this point, we may apply the chain rule, which yields that $\mathcal{F}$ is twice continuously Fréchet differentiable from $\mathcal{U}_{R} \times \Phi$ into $y$, with the first-order partial derivatives

$$
\begin{aligned}
& D_{\left(u_{1}, u_{2}\right)} \mathcal{F}\left(\left(\bar{u}_{1}, \bar{u}_{2}\right),(\bar{\mu}, \bar{\varphi}, \bar{\sigma})\right)=\mathcal{G}_{1} \circ D_{\left(u_{1}, u_{2}\right)} \mathcal{G}_{3}\left(\left(\bar{u}_{1}, \bar{u}_{2}\right),(\bar{\mu}, \bar{\varphi}, \bar{\sigma})\right), \\
& D_{(\mu, \varphi, \sigma)} \mathcal{F}\left(\left(\bar{u}_{1}, \bar{u}_{2}\right),(\bar{\mu}, \bar{\varphi}, \bar{\sigma})\right)=\mathcal{G}_{1} \circ D_{(\mu, \varphi, \sigma)} \mathcal{G}_{3}\left(\left(\bar{u}_{1}, \bar{u}_{2}\right),(\bar{\mu}, \bar{\varphi}, \bar{\sigma})\right)-I_{y},
\end{aligned}
$$

where $I_{y}$ denotes the identity mapping on $y$.

At this point, we introduce for convenience abbreviating denotations, namely,

$$
\mathbf{u}:=\left(u_{1}, u_{2}\right), \quad \overline{\mathbf{u}}:=\left(\bar{u}_{1}, \bar{u}_{2}\right), \quad \mathbf{y}:=(\mu, \varphi, \sigma), \quad \overline{\mathbf{y}}:=(\bar{\mu}, \bar{\varphi}, \bar{\sigma}),
$$




$$
\mathbf{y}_{0}:=\left(\mu_{0}, \varphi_{0}, \sigma_{0}\right), \quad \mathbf{0}:=(0,0,0)
$$

With these denotations, we want to prove the differentiability of the control-to-state mapping $\mathbf{u} \mapsto \mathbf{y}$ defined implicitly by the equation $\mathcal{F}(\mathbf{u}, \mathbf{y})=\mathbf{0}$, using the implicit function theorem. Now let $\overline{\mathbf{u}} \in \mathcal{U}_{R}$ be given and $\overline{\mathbf{y}}=\mathcal{S}(\overline{\mathbf{u}})$. We need to show that the linear and continuous operator $D_{\mathbf{y}} \mathcal{F}(\overline{\mathbf{u}}, \overline{\mathbf{y}})$ is a topological isomorphism from $y$ into itself.

To this end, let $\mathbf{v} \in \mathbf{y}$ be arbitrary. Then the identity $D_{\mathbf{y}} \mathcal{F}(\overline{\mathbf{u}}, \overline{\mathbf{y}})(\mathbf{y})=\mathbf{v}$ just means that $\mathcal{G}_{1}\left(D_{\mathbf{y}} \mathcal{G}_{3}(\overline{\mathbf{u}}, \overline{\mathbf{y}})(\mathbf{y})\right)-\mathbf{y}=\mathbf{v}$, which is equivalent to saying that

$$
\mathbf{w}:=\mathbf{y}+\mathbf{v}=\mathcal{G}_{1}\left(D_{\mathbf{y}} \mathcal{G}_{3}(\overline{\mathbf{u}}, \overline{\mathbf{y}})(\mathbf{w})\right)-\mathcal{G}_{1}\left(D_{\mathbf{y}} \mathcal{G}_{3}(\overline{\mathbf{u}}, \overline{\mathbf{y}})(\mathbf{v})\right)
$$

The latter identity means that $\mathbf{w}$ is a solution to (4.6)-(4.10) for $\lambda_{1}=\lambda_{3}=1, \lambda_{2}=\lambda_{4}=0$, with the specification $\left(f_{1}, f_{2}, f_{3}\right)=-\mathcal{G}_{1}\left(D_{\mathbf{y}} \mathcal{G}_{3}(\overline{\mathbf{u}}, \overline{\mathbf{y}})(\mathbf{v})\right) \in \mathbf{y}$. By Lemma 4.1, such a solution $\mathbf{w} \in \boldsymbol{y}$ exists and is uniquely determined. We thus can infer that $D_{\mathbf{y}} \mathcal{F}(\overline{\mathbf{u}}, \overline{\mathbf{y}})$ is surjective. At the same time, taking $\mathbf{v}=\mathbf{0}$, we see that the equation $D_{\mathbf{y}} \mathcal{F}(\overline{\mathbf{u}}, \overline{\mathbf{y}})(\mathbf{y})=\mathbf{0}$ means that $\mathbf{y}$ is the unique solution to (4.6)-(4.10) for $\lambda_{1}=1, \lambda_{2}=\lambda_{3}=\lambda_{4}=0$. Obviously, $\mathbf{y}=\mathbf{0}$, which implies that $D_{\mathbf{y}} \mathcal{F}(\overline{\mathbf{u}}, \overline{\mathbf{y}})$ is also injective and thus, by the open mapping principle, a topological isomorphism from $y$ into itself.

At this point, we may employ the implicit function theorem (cf., e.g., [3], Thms. 4.7.1 and 5.4.5 or [16], 10.2.1) to conclude that the mapping $\mathcal{S}$ is twice continuously Fréchet differentiable from $\mathcal{U}_{R}$ into $y$ and that the first Fréchet derivative $D \mathcal{S}(\overline{\mathbf{u}})$ of $\mathcal{S}$ at $\overline{\mathbf{u}} \in \mathcal{U}_{R}$ is given by the formula

$$
D S(\overline{\mathbf{u}})=-D_{\mathbf{y}} \mathcal{F}(\overline{\mathbf{u}}, \overline{\mathbf{y}})^{-1} \circ D_{\mathbf{u}} \mathcal{F}(\overline{\mathbf{u}}, \overline{\mathbf{y}}) .
$$

Now let $\mathbf{h}=\left(h_{1}, h_{2}\right) \in \mathcal{U}$ be arbitrary and $\mathbf{y}=(\mu, \varphi, \sigma)=D \mathcal{S}(\overline{\mathbf{u}})(\mathbf{h})$. Then,

$$
D_{\mathbf{y}} \mathcal{F}(\overline{\mathbf{u}}, \overline{\mathbf{y}})(\mathbf{y})=-D_{\mathbf{u}} \mathcal{F}(\overline{\mathbf{u}}, \overline{\mathbf{y}})(\mathbf{h}),
$$

which is obviously equivalent to saying that

$$
\mathbf{y}=\mathcal{G}_{1}\left(D_{\mathbf{y}} \mathcal{G}_{3}(\overline{\mathbf{u}}, \overline{\mathbf{y}})(\mathbf{y})\right)+\mathcal{G}_{1}\left(-\mathbb{h}(\bar{\varphi}) h_{1}, 0, h_{2}\right) .
$$

This, in turn, means that $\mathbf{y}$ is the unique solution to the problem (4.6)-(4.10) for $\lambda_{1}=\lambda_{2}=1, \lambda_{3}=\lambda_{4}=0$.

In summary, we have shown the following result.

Theorem 4.3 (Fréchet differentiability of $\mathcal{S}$ ). Suppose that the conditions (W1), (S1)-(S4), and (2.32) are fulfilled. Moreover, let the initial data $\left(\mu_{0}, \varphi_{0}, \sigma_{0}\right)$ verify (2.33) and (2.34), and let $\overline{\mathbf{u}}=\left(\bar{u}_{1}, \bar{u}_{2}\right) \in \mathcal{U}_{R}$ be arbitrary and $(\bar{\mu}, \bar{\varphi}, \bar{\sigma})=\mathcal{S}(\overline{\mathbf{u}})$. Then the control-to-state operator $\mathcal{S}$ is twice continuously Fréchet differentiable at $\overline{\mathbf{u}}$ as a mapping from $\mathfrak{U}$ into $\mathfrak{y}$. Moreover, for every $\mathbf{h}=\left(h_{1}, h_{2}\right) \in \mathcal{U}$, the Fréchet derivative $D \mathcal{S}(\overline{\mathbf{u}}) \in \mathcal{L}(\mathcal{U}, \mathcal{y})$ of $\mathcal{S}$ at $\overline{\mathbf{u}}$ is given by the identity $D \mathcal{S}(\overline{\mathbf{u}})(\mathbf{h})=(\mu, \varphi, \sigma)$, where $(\mu, \varphi, \sigma)$ is the unique solution to the linear system (4.6)-(4.10) with $\lambda_{1}=\lambda_{2}=1, \lambda_{3}=\lambda_{4}=0$.

Motivated by the forthcoming analysis, we now present a stability estimate for the solutions to the linearized system. In abuse of notation, we will denote the linearized variable associated with $\varphi$ by $\xi$, which up to now was devoted to indicate a selection of the subdifferential $\partial F_{1}$ evaluated at some point. Since the optimal control problem demands to work with strong solutions, we no longer have any selection to work with, so that from now on the variable $\xi$ will play a different role $(c f$. Thm. 2.3). We have the following result, where we recall the definition $(2.3)$ of the space $\mathcal{V}$.

Theorem 4.4. Suppose that the conditions (W1), (S1)-(S4), (C3) and (2.32) are fulfilled. Moreover, let the initial data $\left(\mu_{0}, \varphi_{0}, \sigma_{0}\right)$ satisfy $(2.33)-(2.34)$, and let $\overline{\mathbf{u}}^{i}=\left(\bar{u}_{1}^{i}, \bar{u}_{2}^{i}\right) \in \mathcal{U}_{R}$ be arbitrary and $\left(\bar{\mu}_{i}, \bar{\varphi}_{i}, \bar{\sigma}_{i}\right)=\mathcal{S}\left(\overline{\mathbf{u}}^{i}\right), i=$ 
1,2. Furthermore, let $\left(\eta_{i}, \xi_{i}, \theta_{i}\right)$ denote the associated solutions to the linearized system (i.e., the system (4.6)(4.10) with $\lambda_{1}=\lambda_{2}=1, \lambda_{3}=\lambda_{4}=0$ ). Then the mapping DS is Lipschitz continuous on $\mathcal{U}_{R}$ in the sense that there exists a positive constant $K_{d}$ such that for all $\mathbf{h} \in \mathcal{U}$ we have

$$
\left\|\left(D \mathcal{S}\left(\overline{\mathbf{u}}^{1}\right)-D \mathcal{S}\left(\overline{\mathbf{u}}^{2}\right)\right)(\mathbf{h})\right\|_{\mathcal{v}} \leq K_{d}\left\|\overline{\mathbf{u}}^{1}-\overline{\mathbf{u}}^{2}\right\|_{L^{2}(0, T ; H)^{2}}\|\mathbf{h}\|_{L^{2}(0, T ; H)^{2}}
$$

Proof. Due to Theorem 4.3, the proof of (4.65) reduces to showing that there exists a constant $c>0$ such that

$$
\left\|\left(\eta_{1}, \xi_{1}, \theta_{1}\right)-\left(\eta_{2}, \xi_{2}, \theta_{2}\right)\right\|_{\mathcal{V}} \leq c\left\|\overline{\mathbf{u}}^{1}-\overline{\mathbf{u}}^{2}\right\|_{L^{2}(0, T ; H)^{2}}\|\mathbf{h}\|_{L^{2}(0, T ; H)^{2}}
$$

which is the estimate we are going to check. Moreover, let us notice that (4.43) in Remark 4.2 guarantees the existence of a positive constant $c$ such that

$$
\left\|\left(\eta_{i}, \xi_{i}, \theta_{i}\right)\right\|_{z} \leq c\|\mathbf{h}\|_{L^{2}(0, T ; H)^{2}}, \quad i=1,2
$$

Next, we set

$$
\begin{aligned}
& \bar{\mu}=\bar{\mu}_{1}-\bar{\mu}_{2}, \quad \bar{\varphi}=\bar{\varphi}_{1}-\bar{\varphi}_{2}, \quad \bar{\sigma}=\bar{\sigma}_{1}-\bar{\sigma}_{2}, \\
& \eta=\eta_{1}-\eta_{2}, \quad \xi=\xi_{1}-\xi_{2}, \quad \theta=\theta_{1}-\theta_{2},
\end{aligned}
$$

and observe that the triple $(\eta, \xi, \theta)$ solves the system obtained from taking the difference between the linearized system written for $\left(\eta_{1}, \xi_{1}, \theta_{1}\right)$ and $\left(\eta_{2}, \xi_{2}, \theta_{2}\right)$, which reads

$$
\begin{array}{ll}
\alpha \partial_{t} \eta+\partial_{t} \xi-\Delta \eta=f_{1}+f_{2} & \text { in } Q, \\
\beta \partial_{t} \xi-\Delta \xi+\left(F^{\prime \prime}\left(\bar{\varphi}_{1}\right)-F^{\prime \prime}\left(\bar{\varphi}_{2}\right)\right) \xi_{1}+F^{\prime \prime}\left(\bar{\varphi}_{2}\right) \xi=\eta+\chi \theta & \text { in } Q, \\
\partial_{t} \theta-\Delta \theta+\chi \Delta \xi=-f_{1} & \text { in } Q, \\
\partial_{\mathbf{n}} \eta=\partial_{\mathbf{n}} \xi=\partial_{\mathbf{n}} \theta=0 & \text { on } \Sigma, \\
\eta(0)=\xi(0)=\theta(0)=0 & \text { in } \Omega,
\end{array}
$$

where now $f_{1}$ and $f_{2}$ are specified by

$$
\begin{aligned}
f_{1}= & \left(P\left(\bar{\varphi}_{1}\right)-P\left(\bar{\varphi}_{2}\right)\right)\left(\theta_{1}-\chi \xi_{1}-\eta_{1}\right)+P\left(\bar{\varphi}_{2}\right)(\theta-\chi \xi-\eta) \\
& +\left(P^{\prime}\left(\bar{\varphi}_{1}\right)-P^{\prime}\left(\bar{\varphi}_{2}\right)\right) \xi_{1}\left(\bar{\sigma}_{1}+\chi\left(1-\bar{\varphi}_{1}\right)-\bar{\mu}_{1}\right)+P^{\prime}\left(\bar{\varphi}_{2}\right) \xi\left(\bar{\sigma}_{1}+\chi\left(1-\bar{\varphi}_{1}\right)-\bar{\mu}_{1}\right) \\
& +P^{\prime}\left(\bar{\varphi}_{2}\right) \xi_{2}(\bar{\sigma}-\chi \bar{\varphi}-\bar{\mu}), \\
f_{2}= & -\left(\mathbb{h}^{\prime}\left(\bar{\varphi}_{1}\right)-\mathbb{h}^{\prime}\left(\bar{\varphi}_{2}\right)\right) \xi_{1} \bar{u}_{1}^{1}-\mathbb{h}^{\prime}\left(\bar{\varphi}_{2}\right) \xi \bar{u}_{1}^{1}-\mathbb{h}^{\prime}\left(\bar{\varphi}_{2}\right) \xi_{2}\left(\bar{u}_{1}^{1}-\bar{u}_{1}^{2}\right)-\left(\mathbb{h}_{(}\left(\bar{\varphi}_{1}\right)-\mathbb{h}\left(\bar{\varphi}_{2}\right)\right) h_{1} .
\end{aligned}
$$

Moreover, let us recall that due to (2.41), we have the stability estimate

$$
\left\|\bar{\mu}_{1}-\bar{\mu}_{2}\right\|_{Z}+\left\|\bar{\varphi}_{1}-\bar{\varphi}_{2}\right\|_{Z}+\left\|\bar{\sigma}_{1}-\bar{\sigma}_{2}\right\|_{Z} \leq c\left\|\overline{\mathbf{u}}^{1}-\overline{\mathbf{u}}^{2}\right\|_{L^{2}(0, T ; H)^{2}}
$$

We now aim at deriving some a priori estimates for the differences $(\eta, \xi, \theta)$ which will entail (4.66). Prior to this, let us premise a general fact that will be employed several times later on. To this end, let $f: \mathbb{R} \rightarrow \mathbb{R}$ be a regular, bounded and Lipschitz continuous function with Lipschitz continuous and bounded derivative $f^{\prime}$ (in what follows the role of $f$ will be played by $P, \mathbb{h}, F$, and possibly their derivatives). Let $\varphi_{1}$ and $\varphi_{2}$ be the second components of different strong solutions $\left(\mu_{1}, \varphi_{1}, \sigma_{1}\right)$ and $\left(\mu_{2}, \varphi_{2}, \sigma_{2}\right)$ associated with controls $\mathbf{u}^{1}, \mathbf{u}^{2} \in \mathcal{U}_{R}$ according to Theorem 2.3. Then it follows from the continuity of the embeddings $V \subset L^{p}(\Omega)$ and 
$W_{0} \subset W^{1, p}(\Omega), p \in[1,6]$, and the estimate $(2.38)$ that, a. e. in $(0, T)$,

$$
\begin{aligned}
\left\|f\left(\varphi_{1}\right)-f\left(\varphi_{2}\right)\right\|_{p} & \leq c\left\|f\left(\varphi_{1}\right)-f\left(\varphi_{2}\right)\right\|_{V} \\
& =c\left\|f\left(\varphi_{1}\right)-f\left(\varphi_{2}\right)\right\|+c\left\|f^{\prime}\left(\varphi_{1}\right) \nabla \varphi_{1}-f^{\prime}\left(\varphi_{2}\right) \nabla \varphi_{2}\right\| \\
& \leq c\left\|\varphi_{1}-\varphi_{2}\right\|+c\left\|\left(f^{\prime}\left(\varphi_{1}\right)-f^{\prime}\left(\varphi_{2}\right)\right) \nabla \varphi_{1}+f^{\prime}\left(\varphi_{2}\right) \nabla\left(\varphi_{1}-\varphi_{2}\right)\right\| \\
& \leq c\left(\left\|\varphi_{1}-\varphi_{2}\right\|+\left\|\varphi_{1}-\varphi_{2}\right\|_{4}\left\|\nabla \varphi_{1}\right\|_{4}+\left\|\nabla\left(\varphi_{1}-\varphi_{2}\right)\right\|\right) \\
& \leq c\left(1+K_{1}\right)\left\|\varphi_{1}-\varphi_{2}\right\|_{V} .
\end{aligned}
$$

In addition, for the sake of a shorter exposition, in the upcoming estimates we will avoid to explicitly write the integration variable $s$ in the time integrals.

First estimate: We test (4.68) by $\eta,(4.69)$, to which we add $\xi$ on both sides, by $\partial_{t} \xi$, and (4.70) by $\theta$, add the resulting equalities and integrate over time, obtaining

$$
\begin{aligned}
& \frac{\alpha}{2}\|\eta(t)\|^{2}+\int_{Q_{t}}|\nabla \eta|^{2}+\beta \int_{Q_{t}}\left|\partial_{t} \xi\right|^{2}+\frac{1}{2}\|\xi(t)\|_{V}^{2}+\frac{1}{2}\|\theta(t)\|^{2}+\int_{Q_{t}}|\nabla \theta|^{2} \\
& =\int_{Q_{t}} f_{1}(\eta-\theta)+\int_{Q_{t}} f_{2} \eta-\int_{Q_{t}}\left(F^{\prime \prime}\left(\bar{\varphi}_{1}\right)-F^{\prime \prime}\left(\bar{\varphi}_{2}\right)\right) \xi_{1} \partial_{t} \xi-\int_{Q_{t}} F^{\prime \prime}\left(\bar{\varphi}_{2}\right) \xi \partial_{t} \xi \\
& \quad+\int_{Q_{t}}(\eta+\chi \theta+\xi) \partial_{t} \xi+\chi \int_{Q_{t}} \nabla \xi \cdot \nabla \theta=: I_{1}+\ldots+I_{6} .
\end{aligned}
$$

Using the Young and Hölder inequalities, the Lipschitz continuity of $P, P^{\prime}$ and $\mathbb{h}^{\prime}$, the continuous embedding $V \subset L^{4}(\Omega)$, the uniform bounds (2.38)-(2.40) for $\left(\bar{\mu}_{i}, \bar{\varphi}_{i}, \bar{\sigma}_{i}\right), i=1,2$, as well as (4.67), (4.73), and (4.74), we infer that

$$
\begin{aligned}
I_{1} \leq & c \int_{Q_{t}}\left(|\eta|^{2}+|\xi|^{2}+|\theta|^{2}\right)+c \int_{0}^{t}\left\|P\left(\bar{\varphi}_{1}\right)-P\left(\bar{\varphi}_{2}\right)\right\|_{4}^{2}\left(\left\|\theta_{1}\right\|_{4}^{2}+\left\|\xi_{1}\right\|_{4}^{2}+\left\|\eta_{1}\right\|_{4}^{2}\right) \mathrm{d} s \\
& +c\left(\left\|\bar{\sigma}_{1}\right\|_{L^{\infty}(Q)}^{2}+\left\|\bar{\varphi}_{1}\right\|_{L^{\infty}(Q)}^{2}+\left\|\bar{\mu}_{1}\right\|_{L^{\infty}(Q)}^{2}+1\right) \int_{0}^{t}\left\|P^{\prime}\left(\bar{\varphi}_{1}\right)-P^{\prime}\left(\bar{\varphi}_{2}\right)\right\|_{4}^{2}\left\|\xi_{1}\right\|_{4}^{2} \mathrm{~d} s \\
& +c\left(\left\|\bar{\sigma}_{1}\right\|_{L^{\infty}(Q)}^{2}+\left\|\bar{\varphi}_{1}\right\|_{L^{\infty}(Q)}^{2}+\left\|\bar{\mu}_{1}\right\|_{L^{\infty}(Q)}^{2}+1\right) \int_{Q_{t}}|\xi|^{2} \\
& +c \int_{0}^{t}\left(\left\|\bar{\sigma}_{1}-\bar{\sigma}_{2}\right\|_{4}^{2}+\left\|\bar{\varphi}_{1}-\bar{\varphi}_{2}\right\|_{4}^{2}+\left\|\bar{\mu}_{1}-\bar{\mu}_{2}\right\|_{4}^{2}\right)\left\|\xi_{2}\right\|_{4}^{2} \mathrm{~d} s \\
\leq & c \int_{Q_{t}}\left(|\eta|^{2}+|\xi|^{2}+|\theta|^{2}\right) \\
& +c\left(\left\|\theta_{1}\right\|_{L^{\infty}(0, T ; V)}^{2}+\left\|\xi_{1}\right\|_{L^{\infty}(0, T ; V)}^{2}+\left\|\eta_{1}\right\|_{L^{\infty}(0, T ; V)}^{2}\right) \int_{0}^{t}\left\|\bar{\varphi}_{1}-\bar{\varphi}_{2}\right\|_{V}^{2} \mathrm{~d} s \\
& +c\left\|\xi_{1}\right\|_{L^{\infty}(0, T ; V)}^{2}\left(\left\|\bar{\sigma}_{1}\right\|_{L^{\infty}(Q)}^{2}+\left\|\bar{\varphi}_{1}\right\|_{L^{\infty}(Q)}^{2}+\left\|\bar{\mu}_{1}\right\|_{L^{\infty}(Q)}^{2}+1\right) \int_{0}^{t}\left\|\bar{\varphi}_{1}-\bar{\varphi}_{2}\right\|_{V}^{2} \mathrm{~d} s \\
& +c\left(\left\|\bar{\sigma}_{1}\right\|_{L^{\infty}(Q)}^{2}+\left\|\bar{\varphi}_{1}\right\|_{L^{\infty}(Q)}^{2}+\left\|\bar{\mu}_{1}\right\|_{L^{\infty}(Q)}^{2}+1\right) \int_{Q_{t}}|\xi|^{2} \\
& +c\left\|\xi_{2}\right\|_{L^{\infty}(0, T ; V)}^{2} \int_{0}^{t}\left(\left\|\bar{\sigma}_{1}-\bar{\sigma}_{2}\right\|_{V}^{2}+\left\|\bar{\varphi}_{1}-\bar{\varphi}_{2}\right\|_{V}^{2}+\left\|\bar{\mu}_{1}-\bar{\mu}_{2}\right\|_{V}^{2}\right) \mathrm{d} s \\
\leq & c \int_{Q_{t}}\left(|\eta|^{2}+|\xi|^{2}+|\theta|^{2}\right)+c\left\|\overline{\mathbf{u}}^{1}-\overline{\mathbf{u}}^{2}\right\|_{L^{2}(0, T ; H)^{2}}^{2}\|\mathbf{h}\|_{L^{2}(0, T ; H)^{2} .}^{2} .
\end{aligned}
$$


Next, by similar computations, we deduce that, for any $\delta>0$ (to be chosen later),

$$
\begin{aligned}
I_{2} \leq & c \int_{Q_{t}}|\eta|^{2}+c\left\|\bar{u}_{1}^{1}\right\|_{L^{\infty}(Q)}^{2} \int_{0}^{t}\left\|\mathbb{h}^{\prime}\left(\bar{\varphi}_{1}\right)-\mathbb{h}^{\prime}\left(\bar{\varphi}_{2}\right)\right\|_{4}^{2}\left\|\xi_{1}\right\|_{4}^{2} \mathrm{~d} s+c\left\|_{1}^{1}\right\|_{L^{\infty}(Q)}^{2} \int_{Q_{t}}|\xi|^{2} \\
& +2 \delta \int_{0}^{t}\|\eta\|_{V}^{2} \mathrm{~d} s+c_{\delta} \int_{0}^{t}\left\|\overline{\mathbf{u}}^{1}-\overline{\mathbf{u}}^{2}\right\|^{2}\left\|\xi_{2}\right\|_{4}^{2} \mathrm{~d} s+c_{\delta} \int_{0}^{t}\left\|\mathfrak{h}\left(\bar{\varphi}_{1}\right)-\mathbb{h}\left(\bar{\varphi}_{2}\right)\right\|_{4}^{2}\left\|h_{1}\right\|^{2} \mathrm{~d} s \\
\leq & (c+2 \delta) \int_{Q_{t}}|\eta|^{2}+c\left\|\xi_{1}\right\|_{L^{\infty}(0, T ; V)}^{2}\left\|\bar{u}_{1}^{1}\right\|_{L^{\infty}(Q)}^{2} \int_{0}^{t}\left\|\bar{\varphi}_{1}-\bar{\varphi}_{2}\right\|_{V}^{2} \mathrm{~d} s \\
& +c\left\|\bar{u}_{1}^{1}\right\|_{L^{\infty}(Q)}^{2} \int_{Q_{t}}|\xi|^{2}+2 \delta \int_{Q_{t}}|\nabla \eta|^{2} \\
& +c_{\delta}\left\|\xi_{2}\right\|_{L^{\infty}(0, T ; V)}^{2} \int_{0}^{t}\left\|\overline{\mathbf{u}}^{1}-\overline{\mathbf{u}}^{2}\right\|^{2} \mathrm{~d} s+c_{\delta} \int_{0}^{t}\left\|\bar{\varphi}_{1}-\bar{\varphi}_{2}\right\|_{V}^{2}\left\|h_{1}\right\|^{2} \mathrm{~d} s \\
\leq & 2 \delta \int_{Q_{t}}|\nabla \eta|^{2}+c_{\delta} \int_{Q_{t}}|\eta|^{2}+c \int_{Q_{t}}|\xi|^{2}+c_{\delta}\left\|\overline{\mathbf{u}}^{1}-\overline{\mathbf{u}}^{2}\right\|_{L^{2}(0, T ; H)^{2}}^{2}\|\mathbf{h}\|_{L^{2}(0, T ; H)^{2}}^{2}
\end{aligned}
$$

The terms involving the potentials can be easily handled by invoking the separation principle (2.39), which entails the Lipschitz continuity of $F$ and of its derivatives. Using this, (4.67), (4.74), and the Young inequality, we obtain that

$$
\begin{aligned}
I_{3}+I_{4} & \leq \delta \int_{Q_{t}}\left|\partial_{t} \xi\right|^{2}+c_{\delta}\left\|\xi_{1}\right\|_{L^{\infty}(0, T ; V)}^{2} \int_{0}^{t}\left\|\bar{\varphi}_{1}-\bar{\varphi}_{2}\right\|_{V}^{2} \mathrm{~d} s+c_{\delta} \int_{Q_{t}}|\xi|^{2} \\
& \leq \delta \int_{Q_{t}}\left|\partial_{t} \xi\right|^{2}+c_{\delta}\left\|\overline{\mathbf{u}}^{1}-\overline{\mathbf{u}}^{2}\right\|_{L^{2}(0, T ; H)^{2}}^{2}\|\mathbf{h}\|_{L^{2}(0, T ; H)^{2}}^{2}+c_{\delta} \int_{Q_{t}}|\xi|^{2} .
\end{aligned}
$$

Finally, we have that

$$
I_{5}+I_{6} \leq \delta \int_{Q_{t}}\left(\left|\partial_{t} \xi\right|^{2}+|\nabla \theta|^{2}\right)+c_{\delta} \int_{Q_{t}}\left(|\eta|^{2}+|\theta|^{2}+|\xi|^{2}+|\nabla \xi|^{2}\right)
$$

At this point, we collect the above estimates and adjust $\delta \in(0,1)$ small enough. Gronwall's lemma then yields that

$$
\begin{aligned}
& \|\eta\|_{L^{\infty}(0, T ; H) \cap L^{2}(0, T ; V)}+\|\xi\|_{H^{1}(0, T ; H) \cap L^{\infty}(0, T ; V)}+\|\theta\|_{L^{\infty}(0, T ; H) \cap L^{2}(0, T ; V)} \\
& \quad \leq c\left\|\overline{\mathbf{u}}^{1}-\overline{\mathbf{u}}^{2}\right\|_{L^{2}(0, T ; H)^{2}}\|\mathbf{h}\|_{L^{2}(0, T ; H)^{2}} .
\end{aligned}
$$

Second estimate: Estimate (4.75) entails that the term $\partial_{t} \xi$ is bounded in $L^{2}(0, T ; H)$ by the expression on the right-hand side of the above estimate. Hence, equation (4.69) can be expressed as an elliptic equation for $\xi$ whose right-hand side is bounded in $L^{2}(0, T ; H)$ by the same expression. Therefore, we easily get from elliptic regularity theory that

$$
\|\xi\|_{L^{2}\left(0, T ; W_{0}\right)} \leq c\left\|\overline{\mathbf{u}}^{1}-\overline{\mathbf{u}}^{2}\right\|_{L^{2}(0, T ; H)^{2}}\|\mathbf{h}\|_{L^{2}(0, T ; H)^{2}} .
$$

This concludes the proof of Theorem 4.4.

Remark 4.5. Let us point out that Theorem 4.3 establishes the Fréchet differentiability of $\mathcal{S}$ as a mapping from $L^{\infty}(Q)^{2}$ into $\mathcal{y}$, a space of very regular functions. However, the Fréchet differentiability can also be directly obtained as a mapping from $L^{2}(0, T ; H)^{2}$ into a space of less regular functions. Indeed, a closer look at the proof 
of Theorem 2.5 in [39] reveals that the line of argumentation employed there can straightforwardly be adapted to our present situation, yielding that in the notation used there it holds that

$$
\left\|\left(\bar{\mu}^{\mathbf{h}}-\bar{\mu}-\eta^{\mathbf{h}}, \bar{\varphi}^{\mathbf{h}}-\bar{\varphi}-\xi^{\mathbf{h}}, \bar{\sigma}^{\mathbf{h}}-\bar{\sigma}-\theta^{\mathbf{h}}\right)\right\|_{z} \leq c\|\mathbf{h}\|_{L^{2}(0, T ; H)^{2}}^{2},
$$

which in turn entails that the control-to-state operator $\mathcal{S}$ is Fréchet differentiable as a mapping from $L^{2}(0, T ; H)^{2}$ into $z \supset y$. We have chosen to not follow this approach because, although it will suffice to handle the firstorder necessary conditions pointed out below in Section 5, it would not allow us to deal with the second-order sufficient conditions established in Section 6.

Motivated by the forthcoming analysis on the second-order sufficient optimality conditions, let us now also explicitly identify the second-order Fréchet derivative of the control-to-state operator $\mathcal{S}$, which exists according to Theorem 4.3. To this end, let $\overline{\mathbf{u}} \in \mathcal{U}_{R}$ be given. For arbitrary increments $\mathbf{h}, \mathbf{k} \in \mathcal{U}$, we set

$$
(\bar{\mu}, \bar{\varphi}, \bar{\sigma}):=\mathcal{S}(\overline{\mathbf{u}}), \quad\left(\eta^{\mathbf{h}}, \xi^{\mathbf{h}}, \theta^{\mathbf{h}}\right):=D \mathcal{S}(\overline{\mathbf{u}})(\mathbf{h}), \quad\left(\eta^{\mathbf{k}}, \xi^{\mathbf{k}}, \theta^{\mathbf{k}}\right):=D \mathcal{S}(\overline{\mathbf{u}})(\mathbf{k})
$$

where it is known that $\left(\eta^{\mathbf{h}}, \xi^{\mathbf{h}}, \theta^{\mathbf{h}}\right),\left(\eta^{\mathbf{k}}, \xi^{\mathbf{k}}, \theta^{\mathbf{k}}\right) \in \boldsymbol{y}$. Now, if one adapts the argumentation of the proof of Theorem 5.16, pp. 288-289 in [47] to the present situation, starting from the identities (4.57)-(4.63), then one concludes that the second-order Fréchet derivative $D^{2} \mathcal{S}(\overline{\mathbf{u}})(\mathbf{k})(\mathbf{h})$ can be evaluated using the system (4.78)-(4.82) introduced below. Since we intend to give an independent proof, we do not give the details of the argument, here. We begin our analysis with the following result.

Theorem 4.6. Assume that (W1), (S1)-(S4), (2.32)-(2.34) are fulfilled. Then the following initial-boundary value problem, which will be referred to as the "bilinearized system", admits a unique solution $(\nu, \psi, \rho) \in \mathcal{Y}$ :

$$
\begin{array}{lc}
\alpha \partial_{t} \nu+\partial_{t} \psi-\Delta \nu=g_{1}+g_{2} & \text { in } Q, \\
\beta \partial_{t} \psi-\Delta \psi-\nu=\chi \rho-F^{\prime \prime}(\bar{\varphi}) \psi-F^{(3)}(\bar{\varphi}) \xi^{\mathbf{h}} \xi^{\mathbf{k}} & \text { in } Q, \\
\partial_{t} \rho-\Delta \rho+\chi \Delta \psi=-g_{1} & \text { in } Q, \\
\partial_{\mathbf{n}} \nu=\partial_{\mathbf{n}} \psi=\partial_{\mathbf{n}} \rho=0 & \text { on } \Sigma, \\
\nu(0)=\psi(0)=\rho(0)=0 & \text { in } \Omega,
\end{array}
$$

where $g_{1}$ and $g_{2}$ are defined as

$$
\begin{aligned}
g_{1}= & P(\bar{\varphi})(\rho-\chi \psi-\nu)+P^{\prime}(\bar{\varphi}) \xi^{\mathbf{k}}\left(\theta^{\mathbf{h}}-\chi \xi^{\mathbf{h}}-\eta^{\mathbf{h}}\right) \\
& +P^{\prime \prime}(\bar{\varphi}) \xi^{\mathbf{k}} \xi^{\mathbf{h}}(\bar{\sigma}+\chi(1-\bar{\varphi})-\bar{\mu})+P^{\prime}(\bar{\varphi}) \psi(\bar{\sigma}+\chi(1-\bar{\varphi})-\bar{\mu}) \\
& +P^{\prime}(\bar{\varphi}) \xi^{\mathbf{h}}\left(\theta^{\mathbf{k}}-\chi \xi^{\mathbf{k}}-\eta^{\mathbf{k}}\right), \\
g_{2}= & -\mathbb{h}^{\prime \prime}(\bar{\varphi}) \xi^{\mathbf{k}} \xi^{\mathbf{h}} \bar{u}_{1}-\mathbb{h}^{\prime}(\bar{\varphi}) \xi^{\mathbf{h}} k_{1}-\mathbb{h}^{\prime}(\bar{\varphi}) \psi \bar{u}_{1}-\mathbb{h}^{\prime}(\bar{\varphi}) \xi^{\mathbf{k}} h_{1} .
\end{aligned}
$$

Moreover, there is some constant $D>0$, which only depends on the data of the system and $R$, such that

$$
\|(\nu, \psi, \rho)\|_{\mathcal{V}} \leq D\|\mathbf{h}\|_{L^{2}(0, T ; H)^{2}}\|\mathbf{k}\|_{L^{2}(0, T ; H)^{2}} \quad \forall \mathbf{h}, \mathbf{k} \in \mathcal{U}
$$

Proof. Writing $(\mu, \varphi, \sigma)$ in place of $(\nu, \psi, \rho)$, we see that the system (4.78)-(4.84) is of the form (4.6)-(4.10) with the specifications $\lambda_{1}=\lambda_{3}=1, \lambda_{2}=\lambda_{4}=0$, and

$$
\begin{aligned}
f_{1}= & P^{\prime}(\bar{\varphi}) \xi^{\mathbf{k}}\left(\theta^{\mathbf{h}}-\chi \xi^{\mathbf{h}}-\eta^{\mathbf{h}}\right)+P^{\prime \prime}(\bar{\varphi}) \xi^{\mathbf{k}} \xi^{\mathbf{h}}(\bar{\sigma}+\chi(1-\bar{\varphi})-\bar{\mu}) \\
& +P^{\prime}(\bar{\varphi}) \xi^{\mathbf{h}}\left(\theta^{\mathbf{k}}-\chi \xi^{\mathbf{k}}-\eta^{\mathbf{k}}\right)-\mathbb{h}^{\prime \prime}(\bar{\varphi}) \xi^{\mathbf{k}} \xi^{\mathbf{h}} \bar{u}_{1}-\mathbb{h}^{\prime}(\bar{\varphi}) \xi^{\mathbf{h}} k_{1}-\mathbb{h}^{\prime}(\bar{\varphi}) \xi^{\mathbf{k}} h_{1}
\end{aligned}
$$




$$
\begin{aligned}
f_{2}= & -F^{(3)}(\bar{\varphi}) \xi^{\mathbf{h}} \xi^{\mathbf{k}}, \\
f_{3}= & -P^{\prime}(\bar{\varphi}) \xi^{\mathbf{k}}\left(\theta^{\mathbf{h}}-\chi \xi^{\mathbf{h}}-\eta^{\mathbf{h}}\right)-P^{\prime \prime}(\bar{\varphi}) \xi^{\mathbf{k}} \xi^{\mathbf{h}}(\bar{\sigma}+\chi(1-\bar{\varphi})-\bar{\mu}) \\
& -P^{\prime}(\bar{\varphi}) \xi^{\mathbf{h}}\left(\theta^{\mathbf{k}}-\chi \xi^{\mathbf{k}}-\eta^{\mathbf{k}}\right) .
\end{aligned}
$$

Since $\left(\eta^{\mathbf{h}}, \xi^{\mathbf{h}}, \theta^{\mathbf{h}}\right),\left(\eta^{\mathbf{k}}, \xi^{\mathbf{k}}, \theta^{\mathbf{k}}\right) \in \mathbf{y}, \quad$ it $\quad$ is easily seen that $\left(f_{1}, f_{2}, f_{3}\right) \in L^{\infty}(Q) \times\left(H^{1}(0, T ; H)\right.$ $\left.\cap L^{\infty}(Q)\right) \times L^{\infty}(Q)$, and so the existence result follows from Lemma 4.1.

It remains to show (4.85). To this end, we first add the term $\psi$ on both sides of (4.79) and then test (4.78) by $\nu,(4.79)$ by $\partial_{t} \psi$, and (4.80) by $\rho$. Adding the resulting equalities, and integrating over time and by parts, we infer that

$$
\begin{aligned}
& \frac{\alpha}{2}\|\nu(t)\|^{2}+\int_{Q_{t}}|\nabla \nu|^{2}+\beta \int_{Q_{t}}\left|\partial_{t} \psi\right|^{2}+\frac{1}{2}\|\psi(t)\|_{V}^{2}+\frac{1}{2}\|\rho(t)\|^{2}+\int_{Q_{t}}|\nabla \rho|^{2} \\
& =\int_{Q_{t}} g_{1}(\nu-\rho)+\int_{Q_{t}}\left(\chi \rho-F^{\prime \prime}(\bar{\varphi}) \psi-F^{(3)}(\bar{\varphi}) \xi^{\mathbf{h}} \xi^{\mathbf{k}}+\psi\right) \partial_{t} \psi \\
& \quad+\chi \int_{Q_{t}} \nabla \psi \cdot \nabla \rho+\int_{Q_{t}} g_{2} \nu=: I_{1}+I_{2}+I_{3}+I_{4},
\end{aligned}
$$

with obvious meaning. Now, using the global estimates (2.38) and (2.40), the continuity of the embedding $V \subset L^{4}(\Omega)$, as well as Hölder's inequality and the estimate (4.43) for the linearized variables, we can easily check that

$$
\begin{aligned}
I_{1} \leq & c \int_{Q_{t}}\left(|\nu|^{2}+|\psi|^{2}+|\rho|^{2}\right)+c \int_{0}^{t}\left\|\xi^{\mathbf{k}}\right\|_{4}^{2}\left(\left\|\eta^{\mathbf{h}}\right\|_{4}^{2}+\left\|\xi^{\mathbf{h}}\right\|_{4}^{2}+\left\|\theta^{\mathbf{h}}\right\|_{4}^{2}\right) \mathrm{d} s \\
& +c \int_{0}^{t}\left\|\xi^{\mathbf{k}}\right\|_{4}^{2}\left\|\xi^{\mathbf{h}}\right\|_{4}^{2} \mathrm{~d} s+c \int_{0}^{t}\left\|\xi^{\mathbf{h}}\right\|_{4}^{2}\left(\left\|\eta^{\mathbf{k}}\right\|_{4}^{2}+\left\|\xi^{\mathbf{k}}\right\|_{4}^{2}+\left\|\theta^{\mathbf{k}}\right\|_{4}^{2}\right) \mathrm{d} s \\
\leq & c \int_{Q_{t}}\left(|\nu|^{2}+|\psi|^{2}+|\rho|^{2}\right)+c\|\mathbf{h}\|_{L^{2}(0, T ; H)^{2}}^{2}\|\mathbf{k}\|_{L^{2}(0, T ; H)^{2}}^{2} .
\end{aligned}
$$

Arguing similarly, where we also invoke Young's inequality, we obtain that

$$
I_{2} \leq \frac{\beta}{2} \int_{Q_{t}}\left|\partial_{t} \psi\right|^{2}+c \int_{Q_{t}}\left(|\psi|^{2}+|\rho|^{2}\right)+c\|\mathbf{h}\|_{L^{2}(0, T ; H)^{2}}^{2}\|\mathbf{k}\|_{L^{2}(0, T ; H)^{2}}^{2}
$$

as well as

$$
I_{3} \leq \frac{1}{2} \int_{Q_{t}}|\nabla \rho|^{2}+\frac{\chi^{2}}{2} \int_{Q_{t}}|\nabla \psi|^{2} .
$$

Finally, using also that $\bar{u}_{1}$ is bounded, we see that

$$
\begin{aligned}
I_{4} \leq & c \int_{Q_{t}}\left(|\nu|^{2}+|\psi|^{2}\right)+c \int_{0}^{t}\left\|\xi^{\mathbf{k}}\right\|_{4}^{2}\left\|\xi^{\mathbf{h}}\right\|_{4}^{2} \mathrm{~d} s \\
& +c \int_{0}^{t}\|\nu\|_{4}\left(\left\|\xi^{\mathbf{h}}\right\|_{4}\left\|k_{1}\right\|+\left\|\xi^{\mathbf{k}}\right\|_{4}\left\|h_{1}\right\|\right) \mathrm{d} s \\
\leq & (c+2 \delta) \int_{Q_{t}}\left(|\nu|^{2}+|\psi|^{2}\right)+2 \delta \int_{Q_{t}}|\nabla \nu|^{2}+c_{\delta}\|\mathbf{h}\|_{L^{2}(0, T ; H)^{2}}^{2}\|\mathbf{k}\|_{L^{2}(0, T ; H)^{2}}^{2} .
\end{aligned}
$$


Combining (4.86)-(4.90), and choosing $\delta>0$ appropriately small, we thus obtain from Gronwall's lemma that

$$
\begin{aligned}
& \|\nu\|_{L^{\infty}(0, T ; H) \cap L^{2}(0, T ; V)}+\|\psi\|_{H^{1}(0, T ; H) \cap L^{\infty}(0, T ; V)}+\|\rho\|_{L^{\infty}(0, T ; H) \cap L^{2}(0, T ; V)} \\
& \quad \leq c\|\mathbf{h}\|_{L^{2}(0, T ; H)^{2}}\|\mathbf{k}\|_{L^{2}(0, T ; H)^{2}} .
\end{aligned}
$$

Having shown this, we may bring $\beta \partial_{t} \psi$ to the right-hand side of (4.79). Elliptic regularity theory and the estimates shown above then yield that also

$$
\|\psi\|_{L^{2}\left(0, T ; W_{0}\right)} \leq c\|\mathbf{h}\|_{L^{2}(0, T ; H)^{2}}\|\mathbf{k}\|_{L^{2}(0, T ; H)^{2}},
$$

which concludes the proof of the assertion.

We now provide the announced independent proof for the form of the second-order derivative.

Theorem 4.7. Assume that (W1), (S1)-(S4), (2.32)-(2.34) are fulfilled, and let $\overline{\mathbf{u}} \in \mathcal{U}_{R}$ be given. Then the second Fréchet derivative $D^{2} \mathcal{S}(\overline{\mathbf{u}}) \in \mathcal{L}(\mathcal{U}, \mathcal{L}(\mathcal{U}, \mathcal{y}))$ is given, for every $\mathbf{h}=\left(h_{1}, h_{2}\right), \mathbf{k}=\left(k_{1}, k_{2}\right) \in \mathcal{U}$, by the identity $D^{2} \mathcal{S}(\overline{\mathbf{u}})(\mathbf{k})(\mathbf{h})=(\nu, \psi, \rho)$, where $(\nu, \psi, \rho)$ is the unique solution to the bilinearized system (4.78)-(4.84) introduced in Theorem 4.6.

Proof. By virtue of Theorem $4.3, D^{2} \mathcal{S}(\overline{\mathbf{u}})$ exists as an element of $\mathcal{L}(\mathcal{U}, \mathcal{L}(\mathcal{U}, \mathcal{y}))$. Now, the embedding of $y$ in $\mathcal{V}$ is continuous. Therefore, $\mathcal{S}$ is also twice continuously Fréchet differentiable between $\mathcal{U}$ and $\mathcal{V}$, and the expressions for the derivatives coincide. It thus suffices to work in the space $\mathcal{V}$. To this end, we recall that $\mathcal{U}_{R}$ is open in $\mathcal{U}$. Hence, there is some $K>0$ such that $\overline{\mathbf{u}}+\mathbf{k} \in \mathcal{U}_{R}$ whenever $\|\mathbf{k}\|_{\mathcal{U}} \leq K$. In the following, we always tacitly assume that the occurring increments $\mathbf{k}$ satisfy this condition.

To prove the claim, we proceed in a direct way, by showing that there exist some $\varepsilon>0$ and $\widehat{C}>0$ such that

$$
\begin{aligned}
& \left\|D \mathcal{S}(\overline{\mathbf{u}}+\mathbf{k})-D \mathcal{S}(\overline{\mathbf{u}})-D^{2} \mathcal{S}(\overline{\mathbf{u}})(\mathbf{k})\right\|_{\mathcal{L}(u, \mathcal{V})} \\
& \quad=\sup _{\|\mathbf{h}\|_{u}=1}\left\|\left(D \mathcal{S}(\overline{\mathbf{u}}+\mathbf{k})-D \mathcal{S}(\overline{\mathbf{u}})-D^{2} \mathcal{S}(\overline{\mathbf{u}})(\mathbf{k})\right)(\mathbf{h})\right\|_{\mathcal{V}} \leq \widehat{C}\|\mathbf{k}\|_{L^{2}(0, T ; H)^{2}}^{1+\varepsilon}
\end{aligned}
$$

At this point, we introduce some additional notation: for $\operatorname{arbitrary} \mathbf{h}, \mathbf{k} \in \mathcal{U}$, we define the linearized variables

$$
\left(\eta^{\mathbf{h}}, \xi^{\mathbf{h}}, \theta^{\mathbf{h}}\right):=D \mathcal{S}(\overline{\mathbf{u}})(\mathbf{h}), \quad\left(\bar{\eta}^{\mathbf{h}}, \bar{\xi}^{\mathbf{h}}, \bar{\theta}^{\mathbf{h}}\right):=D \mathcal{S}(\overline{\mathbf{u}}+\mathbf{k})(\mathbf{h})
$$

Notice that (4.43) implies that

$$
\left\|\left(\eta^{\mathbf{h}}, \xi^{\mathbf{h}}, \theta^{\mathbf{h}}\right)\right\|_{z} \leq c\|\mathbf{h}\|_{L^{2}(0, T ; H)^{2}}, \quad\left\|\left(\bar{\eta}^{\mathbf{h}}, \bar{\xi}^{\mathbf{h}}, \bar{\theta}^{\mathbf{h}}\right)\right\|_{z} \leq c\|\mathbf{h}\|_{L^{2}(0, T ; H)^{2}},
$$

where, here and in the remainder of this proof, $c>0$ denote constants that may depend on the data, but not on the choice of $\mathbf{k} \in \mathcal{U}$ with $\overline{\mathbf{u}}+\mathbf{k} \in \mathcal{U}_{R}$.

Next, we fix some $\mathbf{h} \in \mathcal{U}$ with $\|\mathbf{h}\| u=1$ and introduce the auxiliary variables

$$
\zeta=\bar{\eta}^{\mathbf{h}}-\eta^{\mathbf{h}}-\nu, \quad \phi=\bar{\xi}^{\mathbf{h}}-\xi^{\mathbf{h}}-\psi, \quad \omega=\bar{\theta}^{\mathbf{h}}-\theta^{\mathbf{h}}-\rho,
$$

where $(\nu, \psi, \rho)$ stands for the unique solution to the bilinearized system as obtained from Theorem 4.6. With this notation, we realize that $(4.92)$ reduces to

$$
\|(\zeta, \phi, \omega)\|_{\mathcal{V}}^{2} \leq \widehat{C}\|\mathbf{k}\|_{L^{2}(0, T ; H)^{2}}^{2+\varepsilon}
$$


which is the estimate we are going to show for some $\varepsilon>0$. To this end, we first observe that these new variables solve the initial-boundary value problem

$$
\begin{array}{ll}
\alpha \partial_{t} \zeta+\partial_{t} \phi-\Delta \zeta=\Lambda_{1}+\Lambda_{2} & \text { in } Q, \\
\beta \partial_{t} \phi-\Delta \phi-\zeta=\chi \omega-F^{\prime \prime}(\bar{\varphi}) \phi+\Lambda_{3} & \text { in } Q, \\
\partial_{t} \omega-\Delta \omega+\chi \Delta \phi=-\Lambda_{1} & \text { in } Q, \\
\partial_{\mathbf{n}} \zeta=\partial_{\mathbf{n}} \phi=\partial_{\mathbf{n}} \omega=0 & \text { on } \Sigma, \\
\zeta(0)=\phi(0)=\omega(0)=0 & \text { in } \Omega,
\end{array}
$$

where

$$
\begin{aligned}
\Lambda_{1}= & P(\bar{\varphi})(\omega-\chi \phi-\zeta)+\left(P\left(\bar{\varphi}^{\mathbf{k}}\right)-P(\bar{\varphi})\right)\left(\left(\bar{\theta}^{\mathbf{h}}-\theta^{\mathbf{h}}\right)-\chi\left(\bar{\xi}^{\mathbf{h}}-\xi^{\mathbf{h}}\right)-\left(\bar{\eta}^{\mathbf{h}}-\eta^{\mathbf{h}}\right)\right) \\
& +\left[P\left(\bar{\varphi}^{\mathbf{k}}\right)-P(\bar{\varphi})-P^{\prime}(\bar{\varphi}) \xi^{\mathbf{k}}\right]\left(\theta^{\mathbf{h}}-\chi \xi^{\mathbf{h}}-\eta^{\mathbf{h}}\right) \\
& +\left[P^{\prime}\left(\bar{\varphi}^{\mathbf{k}}\right)-P^{\prime}(\bar{\varphi})-P^{\prime \prime}(\bar{\varphi}) \xi^{\mathbf{k}}\right](\bar{\sigma}+\chi(1-\bar{\varphi})-\bar{\mu}) \xi^{\mathbf{h}} \\
& +P^{\prime}(\bar{\varphi})(\omega-\chi \phi-\zeta) \xi^{\mathbf{h}}+P^{\prime}(\bar{\varphi})(\bar{\sigma}+\chi(1-\bar{\varphi})-\bar{\mu}) \phi \\
& +\left(P^{\prime}\left(\bar{\varphi}^{\mathbf{k}}\right)-P^{\prime}(\bar{\varphi})\right)\left(\left(\bar{\sigma}^{\mathbf{k}}-\bar{\sigma}\right)-\chi\left(\bar{\varphi}^{\mathbf{k}}-\bar{\varphi}\right)-\left(\bar{\mu}^{\mathbf{k}}-\bar{\mu}\right)\right) \xi^{\mathbf{h}} \\
& +\left(P^{\prime}\left(\bar{\varphi}^{\mathbf{k}}\right)-P^{\prime}(\bar{\varphi})\right)(\bar{\sigma}+\chi(1-\bar{\varphi})-\bar{\mu})\left(\bar{\xi}^{\mathbf{h}}-\xi^{\mathbf{h}}\right) \\
& +P^{\prime}(\bar{\varphi})\left(\left(\bar{\sigma}^{\mathbf{k}}-\bar{\sigma}\right)-\chi\left(\bar{\varphi}^{\mathbf{k}}-\bar{\varphi}\right)-\left(\bar{\mu}^{\mathbf{k}}-\bar{\mu}\right)\right)\left(\bar{\xi}^{\mathbf{h}}-\xi^{\mathbf{h}}\right) \\
& +\left(P^{\prime}\left(\bar{\varphi}^{\mathbf{k}}\right)-P^{\prime}(\bar{\varphi})\right)\left(\left(\bar{\sigma}^{\mathbf{k}}-\bar{\sigma}\right)-\chi\left(\bar{\varphi}^{\mathbf{k}}-\bar{\varphi}\right)-\left(\bar{\mu}^{\mathbf{k}}-\bar{\mu}\right)\right)\left(\bar{\xi}^{\mathbf{h}}-\xi^{\mathbf{h}}\right), \\
\Lambda_{2}= & -h_{1}\left[\mathbb{h}^{(}\left(\bar{\varphi}^{\mathbf{k}}\right)-\mathbb{h}(\bar{\varphi})-\mathbb{h}^{\prime}(\bar{\varphi}) \xi^{\mathbf{k}}\right]-\left[\mathbb{h}^{\prime}\left(\bar{\varphi}^{\mathbf{k}}\right)-\mathbb{h}^{\prime}(\bar{\varphi})-\mathbb{h}^{\prime \prime}(\bar{\varphi}) \xi^{\mathbf{k}}\right] \xi^{\mathbf{h}} \bar{u}_{1}-\mathbb{h}^{\prime}(\bar{\varphi}) \phi \bar{u}_{1} \\
& -\left(\mathbb{h}^{\prime}\left(\bar{\varphi}^{\mathbf{k}}\right)-\mathbb{h}^{\prime}(\bar{\varphi})\right)\left(\bar{\xi}^{\mathbf{h}}-\xi^{\mathbf{h}}\right) \bar{u}_{1}-\left(\mathbb{h}^{\prime}\left(\bar{\varphi}^{\mathbf{k}}\right)-\mathbb{h}^{\prime}(\bar{\varphi})\right) \xi^{\mathbf{h}} k_{1} \\
& -\mathbb{h}^{\prime}(\bar{\varphi})\left(\bar{\xi}^{\mathbf{h}}-\xi^{\mathbf{h}}\right) k_{1}-\left(\mathbb{h}^{\prime}\left(\bar{\varphi}^{\mathbf{k}}\right)-\mathbb{h}^{\prime}(\bar{\varphi})\right)\left(\bar{\xi}^{\mathbf{h}}-\xi^{\mathbf{h}}\right) k_{1}, \\
\Lambda_{3}= & -\left[F^{\prime \prime}\left(\bar{\varphi}^{\mathbf{k}}\right)-F^{\prime \prime}(\bar{\varphi})-F^{(3)}(\bar{\varphi}) \xi^{\mathbf{k}}\right] \xi^{\mathbf{h}}-\left(F^{\prime \prime}\left(\bar{\varphi}^{\mathbf{k}}\right)-F^{\prime \prime}(\bar{\varphi})\right)\left(\bar{\xi}^{\mathbf{h}}-\xi^{\mathbf{h}}\right),
\end{aligned}
$$

and $(\bar{\mu}, \bar{\varphi}, \bar{\sigma})=\mathcal{S}(\overline{\mathbf{u}}),\left(\bar{\mu}^{\mathbf{k}}, \bar{\varphi}^{\mathbf{k}}, \bar{\sigma}^{\mathbf{k}}\right)=\mathcal{S}(\overline{\mathbf{u}}+\mathbf{k})$.

Before estimating in detail, let us recall that, owing to the continuous dependence results obtained in Theorem 2.3 and in Theorem 4.4 , and recalling that $\|\mathbf{h}\|_{\mathfrak{u}}=1$, we have that

$$
\begin{aligned}
& \left\|\left(\bar{\mu}^{\mathbf{k}}, \bar{\varphi}^{\mathbf{k}}, \bar{\sigma}^{\mathbf{k}}\right)-(\bar{\mu}, \bar{\varphi}, \bar{\sigma})\right\|_{\mathcal{Z}}+\left\|\left(\bar{\eta}^{\mathbf{h}}, \bar{\xi}^{\mathbf{h}}, \bar{\theta}^{\mathbf{h}}\right)-\left(\eta^{\mathbf{h}}, \xi^{\mathbf{h}}, \theta^{\mathbf{h}}\right)\right\|_{\mathcal{V}} \\
& \quad \leq c\left(1+\|\mathbf{h}\|_{L^{2}(0, T ; H)^{2}}\right)\|\mathbf{k}\|_{L^{2}(0, T ; H)^{2}} \leq c\|\mathbf{k}\|_{L^{2}(0, T ; H)^{2}}
\end{aligned}
$$

In particular, we point out that

$$
\left\|\bar{\varphi}^{\mathbf{k}}-\bar{\varphi}\right\|_{L^{\infty}(0, T ; V)}+\left\|\bar{\xi}^{\mathbf{h}}-\xi^{\mathbf{h}}\right\|_{L^{\infty}(0, T ; V)} \leq c\|\mathbf{k}\|_{L^{2}(0, T ; H)^{2}}
$$

We will use this estimate in the following at several places without further reference.

Moreover, let us recall that $(\bar{\mu}, \bar{\varphi}, \bar{\sigma})$ and $\left(\eta^{\mathbf{h}}, \xi^{\mathbf{h}}, \theta^{\mathbf{h}}\right)$, as solutions to (1.2)-(1.6) and (4.6)-(4.10) with $\lambda_{1}=$ $\lambda_{2}=1, \lambda_{3}=\lambda_{4}=0$, enjoy the bounds provided by (2.38)-(2.40) and

$$
\left\|\left(\eta^{\mathbf{h}}, \xi^{\mathbf{h}}, \theta^{\mathbf{h}}\right)\right\|_{y} \leq c,
$$


respectively. Notice also that the estimate (4.74) is valid for the arguments $\varphi_{1}=\bar{\varphi}^{\mathbf{k}}, \varphi_{2}=\bar{\varphi}$. In addition, we also owe to Taylor's formula with integral remainder for $P^{(i)}$ and $\mathbb{h}^{(i)}$ for $i=0,1$, which yields that

$$
\begin{aligned}
& P^{(i)}\left(\bar{\varphi}^{\mathbf{k}}\right)-P^{(i)}(\bar{\varphi})-P^{(i+1)}(\bar{\varphi}) \xi^{\mathbf{k}}=P^{(i+1)}(\bar{\varphi})\left(\bar{\varphi}^{\mathbf{k}}-\bar{\varphi}-\xi^{\mathbf{k}}\right)+Q_{i+2}^{\mathbf{k}}\left(\bar{\varphi}^{\mathbf{k}}-\bar{\varphi}\right)^{2}, \\
& \mathfrak{h}^{(i)}\left(\bar{\varphi}^{\mathbf{k}}\right)-\mathbb{h}^{(i)}(\bar{\varphi})-\mathbb{h}^{(i+1)}(\bar{\varphi}) \xi^{\mathbf{k}}=\mathbb{h}^{(i+1)}(\bar{\varphi})\left(\bar{\varphi}^{\mathbf{k}}-\bar{\varphi}-\xi^{\mathbf{k}}\right)+R_{i+2}^{\mathbf{k}}\left(\bar{\varphi}^{\mathbf{k}}-\bar{\varphi}\right)^{2},
\end{aligned}
$$

for $i=0,1$, where the remainders $Q_{i}^{\mathbf{k}}, R_{i}^{\mathbf{k}}$ are uniformly bounded and defined by

$$
Q_{i+2}^{\mathbf{k}}=\int_{0}^{1} P^{(i+2)}\left(\bar{\varphi}+s\left(\bar{\varphi}^{\mathbf{k}}-\bar{\varphi}\right)\right)(1-s) \mathrm{d} s, \quad R_{i+2}^{\mathbf{k}}=\int_{0}^{1} \mathbb{h}^{(i+2)}\left(\bar{\varphi}+s\left(\bar{\varphi}^{\mathbf{k}}-\bar{\varphi}\right)\right)(1-s) \mathrm{d} s
$$

Namely, there exists a positive constant $R^{*}$ such that, for all $\mathbf{k} \in \mathcal{U}$ with $\overline{\mathbf{u}}+\mathbf{k} \in \mathfrak{U}_{R}$,

$$
\left\|Q_{2}^{\mathbf{k}}\right\|_{L^{\infty}(Q)}+\left\|Q_{3}^{\mathbf{k}}\right\|_{L^{\infty}(Q)}+\left\|R_{2}^{\mathbf{k}}\right\|_{L^{\infty}(Q)}+\left\|R_{3}^{\mathbf{k}}\right\|_{L^{\infty}(Q)} \leq R^{*} .
$$

Moreover, owing to Remark 4.5, we also have that

$$
\left\|\left(\bar{\mu}^{\mathbf{k}}-\bar{\mu}-\eta^{\mathbf{k}}, \bar{\varphi}^{\mathbf{k}}-\bar{\varphi}-\xi^{\mathbf{k}}, \bar{\sigma}^{\mathbf{k}}-\bar{\sigma}-\theta^{\mathbf{k}}\right)\right\|_{z} \leq c\|\mathbf{k}\|_{L^{2}(0, T ; H)^{2}}^{2} .
$$

Thus, we test (4.95) by $\zeta,(4.96)$, to which we add on both sides the term $\phi$, by $\partial_{t} \phi$, and (4.97) by $\omega$, add the resulting equations, and integrate over time and by parts to obtain that

$$
\begin{aligned}
& \frac{\alpha}{2}\|\zeta(t)\|^{2}+\int_{Q_{t}}|\nabla \zeta|^{2}+\beta \int_{Q_{t}}\left|\partial_{t} \phi\right|^{2}+\frac{1}{2}\|\phi(t)\|_{V}^{2}+\frac{1}{2}\|\omega(t)\|^{2}+\int_{Q_{t}}|\nabla \omega|^{2} \\
& =\int_{Q_{t}} \Lambda_{1}(\zeta-\omega)+\int_{Q_{t}} \Lambda_{2} \zeta+\int_{Q_{t}}\left(\chi \omega-F^{\prime \prime}(\bar{\varphi}) \phi+\Lambda_{3}+\phi\right) \partial_{t} \phi \\
& \quad+\chi \int_{Q_{t}} \nabla \phi \cdot \nabla \omega=: I_{1}+I_{2}+I_{3}+I_{4} .
\end{aligned}
$$

Using the Young and Hölder inequalities, the above properties, the continuous embedding $V \subset L^{6}(\Omega),(4.65)$, the general property (4.74) with $p=4$ and $p=6$, and (4.100), as well as Taylor's formulae (4.101)-(4.102), we infer that

$$
\begin{aligned}
I_{1} \leq & c \int_{Q_{t}}\left(|\omega|^{2}+|\phi|^{2}+|\zeta|^{2}\right)+c \int_{0}^{t}\left\|\bar{\varphi}^{\mathbf{k}}-\bar{\varphi}\right\|_{V}^{2}\left(\left\|\bar{\theta}^{\mathbf{h}}-\theta^{\mathbf{h}}\right\|_{4}^{2}+\left\|\bar{\xi}^{\mathbf{h}}-\xi^{\mathbf{h}}\right\|_{4}^{2}+\left\|\bar{\eta}^{\mathbf{h}}-\eta^{\mathbf{h}}\right\|_{4}^{2}\right) \mathrm{d} s \\
& +c\left(\left\|\theta^{\mathbf{h}}\right\|_{L^{\infty}(Q)}^{2}+\left\|\xi^{\mathbf{h}}\right\|_{L^{\infty}(Q)}^{2}+\left\|\eta^{\mathbf{h}}\right\|_{L^{\infty}(Q)}^{2}\right) \int_{0}^{t}\left(\|\mathbf{k}\|_{L^{2}(0, s ; H)^{2}}^{4}+\left\|\bar{\varphi}^{\mathbf{k}}-\bar{\varphi}\right\|_{4}^{4}\right) \mathrm{d} s \\
& +c\left(\|\bar{\sigma}\|_{L^{\infty}(Q)}^{2}+\|\bar{\varphi}\|_{L^{\infty}(Q)}^{2}+\|\bar{\mu}\|_{L^{\infty}(Q)}^{2}+1\right)\left\|\xi^{\mathbf{h}}\right\|_{L^{\infty}(Q)}^{2} \int_{0}^{t}\left(\|\mathbf{k}\|_{L^{2}(0, s ; H)^{2}}^{4}+\left\|\bar{\varphi}^{\mathbf{k}}-\bar{\varphi}\right\|_{4}^{4}\right) \mathrm{d} s \\
& +c\left(\left\|\xi^{\mathbf{h}}\right\|_{L^{\infty}(Q)}^{2}+1\right) \int_{Q_{t}}\left(|\omega|^{2}+|\phi|^{2}+|\zeta|^{2}\right) \\
& +c\left\|\xi^{\mathbf{h}}\right\|_{L^{\infty}(Q)}^{2} \int_{0}^{t}\left\|\bar{\varphi}^{\mathbf{k}}-\bar{\varphi}\right\|_{V}^{2}\left(\left\|\bar{\sigma}^{\mathbf{k}}-\bar{\sigma}\right\|_{4}^{2}+\left\|\bar{\varphi}^{\mathbf{k}}-\bar{\varphi}\right\|_{4}^{2}+\left\|\bar{\mu}^{\mathbf{k}}-\bar{\mu}\right\|_{4}^{2}\right) \mathrm{d} s \\
& +c\left(\|\bar{\sigma}\|_{L^{\infty}(Q)}^{2}+\|\bar{\varphi}\|_{L^{\infty}(Q)}^{2}+\|\bar{\mu}\|_{L^{\infty}(Q)}^{2}+1\right) \int_{0}^{t}\left\|\bar{\varphi}^{\mathbf{k}}-\bar{\varphi}\right\|_{V}^{2}\left\|\bar{\xi}^{\mathbf{h}}-\xi^{\mathbf{h}}\right\|_{4}^{2} \mathrm{~d} s
\end{aligned}
$$




$$
\begin{aligned}
& +c \int_{0}^{t}\left(\left\|\bar{\sigma}^{\mathbf{k}}-\bar{\sigma}\right\|_{4}^{2}+\left\|\bar{\varphi}^{\mathbf{k}}-\bar{\varphi}\right\|_{4}^{2}+\left\|\bar{\mu}^{\mathbf{k}}-\bar{\mu}\right\|_{4}^{2}\right)\left\|\bar{\xi}^{\mathbf{h}}-\xi^{\mathbf{h}}\right\|_{4}^{2} \mathrm{~d} s \\
& +c \int_{0}^{t}\left\|\bar{\varphi}^{\mathbf{k}}-\bar{\varphi}\right\|_{V}^{2}\left(\left\|\bar{\sigma}^{\mathbf{k}}-\bar{\sigma}\right\|_{6}^{2}+\left\|\bar{\varphi}^{\mathbf{k}}-\bar{\varphi}\right\|_{6}^{2}+\left\|\bar{\mu}^{\mathbf{k}}-\bar{\mu}\right\|_{6}^{2}\right)\left\|\bar{\xi}^{\mathbf{h}}-\xi^{\mathbf{h}}\right\|_{6}^{2} \mathrm{~d} s \\
\leq & c \int_{0}^{t}\left(\|\omega\|^{2}+\|\phi\|^{2}+\|\zeta\|^{2}\right) \mathrm{d} s+c\|\mathbf{k}\|_{L^{2}(0, T ; H)^{2}}^{4} .
\end{aligned}
$$

Similar computations show that also

$$
\begin{aligned}
I_{2} \leq & c \int_{Q_{t}}|\zeta|^{2}+c\left\|h_{1}\right\|_{L^{\infty}(Q)}^{2} \int_{0}^{t}\left(\|\mathbf{k}\|_{L^{2}(0, s ; H)^{2}}^{4}+\left\|\bar{\varphi}^{\mathbf{k}}-\bar{\varphi}\right\|_{4}^{4}\right) \mathrm{d} s \\
& +c\left\|\xi^{\mathbf{h}}\right\|_{L^{\infty}(Q)}^{2}\left\|\bar{u}_{1}\right\|_{L^{\infty}(Q)}^{2} \int_{0}^{t}\left(\|\mathbf{k}\|_{L^{2}(0, s ; H)^{2}}^{4}+\left\|\bar{\varphi}^{\mathbf{k}}-\bar{\varphi}\right\|_{4}^{4}\right) \mathrm{d} s \\
& +c\left\|\bar{u}_{1}\right\|_{L^{\infty}(Q)}^{2} \int_{Q_{t}}|\phi|^{2}+c\left\|\bar{u}_{1}\right\|_{L^{\infty}(Q)}^{2} \int_{0}^{t}\left\|\bar{\varphi}^{\mathbf{k}}-\bar{\varphi}\right\|_{4}^{2}\left\|\bar{\xi}^{\mathbf{h}}-\xi^{\mathbf{h}}\right\|_{4}^{2} \mathrm{~d} s \\
& +\delta \int_{0}^{t}\|\zeta\|_{V}^{2} \mathrm{~d} s+c_{\delta}\left\|\xi^{\mathbf{h}}\right\|_{L^{\infty}(Q)}^{2} \int_{0}^{t}\left\|\bar{\varphi}^{\mathbf{k}}-\bar{\varphi}\right\|_{4}^{2}\left\|k_{1}\right\|^{2} \mathrm{~d} s \\
& +\delta \int_{0}^{t}\|\zeta\|_{V}^{2} \mathrm{~d} s+c_{\delta} \int_{0}^{t}\left\|\bar{\xi}^{\mathbf{h}}-\xi^{\mathbf{h}}\right\|_{4}^{2}\left\|k_{1}\right\|^{2} \mathrm{~d} s \\
& +\delta \int_{0}^{t}\|\zeta\|_{V}^{2} \mathrm{~d} s+c_{\delta} \int_{0}^{t}\left\|\bar{\varphi}^{\mathbf{k}}-\bar{\varphi}\right\|_{6}^{2}\left\|\bar{\xi}^{\mathbf{h}}-\xi^{\mathbf{h}}\right\|_{6}^{2}\left\|k_{1}\right\|^{2} \mathrm{~d} s \\
\leq & 3 \delta \int_{Q_{t}}|\nabla \zeta|^{2}+c_{\delta} \int_{Q_{t}}|\zeta|^{2}+c \int_{Q_{t}}|\phi|^{2}+c\|\mathbf{k}\|_{L^{2}(0, T ; H)^{2},}^{4}
\end{aligned}
$$

for a positive $\delta$ yet to be determined. As for $I_{3}$, we use (2.40) and, for the first term in $\Lambda_{3}$, we can argue as in (4.101)-(4.102) and the following lines. Then, we have that

$$
\begin{aligned}
I_{3} \leq & \delta \int_{Q_{t}}\left|\partial_{t} \phi\right|^{2}+c_{\delta} \int_{Q_{t}}\left(|\omega|^{2}+|\phi|^{2}\right)+c_{\delta} \int_{Q_{t}}\left|\Lambda_{3}\right|^{2} \\
\leq & \delta \int_{Q_{t}}\left|\partial_{t} \phi\right|^{2}+c_{\delta} \int_{Q_{t}}\left(|\omega|^{2}+|\phi|^{2}\right)+c\left\|\xi^{\mathbf{h}}\right\|_{L^{\infty}(Q)}^{2} \int_{0}^{t}\left(\|\mathbf{k}\|_{L^{2}(0, s ; H)^{2}}^{4}\left\|\bar{\varphi}^{\mathbf{k}}-\bar{\varphi}\right\|_{4}^{4}\right) \mathrm{d} s \\
& +c_{\delta} \int_{0}^{t}\left\|\bar{\varphi}^{\mathbf{k}}-\bar{\varphi}\right\|_{V}^{2}\left\|\bar{\xi}^{\mathbf{h}}-\xi^{\mathbf{h}}\right\|_{4}^{2} \mathrm{~d} s \\
\leq & \delta \int_{Q_{t}}\left|\partial_{t} \phi\right|^{2}+c_{\delta} \int_{Q_{t}}\left(|\omega|^{2}+|\phi|^{2}\right)+c_{\delta}\|\mathbf{k}\|_{L^{2}(0, T ; H)^{2}}^{4},
\end{aligned}
$$

and, as for $I_{4}$, we see that

$$
I_{4} \leq \frac{1}{2} \int_{Q_{t}}|\nabla \omega|^{2}+\frac{\chi^{2}}{2} \int_{Q_{t}}|\nabla \phi|^{2}
$$

where the first term can be absorbed on the left-hand side of (4.103). Therefore, collecting the above estimates and choosing $\delta>0$ small enough, we obtain from Gronwall's lemma that

$$
\begin{aligned}
& \|\zeta\|_{L^{\infty}(0, T ; H) \cap L^{2}(0, T ; V)}+\|\phi\|_{H^{1}(0, T ; H) \cap L^{\infty}(0, T ; V)}+\|\omega\|_{L^{\infty}(0, T ; H) \cap L^{2}(0, T ; V)} \\
& \quad \leq c\|\mathbf{k}\|_{L^{2}(0, T ; H)^{2}}^{2} .
\end{aligned}
$$


With this estimate shown, we can move the term $\beta \partial_{t} \phi$ to the right-hand side of (4.96), and it readily follows from elliptic regularity theory that also

$$
\|\phi\|_{L^{2}\left(0, T ; W_{0}\right)} \leq c\|\mathbf{k}\|_{L^{2}(0, T ; H)^{2}}^{2} .
$$

In conclusion, the estimate (4.94) is valid with $\varepsilon=2$. This finishes the proof of the assertion.

The last result we present in this section will be useful later on to handle the second-order sufficient optimality conditions and establishes the Lipschitz continuity of $D^{2} \mathcal{S}$ in a suitable sense.

Theorem 4.8. Suppose that the conditions (W1), (S1)-(S4), (C3) and (2.32) are fulfilled. Moreover, let the initial data $\left(\mu_{0}, \varphi_{0}, \sigma_{0}\right)$ satisfy $(2.33)-(2.34)$, and let $\overline{\mathbf{u}}^{i}=\left(\bar{u}_{1}^{i}, \bar{u}_{2}^{i}\right) \in \mathcal{U}_{R}$ be controls with the associated states $\left(\bar{\mu}_{i}, \bar{\varphi}_{i}, \bar{\sigma}_{i}\right)=\mathcal{S}\left(\overline{\mathbf{u}}^{i}\right), i=1,2$. Furthermore, let $\mathbf{h}, \mathbf{k} \in \mathcal{U}$ be admissible increments with the corresponding linearized variables $\left(\eta_{i}^{\mathbf{h}}, \xi_{i}^{\mathbf{h}}, \theta_{i}^{\mathbf{h}}\right)=D \mathcal{S}\left(\overline{\mathbf{u}}_{i}\right)(\mathbf{h}),\left(\eta_{i}^{\mathbf{k}}, \xi_{i}^{\mathbf{k}}, \theta_{i}^{\mathbf{k}}\right)=D \mathcal{S}\left(\overline{\mathbf{u}}_{i}\right)(\mathbf{k})$, and bilinearized variables $\left(\nu_{i}, \psi_{i}, \rho_{i}\right)=$ $D^{2} \mathcal{S}\left(\overline{\mathbf{u}}_{i}\right)(\mathbf{h})(\mathbf{k}), i=1,2$. Then it holds that

$$
\begin{aligned}
& \left\|\left(D^{2} \mathcal{S}\left(\overline{\mathbf{u}}^{1}\right)-D^{2} \mathcal{S}\left(\overline{\mathbf{u}}^{2}\right)\right)(\mathbf{h})(\mathbf{k})\right\|_{\mathcal{V}} \\
& \quad \leq K\left\|\overline{\mathbf{u}}^{1}-\overline{\mathbf{u}}^{2}\right\|_{L^{2}(0, T ; H)^{2}}\|\mathbf{h}\|_{L^{2}(0, T ; H)^{2}}\|\mathbf{k}\|_{L^{2}(0, T ; H)^{2}}
\end{aligned}
$$

with a constant $K>0$ that does not depend on the choice of $\mathbf{h}, \mathbf{k} \in \mathfrak{U}_{R}$.

Proof. By virtue of Theorem 4.7, (4.104) directly follows once we show the existence of some $c>0$ such that

$$
\left\|\left(\nu_{1}, \psi_{1}, \rho_{1}\right)-\left(\nu_{2}, \psi_{2}, \rho_{2}\right)\right\| \nu \leq c\left\|\overline{\mathbf{u}}^{1}-\overline{\mathbf{u}}^{2}\right\|_{L^{2}(0, T ; H)^{2}}\|\mathbf{h}\|_{L^{2}(0, T ; H)^{2}}\|\mathbf{k}\|_{L^{2}(0, T ; H)^{2}},
$$

which is the estimate we are going to check. To this end, we set

$$
\begin{array}{ll}
\bar{\mu}=\bar{\mu}_{1}-\bar{\mu}_{2}, \quad \bar{\varphi}=\bar{\varphi}_{1}-\bar{\varphi}_{2}, \quad \bar{\sigma}=\bar{\sigma}_{1}-\bar{\sigma}_{2}, \\
\nu=\nu_{1}-\nu_{2}, \quad \psi=\psi_{1}-\psi_{2}, \quad \rho=\rho_{1}-\rho_{2},
\end{array}
$$

and observe that the triple of differences $(\nu, \psi, \rho)$ solves the system

$$
\begin{array}{ll}
\alpha \partial_{t} \nu+\partial_{t} \psi-\Delta \nu=f_{1}+f_{2} & \text { in } Q, \\
\beta \partial_{t} \psi-\Delta \psi-\nu=\chi_{\rho}+f_{3} & \text { in } Q, \\
\partial_{t} \rho-\Delta \rho+\chi \Delta \psi=-f_{1} & \text { in } Q, \\
\partial_{\mathbf{n}} \nu=\partial_{\mathbf{n}} \psi=\partial_{\mathbf{n}} \rho=0 & \text { on } \Sigma, \\
\nu(0)=\psi(0)=\rho(0)=0 & \text { in } \Omega,
\end{array}
$$

with

$$
\begin{aligned}
f_{1}= & \left(P\left(\bar{\varphi}_{1}\right)-P\left(\bar{\varphi}_{2}\right)\right)\left(\rho_{1}-\chi \psi_{1}-\nu_{1}\right)+P\left(\bar{\varphi}_{2}\right)(\rho-\chi \psi-\nu) \\
& +\left(P^{\prime}\left(\bar{\varphi}_{1}\right)-P^{\prime}\left(\bar{\varphi}_{2}\right)\right) \xi_{1}^{\mathbf{k}}\left(\theta_{1}^{\mathbf{h}}-\chi \xi_{1}^{\mathbf{h}}-\eta_{1}^{\mathbf{h}}\right)+P^{\prime}\left(\bar{\varphi}_{2}\right)\left(\xi_{1}^{\mathbf{k}}-\xi_{2}^{\mathbf{k}}\right)\left(\theta_{1}^{\mathbf{h}}-\chi \xi_{1}^{\mathbf{h}}-\eta_{1}^{\mathbf{h}}\right) \\
& +P^{\prime}\left(\bar{\varphi}_{2}\right) \xi_{2}^{\mathbf{k}}\left(\left(\theta_{1}^{\mathbf{h}}-\theta_{2}^{\mathbf{h}}\right)-\chi\left(\xi_{1}^{\mathbf{h}}-\xi_{2}^{\mathbf{h}}\right)-\left(\eta_{1}^{\mathbf{h}}-\eta_{2}^{\mathbf{h}}\right)\right) \\
& +\left(P^{\prime \prime}\left(\bar{\varphi}_{1}\right)-P^{\prime \prime}\left(\bar{\varphi}_{2}\right)\right) \xi_{1}^{\mathbf{k}} \xi_{1}^{\mathbf{h}}\left(\bar{\sigma}_{1}+\chi\left(1-\bar{\varphi}_{1}\right)-\bar{\mu}_{1}\right) \\
& +P^{\prime \prime}\left(\bar{\varphi}_{2}\right)\left(\xi_{1}^{\mathbf{k}}-\xi_{2}^{\mathbf{k}}\right) \xi_{1}^{\mathbf{h}}\left(\bar{\sigma}_{1}+\chi\left(1-\bar{\varphi}_{1}\right)-\bar{\mu}_{1}\right) \\
& +P^{\prime \prime}\left(\bar{\varphi}_{2}\right) \xi_{2}^{\mathbf{k}}\left(\xi_{1}^{\mathbf{h}}-\xi_{2}^{\mathbf{h}}\right)\left(\bar{\sigma}_{1}+\chi\left(1-\bar{\varphi}_{1}\right)-\bar{\mu}_{1}\right) \\
& +P^{\prime \prime}\left(\bar{\varphi}_{2}\right) \xi_{2}^{\mathbf{k}} \xi_{2}^{\mathbf{h}}(\bar{\sigma}-\chi \bar{\varphi}-\bar{\mu})+\left(P^{\prime}\left(\bar{\varphi}_{1}\right)-P^{\prime}\left(\bar{\varphi}_{2}\right)\right) \psi_{1}\left(\bar{\sigma}_{1}+\chi\left(1-\bar{\varphi}_{1}\right)-\bar{\mu}_{1}\right)
\end{aligned}
$$




$$
\begin{aligned}
& +P^{\prime}\left(\bar{\varphi}_{2}\right) \psi\left(\bar{\sigma}_{1}+\chi\left(1-\bar{\varphi}_{1}\right)-\bar{\mu}_{1}\right)+P^{\prime}\left(\bar{\varphi}_{2}\right) \psi_{2}(\bar{\sigma}-\chi \bar{\varphi}-\bar{\mu}) \\
& +\left(P^{\prime}\left(\bar{\varphi}_{1}\right)-P^{\prime}\left(\bar{\varphi}_{2}\right)\right) \xi_{1}^{\mathbf{h}}\left(\theta_{1}^{\mathbf{k}}-\chi \xi_{1}^{\mathbf{k}}-\eta_{1}^{\mathbf{k}}\right)+P^{\prime}\left(\bar{\varphi}_{2}\right)\left(\xi_{1}^{\mathbf{h}}-\xi_{2}^{\mathbf{h}}\right)\left(\theta_{1}^{\mathbf{k}}-\chi \xi_{1}^{\mathbf{k}}-\eta_{1}^{\mathbf{k}}\right) \\
& +P^{\prime}\left(\bar{\varphi}_{2}\right) \xi_{2}^{\mathbf{h}}\left(\left(\theta_{1}^{\mathbf{k}}-\theta_{2}^{\mathbf{k}}\right)-\chi\left(\xi_{1}^{\mathbf{k}}-\xi_{2}^{\mathbf{k}}\right)-\left(\eta_{1}^{\mathbf{k}}-\eta_{2}^{\mathbf{k}}\right)\right), \\
f_{2}= & -\left(\mathbb{h}^{\prime \prime}\left(\bar{\varphi}_{1}\right)-\mathbb{h}^{\prime \prime}\left(\bar{\varphi}_{2}\right)\right) \xi_{1}^{\mathbf{k}} \xi_{1}^{\mathbf{h}} \bar{u}_{1}^{1}-\mathbb{h}^{\prime \prime}\left(\bar{\varphi}_{2}\right)\left(\xi_{1}^{\mathbf{k}}-\xi_{2}^{\mathbf{k}}\right) \xi_{1}^{\mathbf{h}} \bar{u}_{1}^{1}-\mathbb{h}^{\prime \prime}\left(\bar{\varphi}_{2}\right) \xi_{2}^{\mathbf{k}}\left(\xi_{1}^{\mathbf{h}}-\xi_{2}^{\mathbf{h}}\right) \bar{u}_{1}^{1} \\
& -\mathbb{h}^{\prime \prime}\left(\bar{\varphi}_{2}\right) \xi_{2}^{\mathbf{k}} \xi_{2}^{\mathbf{h}}\left(\bar{u}_{1}^{1}-\bar{u}_{1}^{2}\right)-\left(\mathfrak{h}^{\prime}\left(\bar{\varphi}_{1}\right)-\mathbb{h}^{\prime}\left(\bar{\varphi}_{2}\right)\right) \xi_{1}^{\mathbf{h}} k_{1}-\mathbb{h}^{\prime}\left(\bar{\varphi}_{2}\right)\left(\xi_{1}^{\mathbf{h}}-\xi_{2}^{\mathbf{h}}\right) k_{1} \\
& -\left(\mathbb{h}^{\prime}\left(\bar{\varphi}_{1}\right)-\mathbb{h}^{\prime}\left(\bar{\varphi}_{2}\right)\right) \psi_{1} \bar{u}_{1}^{1}-\mathbb{h}^{\prime}\left(\bar{\varphi}_{2}\right) \psi \bar{u}_{1}^{1}-\mathbb{h}^{\prime}\left(\bar{\varphi}_{2}\right) \psi_{2}\left(\bar{u}_{1}^{1}-\bar{u}_{1}^{2}\right) \\
& -\left(\mathbb{h}^{\prime}\left(\bar{\varphi}_{1}\right)-\mathbb{h}^{\prime}\left(\bar{\varphi}_{2}\right)\right) \xi_{1}^{\mathbf{k}} h_{1}-\mathbb{h}^{\prime}\left(\bar{\varphi}_{2}\right)\left(\xi_{1}^{\mathbf{k}}-\xi_{2}^{\mathbf{k}}\right) h_{1}, \\
f_{3}= & -\left(F^{\prime \prime}\left(\bar{\varphi}_{1}\right)-F^{\prime \prime}\left(\bar{\varphi}_{2}\right)\right) \psi_{1}-F^{\prime \prime}\left(\bar{\varphi}_{2}\right) \psi-\left(F^{(3)}\left(\bar{\varphi}_{1}\right)-F^{(3)}\left(\bar{\varphi}_{2}\right)\right) \xi_{1}^{\mathbf{h}} \xi_{1}^{\mathbf{k}} \\
& -F^{(3)}\left(\bar{\varphi}_{2}\right)\left(\xi_{1}^{\mathbf{h}}-\xi_{2}^{\mathbf{h}}\right) \xi_{1}^{\mathbf{k}}-F^{(3)}\left(\bar{\varphi}_{2}\right) \xi_{2}^{\mathbf{h}}\left(\xi_{1}^{\mathbf{k}}-\xi_{2}^{\mathbf{k}}\right) .
\end{aligned}
$$

Moreover, due to (2.41) and (4.65), we have (4.73) as well as

$$
\begin{aligned}
& \left\|\eta_{1}^{\mathbf{h}}-\eta_{2}^{\mathbf{h}}\right\|_{L^{\infty}(0, T ; H) \cap L^{2}(0, T ; V)}+\left\|\xi_{1}^{\mathbf{h}}-\xi_{2}^{\mathbf{h}}\right\|_{H^{1}(0, T ; H) \cap L^{\infty}(0, T ; V) \cap L^{2}\left(0, T ; W_{0}\right)} \\
& +\left\|\theta_{1}^{\mathbf{h}}-\theta_{2}^{\mathbf{h}}\right\|_{L^{\infty}(0, T ; H) \cap L^{2}(0, T ; V)} \leq c\left\|\overline{\mathbf{u}}^{1}-\overline{\mathbf{u}}^{2}\right\|_{L^{2}(0, T ; H)^{2}}\|\mathbf{h}\|_{L^{2}(0, T ; H)^{2}}
\end{aligned}
$$

and the corresponding estimate for $\mathbf{k}$. Also, owing to Theorems 2.3, 4.3 and 4.6, for $i=1,2$ it is clear that $\left(\bar{\mu}_{i}, \bar{\varphi}_{i}, \bar{\sigma}_{i}\right),\left(\eta_{i}^{\mathbf{h}}, \xi_{i}^{\mathbf{h}}, \theta_{i}^{\mathbf{h}}\right),\left(\eta_{i}^{\mathbf{k}}, \xi_{i}^{\mathbf{k}}, \theta_{i}^{\mathbf{k}}\right)$, and also $\left(\nu_{i}, \psi_{i}, \rho_{i}\right)$ belong to $\boldsymbol{y}$. Moreover, for both $\left(\eta_{i}^{\mathbf{h}}, \xi_{i}^{\mathbf{h}}, \theta_{i}^{\mathbf{h}}\right)$ and $\left(\eta_{i}^{\mathbf{k}}, \xi_{i}^{\mathbf{k}}, \theta_{i}^{\mathbf{k}}\right), i=1,2$, we have (4.67) with the corresponding increment, and from Theorem 4.6, that

$$
\left\|\left(\nu_{i}, \psi_{i}, \rho_{i}\right)\right\|_{\mathcal{V}} \leq c\|\mathbf{h}\|_{L^{2}(0, T ; H)^{2}}\|\mathbf{k}\|_{L^{2}(0, T ; H)^{2}}, \quad i=1,2 .
$$

We are now ready to proceed by arguing in a similar fashion as in Theorem 4.4 in order to check (4.105).

First ESTimate: To begin with, we multiply (4.106) by $\nu,(4.107)$, to which we add to both sides the term $\psi$, by $\partial_{t} \psi$, and (4.108) by $\rho$. Then we add the resulting equalities and integrate $Q_{t}$ and by parts to obtain

$$
\begin{aligned}
& \frac{\alpha}{2}\|\nu(t)\|^{2}+\int_{Q_{t}}|\nabla \nu|^{2}+\beta \int_{Q_{t}}\left|\partial_{t} \psi\right|^{2}+\frac{1}{2}\|\psi(t)\|_{V}^{2}+\frac{1}{2}\|\rho(t)\|^{2}+\int_{Q_{t}}|\nabla \rho|^{2} \\
& =\int_{Q_{t}} f_{1}(\nu-\rho)+\int_{Q_{t}} f_{2} \nu+\int_{Q_{t}}\left(\chi \rho+\psi+f_{3}\right) \partial_{t} \psi \\
& \quad+\chi \int_{Q_{t}} \nabla \psi \cdot \nabla \rho=: I_{1}+I_{2}+I_{3}+I_{4} .
\end{aligned}
$$

Then, lengthy, but straightforward, calculations along the same lines as in the proofs of the previous theorems, eventually lead to the following estimates (with $\delta>0$ yet to be chosen):

$$
\begin{aligned}
I_{1} \leq & c \int_{Q_{t}}\left(|\nu|^{2}+|\psi|^{2}+|\rho|^{2}\right)+c\left\|\overline{\mathbf{u}}^{1}-\overline{\mathbf{u}}^{2}\right\|_{L^{2}(0, T ; H)^{2}}^{2}\|\mathbf{h}\|_{L^{2}(0, T ; H)^{2}}^{2}\|\mathbf{k}\|_{L^{2}(0, T ; H)^{2}}^{2}, \\
I_{2} \leq & \delta \int_{Q_{t}}|\nabla \nu|^{2}+c_{\delta} \int_{Q_{t}}|\nu|^{2}+c \int_{Q_{t}}|\psi|^{2} \\
& +c_{\delta}\left\|\overline{\mathbf{u}}^{1}-\overline{\mathbf{u}}^{2}\right\|_{L^{2}(0, T ; H)^{2}}^{2}\|\mathbf{h}\|_{L^{2}(0, T ; H)^{2}}^{2}\|\mathbf{k}\|_{L^{2}(0, T ; H)^{2}}^{2}, \\
I_{3} \leq & \delta \int_{Q_{t}}\left|\partial_{t} \psi\right|^{2}+c_{\delta} \int_{Q_{t}}\left(|\rho|^{2}+|\psi|^{2}\right)+c_{\delta}\left\|\overline{\mathbf{u}}^{1}-\overline{\mathbf{u}}^{2}\right\|_{L^{2}(0, T ; H)^{2}}^{2}\|\mathbf{h}\|_{L^{2}(0, T ; H)^{2}}^{2}\|\mathbf{k}\|_{L^{2}(0, T ; H)^{2}}^{2},
\end{aligned}
$$




$$
I_{4} \leq \delta \int_{Q_{t}}|\nabla \rho|^{2}+c_{\delta} \int_{Q_{t}}|\nabla \psi|^{2} .
$$

Therefore, choosing $\delta>0$ small enough, and invoking Gronwall's lemma, we conclude that

$$
\begin{aligned}
& \|\nu\|_{L^{\infty}(0, T ; H) \cap L^{2}(0, T ; V)}+\|\psi\|_{H^{1}(0, T ; H) \cap L^{\infty}(0, T ; V)}+\|\rho\|_{L^{\infty}(0, T ; H) \cap L^{2}(0, T ; V)} \\
& \quad \leq c\left\|\overline{\mathbf{u}}^{1}-\overline{\mathbf{u}}^{2}\right\|_{L^{2}(0, T ; H)^{2}}\|\mathbf{h}\|_{L^{2}(0, T ; H)^{2}}\|\mathbf{k}\|_{L^{2}(0, T ; H)^{2}} .
\end{aligned}
$$

SECOND ESTimate: The above estimate entails that the norm of $\partial_{t} \psi$ in $L^{2}(0, T ; H)$ is bounded by the expression on the right-hand side. Thus, in equation (4.107) the term $\partial_{t} \psi$ can be absorbed on the right-hand side. A straightforward computation, which may be left to the reader, shows that the entire right-hand side is bounded in $L^{2}(0, T ; H)$ by the same expression. Hence, we can infer from the elliptic regularity theory that also

$$
\|\psi\|_{L^{2}\left(0, T ; W_{0}\right)} \leq c\left\|\overline{\mathbf{u}}^{1}-\overline{\mathbf{u}}^{2}\right\|_{L^{2}(0, T ; H)^{2}}\|\mathbf{h}\|_{L^{2}(0, T ; H)^{2}}\|\mathbf{k}\|_{L^{2}(0, T ; H)^{2}} .
$$

This concludes the proof of the assertion.

\section{FIRST-ORDER NECESSARY OPTIMALITY CONDITIONS}

We now derive first-order necessary optimality conditions. By the well-known characterization for differentiable maps on convex sets, it holds (see, e.g., [47]) that

$$
D \mathcal{J}_{\text {red }}(\overline{\mathbf{u}})(\mathbf{u}-\overline{\mathbf{u}}) \geq 0 \quad \forall \mathbf{u} \in \mathcal{U}_{\mathrm{ad}},
$$

where $D \mathcal{J}_{\text {red }}$ denotes the derivative of the reduced cost functional given by

$$
\mathcal{J}_{\text {red }}(\mathbf{u}):=\mathcal{J}(\mathcal{S}(\mathbf{u}), \mathbf{u})
$$

Therefore, using Theorem 4.3 and the chain rule, we have the following result:

Theorem 5.1. Suppose that (W1), (S1)-(S4), (C1)-(C3), as well as (2.33)-(2.34), are fulfilled. Moreover, let $\overline{\mathbf{u}}$ be an optimal control for (eP) with corresponding state $(\bar{\mu}, \bar{\varphi}, \bar{\sigma})$. Then it holds the variational inequality

$$
b_{1} \int_{Q}\left(\bar{\varphi}-\widehat{\varphi}_{Q}\right) \xi+b_{2} \int_{\Omega}\left(\bar{\varphi}(T)-\widehat{\varphi}_{\Omega}\right) \xi(T)+b_{0} \int_{Q} \overline{\mathbf{u}} \cdot(\mathbf{u}-\overline{\mathbf{u}}) \geq 0 \quad \forall \mathbf{u} \in \mathcal{U}_{\mathrm{ad}},
$$

where $(\eta, \xi, \theta)$ denotes the unique solution to the linearized system obtained from Lemma 4.1 with $\mathbf{h}=\mathbf{u}-\overline{\mathbf{u}}$ and $\lambda_{1}=\lambda_{2}=1, \lambda_{3}=\lambda_{4}=0$.

As usual, we simplify (5.3) by means of the adjoint state variables $(p, q, r)$, which are defined as the solution triple to the adjoint system whose strong form is given by the backward-in-time parabolic system

$$
\begin{array}{ll}
-\partial_{t} p-\beta \partial_{t} q-\Delta q+\chi \Delta r+F^{\prime \prime}(\bar{\varphi}) q+\mathbb{h}^{\prime}(\bar{\varphi}) \bar{u}_{1} p & \\
\quad-P^{\prime}(\bar{\varphi})(\bar{\sigma}+\chi(1-\bar{\varphi})-\bar{\mu})(p-r)+\chi P(\bar{\varphi})(p-r)=b_{1}\left(\bar{\varphi}-\widehat{\varphi}_{Q}\right) & \text { in } Q, \\
-\alpha \partial_{t} p-\Delta p-q+P(\bar{\varphi})(p-r)=0 & \text { in } Q, \\
-\partial_{t} r-\Delta r-\chi q-P(\bar{\varphi})(p-r)=0 & \text { in } Q, \\
\partial_{\mathbf{n}} p=\partial_{\mathbf{n}} q=\partial_{\mathbf{n}} r=0 & \text { on } \Sigma, \\
(p+\beta q)(T)=b_{2}\left(\bar{\varphi}(T)-\widehat{\varphi}_{\Omega}\right), \quad \alpha p(T)=0, \quad r(T)=0 & \text { in } \Omega .
\end{array}
$$


Let us point out that since the terminal condition $(p+\beta q)(T)$ prescribes a final datum in $L^{2}(\Omega)(c f$. (C2)), it is clear that the first equation (5.4) has to be considered in a weak sense. Here is the corresponding well-posedness result.

Theorem 5.2 (Well-posedness of the adjoint system). Suppose that (W1), (S1)-(S4), (C1)-(C3), and (2.33)-(2.34) hold. Then the adjoint system (5.4)-(5.8) admits a unique solution $(p, q, r)$ in the sense that

$$
\begin{aligned}
p+\beta q & \in H^{1}\left(0, T ; V^{*}\right), \\
p & \in H^{1}(0, T ; H) \cap L^{\infty}(0, T ; V) \cap L^{2}\left(0, T ; W_{0}\right) \cap L^{\infty}(Q), \\
q & \in L^{\infty}(0, T ; H) \cap L^{2}(0, T ; V), \\
r & \in H^{1}(0, T ; H) \cap L^{\infty}(0, T ; V) \cap L^{2}\left(0, T ; W_{0}\right) \cap L^{\infty}(Q),
\end{aligned}
$$

where $(p, q, r)$ satisfies

$$
\begin{aligned}
& -\left\langle\partial_{t}(p+\beta q), v\right\rangle+\int_{\Omega} \nabla q \cdot \nabla v-\chi \int_{\Omega} \nabla r \cdot \nabla v+\int_{\Omega} F^{\prime \prime}(\bar{\varphi}) q v+\int_{\Omega} \mathbb{h}^{\prime}(\bar{\varphi}) \bar{u}_{1} p v \\
& -\int_{\Omega} P^{\prime}(\bar{\varphi})(\bar{\sigma}+\chi(1-\bar{\varphi})-\bar{\mu})(p-r) v+\chi \int_{\Omega} P(\bar{\varphi})(p-r) v=b_{1} \int_{\Omega}\left(\bar{\varphi}-\widehat{\varphi}_{Q}\right) v, \\
& -\left\langle\alpha \partial_{t} p, v\right\rangle+\int_{\Omega} \nabla p \cdot \nabla v-\int_{\Omega} q v+\int_{\Omega} P(\bar{\varphi})(p-r) v=0, \\
& -\left\langle\partial_{t} r, v\right\rangle+\int_{\Omega} \nabla r \cdot \nabla v-\chi \int_{\Omega} q v-\int_{\Omega} P(\bar{\varphi})(p-r) v=0,
\end{aligned}
$$

for every $v \in V$ and almost every $t \in(0, T)$, as well as the terminal conditions

$$
(p+\beta q)(T)=b_{2}\left(\bar{\varphi}(T)-\widehat{\varphi}_{\Omega}\right), \quad \alpha p(T)=0, \quad r(T)=0, \quad \text { a.e. in } \Omega .
$$

Remark 5.3. Before entering the proof of the above theorem, let us point out that the regularity conditions on the solution imply that both $p+\beta q$ and $q$ are in $H^{1}\left(0, T ; V^{*}\right) \cap C^{0}([0, T] ; H)$, whence all terminal conditions make sense. About that, we observe that the first condition may be rewritten just in terms of $q$ as $\beta q(T)=$ $b_{2}\left(\bar{\varphi}(T)-\widehat{\varphi}_{\Omega}\right)$.

Proof. For brevity, we again argue formally, thus avoiding the introduction of approximation schemes like in the proof of Lemma 4.1 and just providing the relevant a priori estimates. Moreover, let us notice that the adjoint system (5.4)-(5.8) is linear, so that the uniqueness part also follows from standard arguments as a consequence of the following estimates.

FiRST ESTIMATE: First, we add to both sides of (5.5) the term $p$. We then multiply (5.4) by $q$, the new (5.5) by $-\partial_{t} p$, and (5.6) by $\chi^{2} r$, add the resulting equalities, and integrate over $Q_{t}^{T}=\Omega \times(t, T)$ and by parts to obtain that

$$
\begin{aligned}
\frac{\beta}{2}\|q(t)\|^{2}+\int_{Q_{t}^{T}}|\nabla q|^{2}+\alpha \int_{Q_{t}^{T}}\left|\partial_{t} p\right|^{2}+\frac{1}{2}\|p(t)\|_{V}^{2}+\frac{\chi^{2}}{2}\|r(t)\|^{2}+\chi^{2} \int_{Q_{t}^{T}}|\nabla r|^{2} \\
=\frac{\beta}{2}\|q(T)\|^{2}+b_{1} \int_{Q_{t}^{T}}\left(\bar{\varphi}-\widehat{\varphi}_{Q}\right) q+\chi \int_{Q_{t}^{T}} \nabla r \cdot \nabla q-\int_{Q_{t}^{T}} F^{\prime \prime}(\bar{\varphi})|q|^{2} \\
\quad-\int_{Q_{t}^{T}} \mathbb{h}^{\prime}(\bar{\varphi}) \bar{u}_{1} p q+\int_{Q_{t}^{T}} P^{\prime}(\bar{\varphi})(\bar{\sigma}+\chi(1-\bar{\varphi})-\bar{\mu})(p-r) q-\chi \int_{Q_{t}^{T}} P(\bar{\varphi})(p-r) q
\end{aligned}
$$




$$
+\int_{Q_{t}^{T}} P(\bar{\varphi})(p-r) \partial_{t} p-\int_{Q_{t}^{T}} p \partial_{t} p+\chi^{3} \int_{Q_{t}^{T}} q r+\chi^{2} \int_{Q_{t}^{T}} P(\bar{\varphi})(p-r) r=: \sum_{i=1}^{11} I_{i}
$$

We estimate the terms on the right-hand side individually. The first summand is bounded by a constant, due to the terminal conditions (5.8) and the assumptions (C1)-(C2). For the other terms on the same line, we have that

$$
I_{2}+I_{3}+I_{4} \leq c \int_{Q_{t}^{T}}\left(|q|^{2}+1\right)+\frac{\chi^{2}}{2} \int_{Q_{t}^{T}}|\nabla r|^{2}+\frac{1}{2} \int_{Q_{t}^{T}}|\nabla q|^{2}
$$

by virtue of Young's inequality, the assumptions (C1)-(C2), and the separation property (2.39). Using the Young and Hölder inequalities, we bound the integrals in the second line of the right-hand side by

$$
\begin{aligned}
I_{5}+I_{6}+I_{7} \leq & c \int_{Q_{t}^{T}}\left(|p|^{2}+|q|^{2}\right)+c \int_{t}^{T}\left(\|\bar{\sigma}\|_{\infty}+\|\bar{\varphi}\|_{\infty}+\|\bar{\mu}\|_{\infty}+1\right)(\|p\|+\|q\|)\|q\| \mathrm{d} s \\
& +c \int_{Q_{t}^{T}}\left(|p|^{2}+|q|^{2}+|r|^{2}\right) \leq c \int_{Q_{t}^{T}}\left(|p|^{2}+|q|^{2}+|r|^{2}\right),
\end{aligned}
$$

where we also owe to the boundedness of $P, P^{\prime}$ and $\bar{u}_{1}$, and to the fact that $(\bar{\mu}, \bar{\varphi}, \bar{\sigma})$, as a solution to (1.2)-(1.6) in the sense of Theorem 2.3, satisfies (2.38). Finally, the terms on the last line of the right-hand side can be easily bounded by means of Young's inequality, namely,

$$
I_{8}+I_{9}+I_{10}+I_{11} \leq \frac{\alpha}{2} \int_{Q_{t}^{T}}\left|\partial_{t} p\right|^{2}+c \int_{Q_{t}^{T}}\left(|p|^{2}+|q|^{2}+|r|^{2}\right)
$$

Now, we combine the above estimates and invoke Gronwall's lemma to infer that

$$
\|p\|_{H^{1}(0, T ; H) \cap L^{\infty}(0, T ; V)}+\|q\|_{L^{\infty}(0, T ; H) \cap L^{2}(0, T ; V)}+\|r\|_{L^{\infty}(0, T ; H) \cap L^{2}(0, T ; V)} \leq c .
$$

SECOND ESTIMATE: We can now rewrite equation (5.6) as a parabolic equation with the source term $f_{r}$ : $=$ $\chi_{q}+P(\bar{\varphi})(p-r)$, which is bounded in $L^{\infty}(0, T ; H)$ due to the above estimate. It is then a standard matter to infer that

$$
\|r\|_{H^{1}(0, T ; H) \cap L^{\infty}(0, T ; V) \cap L^{2}\left(0, T ; W_{0}\right)} \leq c .
$$

In addition, since $r(T)=0 \in L^{\infty}(\Omega)$, we can apply the regularity result ([36], Thm. 7.1, p. 181) to infer that $\|r\|_{L^{\infty}(Q)} \leq c$ as well.

ThiRD ESTIMATE: From equation (5.5) and the parabolic regularity theory, similarly we recover that

$$
\|p\|_{L^{2}\left(0, T ; W_{0}\right) \cap L^{\infty}(Q)} \leq c .
$$

FOURTH ESTIMATE: Finally, comparison in equation (5.4), along with the above estimates, produces that

$$
\left\|\partial_{t}(p+\beta q)\right\|_{L^{2}\left(0, T ; V^{*}\right)} \leq c,
$$

and this further estimate concludes the proof. 
It is then a standard matter to use the adjoint variables to simplify the first-order necessary conditions obtained in Theorem 5.1.

Theorem 5.4. Assume that (W1), (S1)-(S4), (C1)-(C3), and (2.33)-(2.34) are fulfilled, and let $\overline{\mathbf{u}} \in \mathcal{U}_{\mathrm{ad}}$ be an optimal control for (eP) with corresponding state $(\bar{\mu}, \bar{\varphi}, \bar{\sigma})$. Then, setting

$$
\mathbf{d}(x, t):=(-\mathbb{h}(\bar{\varphi}(x, t)) p(x, t), r(x, t)), \quad \text { for a.e. }(x, t) \in Q,
$$

we have that

$$
\int_{Q}\left(\mathbf{d}+b_{0} \overline{\mathbf{u}}\right) \cdot(\mathbf{u}-\overline{\mathbf{u}}) \geq 0 \quad \forall \mathbf{u} \in \mathcal{U}_{\mathrm{ad}}
$$

Moreover, $\overline{\mathbf{u}}$ is the $L^{2}(0, T ; H)^{2}$-orthogonal projection of $-b_{0}^{-1} \mathbf{d}$ onto $\mathcal{U}_{\mathrm{ad}}$.

Proof. By comparing the variational inequalities (5.3) and (5.9), it follows that it suffices to show the identity

$$
-\int_{Q} \mathfrak{h}(\bar{\varphi}) h_{1} p+\int_{Q} h_{2} r=b_{1} \int_{Q}\left(\bar{\varphi}-\widehat{\varphi}_{Q}\right) \xi+b_{2} \int_{\Omega}\left(\bar{\varphi}(T)-\widehat{\varphi}_{\Omega}\right) \xi(T),
$$

with the choices $h_{1}=u_{1}-\bar{u}_{1}$ and $h_{2}=u_{2}-\bar{u}_{2}$, where $(\eta, \xi, \theta)$ and $(p, q, r)$ denote the unique solutions to the linearized system and to the adjoint system obtained from Lemma 4.1 with $\lambda_{1}=\lambda_{2}=1$ and $\lambda_{3}=\lambda_{4}=0$, and Theorem 5.2, respectively.

In this direction, we multiply the linearized system, written with $(\mu, \varphi, \sigma)=(\eta, \xi, \theta)$, by $p, q$ and $r$, in this order. Then we add the obtained equalities and integrate over $Q$ and by parts to obtain that

$$
\begin{aligned}
0= & \int_{0}^{T}\left\langle-\partial_{t}(p+\beta q), \xi\right\rangle \mathrm{d} t+\int_{Q} \nabla \xi \cdot \nabla q \\
& +\int_{Q} \xi\left[\chi \Delta r+F^{\prime \prime}(\bar{\varphi}) q+\mathbb{h}^{\prime}(\bar{\varphi}) \bar{u}_{1} p\right. \\
& \left.\quad-P^{\prime}(\bar{\varphi})(\bar{\sigma}+\chi(1-\bar{\varphi})-\bar{\mu})(p-r)+\chi P(\bar{\varphi})(p-r)\right] \\
& +\int_{Q} \eta\left[-\alpha \partial_{t} p-\Delta p-q+P(\bar{\varphi})(p-r)\right] \\
& +\int_{Q} \theta\left[-\partial_{t} r-\Delta r-\chi q-P(\bar{\varphi})(p-r)\right] \\
& +\int_{\Omega}[\alpha p(T) \eta(T)+(p+\beta q)(T) \xi(T)+r(T) \theta(T)] \\
& +\int_{Q}\left(\mathbb{h}(\bar{\varphi}) h_{1} p-h_{2} r\right) .
\end{aligned}
$$

Now, we use the weak form of (5.4), as well as (5.5)-(5.8), to deduce that the above equality reduces to (5.10), which concludes the proof.

\section{SECOND-ORDER SUFFICIENT OPTIMALITY CONDITIONS}

We now establish second-order sufficient optimality conditions. Since the control-to-state mapping $\mathcal{S}$ is only known to be Fréchet differentiable on $\mathcal{U}$, we are faced with the so-called "two-norm discrepancy" (see also [47], Sec. 4.10.2). In order to overcome this difficulty, we follow the approach taken in Chapter 5 of [47]. Since many 
of the arguments developed here are rather similar to those employed in [47], we can afford to be sketchy here. For full details, we refer the reader to [47], noting that there the case of one control variable is treated while in our case we have to deal with a pair of controls. In order to simplify the analysis somewhat, we now make an additional assumption.

It holds $b_{2}=0$.

Notice that under the assumption (C4) we have a zero terminal condition for $p+\beta q$ in (5.8). This easily leads to the conclusion that we have the additional regularity $q \in Z$, which, in turn, means that the adjoint system is satisfied in the strong form (5.4)-(5.8).

By virtue of (C4), we readily infer that for every $((\mu, \varphi, \sigma), \mathbf{u}) \in\left(C^{0}([0, T] ; H)\right)^{3} \times \mathcal{U}$ and $\mathbf{v}=\left(v_{1}, v_{2}, v_{3}\right), \mathbf{w}=$ $\left(w_{1}, w_{2}, w_{3}\right)$ such that $(\mathbf{v}, \mathbf{h}),(\mathbf{w}, \mathbf{k}) \in\left(C^{0}([0, T] ; H)\right)^{3} \times \mathcal{U}$ we have

$$
D^{2} \mathcal{J}((\mu, \varphi, \sigma), \mathbf{u})((\mathbf{v}, \mathbf{h}),(\mathbf{w}, \mathbf{k}))=b_{1} \int_{Q} v_{2} w_{2}+b_{0} \int_{Q} \mathbf{h} \cdot \mathbf{k} .
$$

Using Theorem 4.7 along with the above expression, we can now derive the second-order derivative of the reduced cost functional $\partial_{\text {red }}$. Namely, for a fixed control $\overline{\mathbf{u}}$ we find that

$$
\begin{aligned}
D^{2} \mathcal{\partial}_{\text {red }}(\overline{\mathbf{u}})(\mathbf{h}, \mathbf{k})= & D_{(\mu, \varphi, \sigma)} \mathcal{\partial}((\bar{\mu}, \bar{\varphi}, \bar{\sigma}), \overline{\mathbf{u}})(\nu, \psi, \rho) \\
& +D^{2} \mathcal{J}((\bar{\mu}, \bar{\varphi}, \bar{\sigma}), \overline{\mathbf{u}})\left(\left(\left(\eta^{\mathbf{h}}, \xi^{\mathbf{h}}, \theta^{\mathbf{h}}\right), \mathbf{h}\right),\left(\left(\eta^{\mathbf{k}}, \xi^{\mathbf{k}}, \theta^{\mathbf{k}}\right), \mathbf{k}\right)\right),
\end{aligned}
$$

where $\left(\eta^{\mathbf{h}}, \xi^{\mathbf{h}}, \theta^{\mathbf{h}}\right),\left(\eta^{\mathbf{k}}, \xi^{\mathbf{k}}, \theta^{\mathbf{k}}\right)$, and $(\nu, \psi, \rho)$ stand for the unique corresponding solutions to the linearized system associated with $\mathbf{h}$ and $\mathbf{k}$, and to the bilinearized system, respectively. From the definition of the cost functional (1.1) (recall that now $b_{2}=0$ ), we readily infer that

$$
D_{(\mu, \varphi, \sigma)} \mathcal{\partial}((\bar{\mu}, \bar{\varphi}, \bar{\sigma}), \overline{\mathbf{u}})(\nu, \psi, \rho)=b_{1} \int_{Q}\left(\bar{\varphi}-\widehat{\varphi}_{Q}\right) \psi
$$

We now claim that

$$
\begin{aligned}
b_{1} \int_{Q}\left(\bar{\varphi}-\widehat{\varphi}_{Q}\right) \psi=\int_{Q} & {\left[P^{\prime}(\bar{\varphi}) \xi^{\mathbf{k}}\left(\theta^{\mathbf{h}}-\chi \xi^{\mathbf{h}}-\eta^{\mathbf{h}}\right)(p-r)\right.} \\
& +P^{\prime \prime}(\bar{\varphi}) \xi^{\mathbf{k}} \xi^{\mathbf{h}}(\bar{\sigma}+\chi(1-\bar{\varphi})-\bar{\mu})(p-r) \\
& +P^{\prime}(\bar{\varphi}) \xi^{\mathbf{h}}\left(\theta^{\mathbf{k}}-\chi \xi^{\mathbf{k}}-\eta^{\mathbf{k}}\right)(p-r)-\mathbb{h}^{\prime \prime}(\bar{\varphi}) \xi^{\mathbf{k}} \xi^{\mathbf{h}} \bar{u}_{1} p \\
& \left.-\mathbb{h}^{\prime}(\bar{\varphi}) \xi^{\mathbf{h}} k_{1} p-\mathbb{h}^{\prime}(\bar{\varphi}) \xi^{\mathbf{k}} h_{1} p-F^{(3)}(\bar{\varphi}) \xi^{\mathbf{h}} \xi^{\mathbf{k}} q\right]
\end{aligned}
$$

To prove this claim, we multiply (4.78) by $p,(4.79)$ by $q$, (4.80) by $r$, add the resulting equalities, and integrate over $Q$, to obtain that

$$
\begin{aligned}
0= & \int_{Q} p\left[\alpha \partial_{t} \nu+\partial_{t} \psi-\Delta \nu-g_{1}-g_{2}\right] \\
& +\int_{Q} q\left[\beta \partial_{t} \psi-\Delta \psi-\nu-\chi \rho+F^{\prime \prime}(\bar{\varphi}) \psi+F^{(3)}(\bar{\varphi}) \xi^{\mathbf{h}} \xi^{\mathbf{k}}\right] \\
& +\int_{Q} r\left[\partial_{t} \rho-\Delta \rho+\chi \Delta \psi+g_{1}\right]
\end{aligned}
$$


with the functions $g_{1}$ and $g_{2}$ defined in (4.83)-(4.84). Then, we integrate by parts and make use of the initial and terminal conditions (4.82) and (5.8) to find that

$$
\begin{aligned}
0= & \int_{Q} \nu\left[-\alpha \partial_{t} p-\Delta p-q+P(\bar{\varphi})(p-r)\right] \\
& +\int_{Q} \psi\left[-\partial_{t} p-\beta \partial_{t} q-\Delta q+\chi \Delta r+F^{\prime \prime}(\bar{\varphi}) q+\mathbb{h}^{\prime}(\bar{\varphi}) \bar{u}_{1} p\right. \\
& \left.\quad-P^{\prime}(\bar{\varphi})(\bar{\sigma}+\chi(1-\bar{\varphi})-\bar{\mu})(p-r)+\chi P(\bar{\varphi})(p-r)\right] \\
& +\int_{Q} \rho\left[-\partial_{t} r-\Delta r-\chi q-P(\bar{\varphi})(p-r)\right] \\
+ & \int_{Q}\left[-P^{\prime}(\bar{\varphi}) \xi^{\mathbf{k}}\left(\theta^{\mathbf{h}}-\chi \xi^{\mathbf{h}}-\eta^{\mathbf{h}}\right)(p-r)\right. \\
& \quad-P^{\prime \prime}(\bar{\varphi}) \xi^{\mathbf{k}} \xi^{\mathbf{h}}(\bar{\sigma}+\chi(1-\bar{\varphi})-\bar{\mu})(p-r) \\
& \quad-P^{\prime}(\bar{\varphi}) \xi^{\mathbf{h}}\left(\theta^{\mathbf{k}}-\chi \xi^{\mathbf{k}}-\eta^{\mathbf{k}}\right)(p-r)+\mathbb{h}^{\prime \prime}(\bar{\varphi}) \xi^{\mathbf{k}} \xi^{\mathbf{h}} \bar{u}_{1} p \\
& \left.+\mathbb{h}^{\prime}(\bar{\varphi}) \xi^{\mathbf{h}} k_{1} p+\mathbb{h}^{\prime}(\bar{\varphi}) \xi^{\mathbf{k}} h_{1} p+F^{(3)}(\bar{\varphi}) \xi^{\mathbf{h}} \xi^{\mathbf{k}} q\right],
\end{aligned}
$$

whence the claim follows by using the adjoint system. From this characterization, along with (6.2) and (6.3), we conclude that

$$
\begin{aligned}
& D^{2} \partial_{\mathrm{red}}(\overline{\mathbf{u}})(\mathbf{h}, \mathbf{h})=b_{0}\|\mathbf{h}\|_{L^{2}(0, T ; H)^{2}}^{2}+\int_{Q} 2 P^{\prime}(\bar{\varphi}) \xi^{\mathbf{h}}\left(\theta^{\mathbf{h}}-\eta^{\mathbf{h}}\right)(p-r)-\int_{Q} 2 \mathbb{h}^{\prime}(\bar{\varphi}) \xi^{\mathbf{h}} h_{1} p \\
& \quad+\int_{Q}\left(b_{1}-P^{\prime \prime}(\bar{\varphi})(\bar{\sigma}+\chi(1-\bar{\varphi})-\bar{\mu})(p-r)-2 \chi P^{\prime}(\bar{\varphi})(p-r)\right. \\
& \left.\quad+\mathbb{h}^{\prime \prime}(\bar{\varphi}) \bar{u}_{1} p+F^{(3)}(\bar{\varphi}) q\right)\left|\xi^{\mathbf{h}}\right|^{2}
\end{aligned}
$$

This explicit expression for the second-order derivative of $\mathcal{J}_{\text {red }}$ allows us to establish sufficient conditions for optimality of $\overline{\mathbf{u}}$. We aim at showing that, under suitable assumptions, $D^{2} \mathcal{J}_{\text {red }}(\overline{\mathbf{u}})$ is a positive definite operator on a suitable subset of $L^{2}(0, T ; H)^{2}$, meaning that for any admissible increment $\mathbf{h}$ it holds that

$$
D^{2} \mathcal{J}_{\text {red }}(\overline{\mathbf{u}})(\mathbf{h}, \mathbf{h})>0 .
$$

However, (6.6) is rather restrictive as we need such a condition just along some suitable directions. To this end, for every $\tau>0$, we introduce the sets of strongly active constraints,

$$
\begin{aligned}
& A_{\tau}^{1}(\overline{\mathbf{u}}):=\left\{(x, t) \in Q:\left|-\mathbb{h}(\bar{\varphi}(x, t)) p(x, t)+b_{0} \bar{u}_{1}(x, t)\right|>\tau\right\}, \\
& A_{\tau}^{2}(\overline{\mathbf{u}}):=\left\{(x, t) \in Q:\left|r(x, t)+b_{0} \bar{u}_{2}(x, t)\right|>\tau\right\} .
\end{aligned}
$$

Moreover, for any increment $\mathbf{h}=\left(h_{1}, h_{2}\right) \in \mathcal{U}$ we introduce the componentwise conditions

$$
\begin{gathered}
h_{1}(x, t) \begin{cases}=0 & \text { if }(x, t) \in A_{\tau}^{1}(\overline{\mathbf{u}}), \\
\geq 0 & \text { if } \bar{u}_{1}(x, t)=\underline{u}_{1}(x, t) \text { and }(x, t) \notin A_{\tau}^{1}(\overline{\mathbf{u}}), \\
\leq 0 & \text { if } \bar{u}_{1}(x, t)=\widehat{u}_{1}(x, t) \text { and }(x, t) \notin A_{\tau}^{1}(\overline{\mathbf{u}}),\end{cases} \\
h_{2}(x, t) \begin{cases}=0 & \text { if }(x, t) \in A_{\tau}^{2}(\overline{\mathbf{u}}), \\
\geq 0 & \text { if } \bar{u}_{2}(x, t)=\underline{u}_{2}(x, t) \text { and }(x, t) \notin A_{\tau}^{2}(\overline{\mathbf{u}}), \\
\leq 0 & \text { if } \bar{u}_{2}(x, t)=\widehat{u}_{2}(x, t) \text { and }(x, t) \notin A_{\tau}^{2}(\overline{\mathbf{u}}),\end{cases}
\end{gathered}
$$


and define the associated $\tau$-critical cone by

$$
\mathcal{C}_{\tau}(\overline{\mathbf{u}}):=\left\{\mathbf{h}=\left(h_{1}, h_{2}\right) \in \mathcal{U}: h_{1} \text { satisfies (6.7) and } h_{2} \text { satisfies (6.8) a.e. in } Q\right\} .
$$

The second-order sufficient condition for optimality then reads as follows:

$$
\exists \delta, \tau>0: D^{2} \mathcal{J}_{\text {red }}(\overline{\mathbf{u}})(\mathbf{h}, \mathbf{h}) \geq \delta\|\mathbf{h}\|_{L^{2}(0, T ; H)^{2}}^{2} \quad \forall \mathbf{h} \in \mathcal{C}_{\tau}(\overline{\mathbf{u}}),
$$

where $D^{2} \mathcal{J}_{\text {red }}(\overline{\mathbf{u}})(\mathbf{h}, \mathbf{h})$ is given by $(6.5)$ with the choices $(\bar{\mu}, \bar{\varphi}, \bar{\sigma})=\mathcal{S}(\overline{\mathbf{u}}),\left(\eta^{\mathbf{h}}, \xi^{\mathbf{h}}, \theta^{\mathbf{h}}\right)=D \mathcal{S}(\overline{\mathbf{u}})(\mathbf{h})$, and the corresponding adjoint variables $(p, q, r)$. We have the following result.

Theorem 6.1. Assume that (W1), (S1)-(S4), (C1)-(C4), as well as (2.33)-(2.34) are fulfilled. Let $\overline{\mathbf{u}} \in \mathcal{U}_{\mathrm{ad}}$ be an admissible control which satisfies (5.3) and (6.10) with the corresponding state $(\bar{\mu}, \bar{\varphi}, \bar{\sigma})$ and adjoint variables $(p, q, r)$, as obtained from Theorem 2.3 and 5.2, respectively. Then there exist positive constants $\varepsilon_{1}, \varepsilon_{2}$ such that

$$
\mathcal{J}_{\text {red }}(\mathbf{u}) \geq \mathcal{J}_{\text {red }}(\overline{\mathbf{u}})+\varepsilon_{1}\|\mathbf{u}-\overline{\mathbf{u}}\|_{L^{2}(0, T ; H)^{2}}^{2} \quad \text { for every } \quad \mathbf{u} \in \mathcal{U} \quad \text { such that }\|\mathbf{u}-\overline{\mathbf{u}}\|_{\mathfrak{U}} \leq \varepsilon_{2} .
$$

In particular, it follows that $\overline{\mathbf{u}}$ is locally optimal for $(\mathrm{CP})$ in the sense of $\mathcal{U}$.

For the proof we follow the line of argumentation employed in the proof of Theorem 5.17 in [47], where in our case we deal with a system of parabolic equations and a pair of controls, with state and control nonlinearly coupled. However, the techniques used in [47] can straightforwardly be adapted to our more complicated situation. We therefore merely sketch the arguments.

Proof. Given an arbitrary $\mathbf{u} \in \mathfrak{U}_{\mathrm{ad}}$, we infer from Taylor's theorem with integral remainder that

$$
\mathcal{J}_{\text {red }}(\mathbf{u})-\mathcal{J}_{\text {red }}(\overline{\mathbf{u}})=D \mathcal{J}_{\text {red }}(\overline{\mathbf{u}})(\mathbf{v})+\frac{1}{2} D^{2} \mathcal{\partial}_{\text {red }}(\overline{\mathbf{u}})(\mathbf{v}, \mathbf{v})+R^{\mathcal{J}_{\text {red }}}(\mathbf{u}, \overline{\mathbf{u}}),
$$

where we have set $\mathbf{v}=\mathbf{u}-\overline{\mathbf{u}}$, and where the remainder $R^{\mathfrak{J}_{\text {red }}}$ is given by

$$
R^{\mathcal{J}_{\text {red }}}(\mathbf{u}, \overline{\mathbf{u}})=\int_{0}^{1}(1-s)\left(D^{2} \mathcal{J}_{\text {red }}(\overline{\mathbf{u}}+s \mathbf{v})-D^{2} \mathcal{\partial}_{\text {red }}(\overline{\mathbf{u}})\right)(\mathbf{v}, \mathbf{v}) \mathrm{d} s .
$$

To estimate $\left(D^{2} \mathcal{\partial}_{\text {red }}(\overline{\mathbf{u}}+s \mathbf{v})-D^{2} \mathcal{\partial}_{\text {red }}(\overline{\mathbf{u}})\right)(\mathbf{v}, \mathbf{v})$, we set

$$
\begin{aligned}
\left(\mu^{s}, \varphi^{s}, \sigma^{s}\right) & =\mathcal{S}(\overline{\mathbf{u}}+s \mathbf{v}), \quad(\eta, \xi, \theta)=D \mathcal{S}(\overline{\mathbf{u}})(\mathbf{v}), \quad\left(\eta^{s}, \xi^{s}, \theta^{s}\right)=D \mathcal{S}(\overline{\mathbf{u}}+s \mathbf{v})(\mathbf{v}) \\
(\nu, \psi, \rho) & =D^{2} \mathcal{S}(\overline{\mathbf{u}})(\mathbf{v}, \mathbf{v}), \quad\left(\nu^{s}, \psi^{s}, \rho^{s}\right)=D^{2} \mathcal{S}(\overline{\mathbf{u}}+s \mathbf{v})(\mathbf{v}, \mathbf{v})
\end{aligned}
$$

By arguing along the lines of the proof ([47], Thm. 5.17), we find that it suffices to show that

$$
\frac{\left|R^{J_{\mathrm{red}}}(\mathbf{u}, \overline{\mathbf{u}})\right|}{\|\mathbf{u}-\overline{\mathbf{u}}\|_{L^{2}(0, T ; H)^{2}}^{2}} \longrightarrow 0 \quad \text { as } \quad\|\mathbf{u}-\overline{\mathbf{u}}\|_{\mathfrak{U}} \rightarrow 0
$$

Using (6.3), we see that

$$
\begin{aligned}
& D_{(\mu, \varphi, \sigma)} \mathcal{J}\left(\left(\mu^{s}, \varphi^{s}, \sigma^{s}\right), \overline{\mathbf{u}}+s \mathbf{v}\right)\left(\nu^{s}, \psi^{s}, \rho^{s}\right)-D_{(\mu, \varphi, \sigma)} \mathcal{J}((\bar{\mu}, \bar{\varphi}, \bar{\sigma}), \overline{\mathbf{u}})(\nu, \psi, \rho) \\
& \quad=b_{1} \int_{Q}\left(\varphi^{s}-\bar{\varphi}\right) \psi+b_{1} \int_{Q}\left(\varphi^{s}-\widehat{\varphi}_{Q}\right)\left(\psi^{s}-\psi\right)=: I_{1},
\end{aligned}
$$


and from (6.1)-(6.2) we obtain that

$$
\begin{aligned}
& D^{2} \mathcal{J}\left(\left(\mu^{s}, \varphi^{s}, \sigma^{s}\right), \overline{\mathbf{u}}+s \mathbf{v}\right)\left(\left(\left(\eta^{s}, \xi^{s}, \theta^{s}\right), \mathbf{v}\right),\left(\left(\eta^{s}, \xi^{s}, \theta^{s}\right), \mathbf{v}\right)\right) \\
& \quad-D^{2} \mathcal{J}((\mu, \varphi, \sigma), \overline{\mathbf{u}})(((\eta, \xi, \theta), \mathbf{v}),((\eta, \xi, \theta), \mathbf{v}))=b_{1} \int_{Q}\left(\xi^{s}+\xi\right)\left(\xi^{s}-\xi\right)=: I_{2} .
\end{aligned}
$$

It then readily follows from the Cauchy-Schwarz inequality, and the stability estimates (2.14), (4.65) and (4.104), that

$$
\begin{aligned}
I_{1} & \leq b_{1}\left(\left\|\varphi^{s}-\bar{\varphi}\right\|_{L^{2}(0, T ; H)}\|\psi\|_{L^{2}(0, T ; H)}+\left\|\varphi^{s}-\widehat{\varphi}_{Q}\right\|_{L^{2}(0, T ; H)}\left\|\psi^{s}-\psi\right\|_{L^{2}(0, T ; H)}\right) \\
& \leq c s\|\mathbf{v}\|_{L^{2}(0, T ; H)^{2}}^{3} .
\end{aligned}
$$

Moreover, owing to (C2), Theorem 4.3, and to the stability estimate (4.65),

$$
I_{2} \leq b_{1}\left(\left\|\xi^{s}+\xi\right\|_{L^{2}(0, T ; H)}\left\|\xi^{s}-\xi\right\|_{L^{2}(0, T ; H)}\right) \leq c s\|\mathbf{v}\|_{L^{2}(0, T ; H)^{2}}^{3} .
$$

We thus can conclude that

$$
\left|R^{\mathcal{I}_{\mathrm{red}}}(\mathbf{u}, \overline{\mathbf{u}})\right| \leq c \int_{0}^{1}(1-s) s\|\mathbf{v}\|_{L^{2}(0, T ; H)^{2}}^{3} \mathrm{~d} s \leq c\|\mathbf{v}\| u\|\mathbf{v}\|_{L^{2}(0, T ; H)^{2}}^{2},
$$

so that (6.11) directly follows. With this, the proof can be completed by adapting the argumentation of [47] correspondingly to our situation.

\section{Conclusions}

We investigate a distributed optimal control problem for a tumor growth model of viscous Cahn-Hilliard type with source term including chemotaxis effects and possibly singular potentials. Similar systems have already been investigated from the viewpoint of classical optimal control theory, obtaining the existence of optimal controls and first-order necessary conditions for optimality. This paper aims at contributing to this common effort by providing some mathematical results on second-order optimality conditions: indeed, this subject is widely open for similar systems. In this direction, we are just aware of the paper [17]. Second-order conditions are usually very challenging for nonlinear systems due to the high technicality which is required for a rigorous treatment. As it happens frequently in the second-order analysis for systems of PDEs, we have to deal with the so-called two-norm discrepancy, as we can prove that the solution operator $\mathcal{S}$ (hence the reduced cost functional $\mathcal{J}_{\text {red }}$ ) is twice Fréchet differentiable with respect to one norm $\left(L^{\infty}(Q)^{2}\right)$, but the coercivity condition of the second derivative of $\mathcal{J}_{\text {red }}$ expressed by (6.10) holds just in a weaker norm $\left(L^{2}(Q)^{2}\right)$ in which the (second) derivative of the solution operator $\mathcal{S}$ (hence of $\mathcal{J}_{\text {red }}$ ) is not even well defined. To this concern, let us mention the work [4]; there, the authors show that, provided some additional properties are fulfilled, the two-norm discrepancy issue can be overcome by deriving the more natural sufficient condition in Theorem 6.1 with respect to $L^{2}-L^{2}$ norms instead of the $L^{2}-L^{\infty}$ situation. However, while ([4], Ass. (A1)) can easily be checked, the second assumption in this paper ([4], Ass. (A2)) requires a number of nontrivial properties to be proven (and we are not sure whether this can actually be done). For these reasons, we have chosen the present line of approach.

Acknowledgements. This research was supported by the Italian Ministry of Education, University and Research (MIUR): Dipartimenti di Eccellenza Program (2018-2022) - Dept. of Mathematics "F. Casorati", University of Pavia. In addition, PC and AS gratefully mention their affiliation to the GNAMPA (Gruppo Nazionale per l'Analisi Matematica, la Probabilità e le loro Applicazioni) of INdAM (Istituto Nazionale di Alta Matematica). Moreover, PC aims to point out his collaboration, as Research Associate, to the IMATI - C.N.R. Pavia, Italy. 


\section{REFERENCES}

[1] V. Barbu, Nonlinear Differential Equations of Monotone Type in Banach Spaces. Springer, London, New York (2010).

[2] H. Brezis, Opérateurs maximaux monotones et semi-groupes de contractions dans les espaces de Hilbert. Vol. 5 of NorthHolland Math. Stud.. North-Holland, Amsterdam (1973).

[3] H. Cartan, Calcul différentiel. Formes différentielles. Hermann, Paris (1967).

[4] E. Casas and F. Tröltzsch, Second order analysis for optimal control problems: improving results expected from abstract theory. SIAM J. Optim. 22 (2012) 261-279.

[5] C. Cavaterra, E. Rocca and H. Wu, Long-time dynamics and optimal control of a diffuse interface model for tumor growth. Appl. Math. Optim. 83 (2021) 739-787.

[6] P. Colli, M.H. Farshbaf-Shaker, G. Gilardi and J. Sprekels, Second-order analysis of a boundary control problem for the viscous Cahn-Hilliard equation with dynamic boundary condition. Ann. Acad. Rom. Sci. Ser. Math. Appl. 7 (2015) 41-66.

[7] P. Colli, G. Gilardi and D. Hilhorst, On a Cahn-Hilliard type phase field system related to tumor growth. Discret. Cont. Dyn. Syst. 35 (2015) 2423-2442.

[8] P. Colli, G. Gilardi, E. Rocca and J. Sprekels, Vanishing viscosities and error estimate for a Cahn-Hilliard type phase field system related to tumor growth. Nonlinear Anal. Real World Appl. 26 (2015) 93-108.

[9] P. Colli, G. Gilardi, E. Rocca and J. Sprekels, Asymptotic analyses and error estimates for a Cahn-Hilliard type phase field system modelling tumor growth. Discret. Contin. Dyn. Syst. Ser. S 10 (2017) 37-54.

[10] P. Colli, G. Gilardi, E. Rocca and J. Sprekels, Optimal distributed control of a diffuse interface model of tumor growth. Nonlinearity 30 (2017) 2518-2546.

[11] P. Colli, G. Gilardi and J. Sprekels, A distributed control problem for a fractional tumor growth model. Mathematics 7 (2019) 792.

[12] P. Colli, A. Signori and J. Sprekels, Optimal control of a phase field system modelling tumor growth with chemotaxis and singular potentials, Appl. Math. Optim. 83 (2021) 2017-2049.

[13] V. Cristini, X. Li, J.S. Lowengrub and S.M. Wise, Nonlinear simulations of solid tumor growth using a mixture model: invasion and branching. J. Math. Biol. 58 (2009) 723-763.

[14] V. Cristini and J. Lowengrub, Multiscale Modeling of Cancer: An Integrated Experimental and Mathematical Modeling Approach. Cambridge University Press (2010).

[15] M. Dai, E. Feireisl, E. Rocca, G. Schimperna and M.E. Schonbek, Analysis of a diffuse interface model of multi-species tumor growth. Nonlinearity 30 (2017) 1639-1658.

[16] J. Dieudonné, Foundations of Modern Analysis. Vol. 10 of Pure and Applied Mathematics. Academic Press, New York (1960).

[17] M. Ebenbeck and P. Knopf, Optimal control theory and advanced optimality conditions for a diffuse interface model of tumor growth, ESAIM: COCV 26 (2020) 1-38.

[18] M. Ebenbeck and P. Knopf, Optimal medication for tumors modeled by a Cahn-Hilliard-Brinkman equation. Calc. Var. Partial Differ. Equ. 58 (2019) 131.

[19] M. Ebenbeck and H. Garcke, Analysis of a Cahn-Hilliard-Brinkman model for tumour growth with chemotaxis. J. Differ. Equ. 266 (2019) 5998-6036.

[20] S. Frigeri, M. Grasselli and E. Rocca, On a diffuse interface model of tumor growth, Eur. J. Appl. Math. 26 (2015) $215-243$.

[21] S. Frigeri, K.F. Lam and E. Rocca, On a diffuse interface model for tumour growth with non-local interactions and degenerate mobilities, in Solvability, Regularity, and Optimal Control of Boundary Value Problems for PDEs, edited by P. Colli, A. Favini, E. Rocca, G. Schimperna, J. Sprekels. Vol. 22 of Springer INdAM Series. Springer, Cham (2017) 217-254.

[22] S. Frigeri, K.F. Lam, E. Rocca and G. Schimperna, On a multi-species Cahn-Hilliard-Darcy tumor growth model with singular potentials. Commun. Math Sci. 16 (2018) 821-856.

[23] S. Frigeri, K.F. Lam and A. Signori, Strong well-posedness and inverse identification problem of a non-local phase field tumor model with degenerate mobilities. Eur. J. Appl. Math. (2021) 1-42.

[24] H. Garcke and K.F. Lam, Well-posedness of a Cahn-Hilliard system modelling tumour growth with chemotaxis and active transport. Eur. J. Appl. Math. 28 (2017) 284-316.

[25] H. Garcke and K.F. Lam, Global weak solutions and asymptotic limits of a Cahn-Hilliard-Darcy system modelling tumour growth. AIMS Math. 1 (2016) 318-360.

[26] H. Garcke and K.F. Lam, Analysis of a Cahn-Hilliard system with non-zero Dirichlet conditions modeling tumor growth with chemotaxis, Discr. Contin. Dyn. Syst. 37 (2017) 4277-4308.

[27] H. Garcke and K.F. Lam, On a Cahn-Hilliard-Darcy system for tumour growth with solution dependent source terms, in Trends on Applications of Mathematics to Mechanics, edited by E. Rocca, U. Stefanelli, L. Truskinovski, A. Visintin. Vol. 27 of Springer INdAM Series. Springer, Cham (2018) 243-264.

[28] H. Garcke, K.F. Lam, R. Nürnberg and E. Sitka, A multiphase Cahn-Hilliard-Darcy model for tumour growth with necrosis. Math. Models Methods Appl. Sci. 28 (2018) 525-577.

[29] H. Garcke, K.F. Lam and E. Rocca, Optimal control of treatment time in a diffuse interface model of tumor growth. Appl. Math. Optim. 78 (2018) 495-544.

[30] H. Garcke, K. F. Lam, E. Sitka and V. Styles, A Cahn-Hilliard-Darcy model for tumour growth with chemotaxis and active transport. Math. Models Methods Appl. Sci. 26 (2016) 1095-1148.

[31] H. Garcke, K.F. Lam and A. Signori, On a phase field model of Cahn-Hilliard type for tumour growth with mechanical effects. Nonlinear Anal. Real World Appl. 57 (2021) 103192. 
[32] D. Gilbarg and N.S. Trudinger, Elliptic partial differential equations of second order, 2nd edn. Springer-Verlag, BerlinHeidelberg (1983).

[33] A. Hawkins-Daarud, K.G. van der Zee and J.T. Oden, Numerical simulation of a thermodynamically consistent four-species tumor growth model, Int. J. Numer. Math. Biomed. Eng. 28 (2011) 3-24.

[34] D. Hilhorst, J. Kampmann, T.N. Nguyen and K.G. van der Zee, Formal asymptotic limit of a diffuse-interface tumor-growth model. Math. Models Methods Appl. Sci. 25 (2015) 1011-1043.

[35] C. Kahle and K.F. Lam, Parameter identification via optimal control for a Cahn-Hilliard-chemotaxis system with a variable mobility. Appl. Math. Optim. 82 (2020) 63-104.

[36] O.A. Ladyženskaja, V.A. Solonnikov and N.N. Uralceva, Linear and Quasilinear Equations of Parabolic Type. Vol. 23 of Mathematical Monographs. American Mathematical Society, Providence, Rhode Island (1968).

[37] J.-L. Lions, Équations différentielles opérationnelles et problèmes aux limites. Die Grundlehren der mathematischen Wissenschaften, Bd. 111. Springer-Verlag, Berlin-Göttingen-Heidelberg (1961).

[38] L. Scarpa and A. Signori, On a class of non-local phase-field models for tumor growth with possibly singular potentials, chemotaxis, and active transport. Nonlinearity 34 (2021) 3199-3250.

[39] A. Signori, Optimal distributed control of an extended model of tumor growth with logarithmic potential. Appl. Math. Optim. 82 (2020) 517-549.

[40] A. Signori, Optimality conditions for an extended tumor growth model with double obstacle potential via deep quench approach. Evol. Equ. Control Theory 9 (2020) 193-217.

[41] A. Signori, Optimal treatment for a phase field system of Cahn-Hilliard type modeling tumor growth by asymptotic scheme. Math. Control Relat. Fields 10 (2020) 305-331.

[42] A. Signori, Vanishing parameter for an optimal control problem modeling tumor growth. Asymptot. Anal. 117 (2020) 43-66.

[43] A. Signori, Penalisation of long treatment time and optimal control of a tumour growth model of Cahn-Hilliard type with singular potential. Discrete Contin. Dyn. Syst. 41 (2021) 2519-2542.

[44] J. Simon, Compact sets in the space $L^{p}(0, T ; B)$. Ann. Mat. Pura Appl. 146 (1987) 65-96.

[45] J. Sprekels and F. Tröltzsch, Sparse optimal control of a phase field system with singular potentials arising in the modeling of tumor growth. ESAIM: COCV $\mathbf{2 7}$ (2021) 2.

[46] J. Sprekels and H. Wu, Optimal distributed control of a Cahn-Hilliard-Darcy system with mass sources. Appl. Math. Optim. 83 (2021) 489-530.

[47] F. Tröltzsch, Optimal Control of Partial Differential Equations: Theory, Methods and Applications. Vol. 112 of Graduate Studies in Mathematics. American Mathematical Society, Providence, Rhode Island (2010).

[48] S.M. Wise, J.S. Lowengrub, H.B. Frieboes and V. Cristini, Three-dimensional multispecies nonlinear tumor growth I: Model and numerical method. J. Theor. Biol. 253 (2008) 524-543. 